\title{
Propagation and Nonlinear Scattering of Ultrashort Pulses - Examples of Modeling and Applications
}




\section{Propagation AND Nonlinear SCATTERING OF ULTRASHORT Pulses - EXAMPles OF Modeling AND Applications}

Willem Paul Beeker 


\section{Samenstelling van de promotiecommissie:}

\section{Voorzitter \& secretaris:}

prof. dr. G. van der Steenhoven (University of Twente, The Netherlands)

\section{Promotor:}

prof. dr. K.-J. Boller (University of Twente, The Netherlands)

\section{Assistent-promotor:}

dr. C.J. Lee (University of Twente, The Netherlands)

\section{Leden:}

prof. dr. ing. D.H.A. Blank (University of Twente, The Netherlands)

prof. dr. C. Fallnich (Westfälische Wilhelms-Universität Münster, Germany)

prof. dr. J.L. Herek (University of Twente, The Netherlands)

dr. A.P. Mosk (University of Twente, The Netherlands)

The research presented in this thesis was carried out at the Laser Physics and Nonlinear Optics group, Department of Science and Technology, MESA ${ }^{+}$ Institute of Nanotechnology, University of Twente, P.O. Box 217, 7500 AE Enschede, The Netherlands, and was financially supported by the Dutch Ministry of Education, Culture and Science (OC\&W)

Copyright (C) 2010 by Willem P. Beeker

ISBN: 978-90-365-2965-5

Printed by Ipskamp Drukkers B.V., Enschede, The Netherlands 
Propagation and Nonlinear Scattering

of Ultrashort Pulses -

Examples of Modeling AND Applications

\section{Proefschrift}

ter verkrijging van

de graad van doctor aan de Universiteit Twente,

op gezag van de rector magnificus, prof. dr. H. Brinksma, volgens besluit van het College voor Promoties in het openbaar te verdedigen

op vrijdag 29 januari 2010 om 16.45 uur

door

Willem Paul Beeker

geboren op 15 mei 1980

te Zwolle 
Dit proefschrift is goedgekeurd door:

De promotor: $\quad$ prof. dr. K.-J. Boller

De assistent-promotor: dr. C.J. Lee 
Voor mijn

Opa's en Oma's 
$\oplus$ 


\section{Contents}

Publications $\quad$ xi

Samenvatting $\quad$ xiii

1 Introduction 1

2 Theory 11

2.1 Nonlinear optics . . . . . . . . . . . . . . . . . . . . . . 11

2.1.1 Nonlinear polarization . . . . . . . . . . . . . . . 11

2.1.2 Second Harmonic Generation . . . . . . . . . . . . . . 12

2.1.3 Crystal classes . . . . . . . . . . . . . . . . . 13

2.1.4 Tensor elements and polarization angle . . . . . . . . 13

2.2 Density matrix models . . . . . . . . . . . . . . . . . . . 15

2.2 .1 Two-level system . . . . . . . . . . . . . . . . . . . 17

2.2 .2 Rabi oscillations . . . . . . . . . . . . . 18

2.2 .3 Maxwell equations . . . . . . . . . . . . . . . . 20

$2.2 .4 \quad \Lambda$-type system $\ldots \ldots \ldots \ldots \ldots$

2.2 .5 Light field propagation . . . . . . . . . . . . . . . 22

3 Polarization dependent SHG for powder sizing 25

3.1 Motivation . . . . . . . . . . . . . . . 25

3.2 Introduction . . . . . . . . . . . . . . . . . . . 26

3.3 Experimental . . . . . . . . . . . . . . . . . . . . . . 28

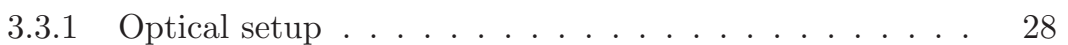

3.3 .2 Data analysis . . . . . . . . . . . . . . . . 29

3.3.3 Relationship between chord length and mean particle size 33

3.4 Results . . . . . . . . . . . . . . . . . . . . . . . . . 35

3.4.1 Single grain experiment . . . . . . . . . . . . . 36

3.4 .2 Multiple single grains . . . . . . . . . . . . . . 38

3.4.3 Front layer sensitivity in powders . . . . . . . . . . . . 39

3.4.4 Particle sizing of $\alpha$-lactose powders . . . . . . . . . . 41

3.4.5 Comparison of sizing methods . . . . . . . . . . . . . 41

3.5 Conclusions and outlook . . . . . . . . . . . . . . . . 42

vii

(1)

ii

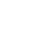

1

11
12
13
15
17
18
1
2


4 Numerical modeling of CARS $\quad \mathbf{4 5}$

4.1 Introduction . . . . . . . . . . . . . . . . . . . 45

4.2 Theoretical framework . . . . . . . . . . . . . . . . . . 49

4.2.1 Level scheme . . . . . . . . . . . . . . . . . . 49

4.2.2 Spectrum and emission peaks . . . . . . . . . . . . 49

4.2 .3 Numerical model . . . . . . . . . . . . . . . . . 52

4.2.4 Tests of the numerical model . . . . . . . . . . . 52

4.3 Enhancement of CARS emission efficiency . . . . . . . . . . 54

4.3.1 Stokes pulse delay ............... . . 56

4.3.2 Probe pulse delay . . . . . . . . . . . . . . 57

4.4 Conclusion ....................... 58

5 Sub-diffraction-limited resolution CARS 59

5.1 Overview of schemes for improvement of spatial resolution . . . 60

5.1.1 Reduced excitation area by nonlinear interaction . . . . 61

5.1 .2 Reduced excitation area by suppression . . . . . . . . 61

5.2 CARS emission suppression by interference . . . . . . . . . . 66

5.2.1 Concept and numerical model . . . . . . . . . . . 67

5.2 .2 Results ..................... 68

5.2 .3 Improvement of resolution . . . . . . . . . . . . 70

5.3 CARS emission suppression by population of vibrational level . 71

5.3.1 Concept and numerical model . . . . . . . . . . . 71

5.3 .2 Results ..................... 73

5.3 .3 Improvement of resolution . . . . . . . . . . . . 75

5.4 CARS emission modulation by Rabi oscillations . . . . . . . . . 76

5.4 Concept and numerical model . . . . . . . . . 76

5.4 .2 Results .................... 77

5.4 .3 Improvement of resolution . . . . . . . . . . . . . 79

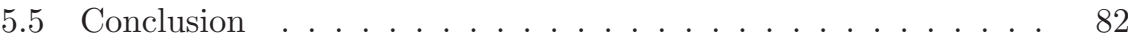

6 Numerical model of SIT solitons in $\Lambda$-type media 85

6.1 Introduction . . . . . . . . . . . . . . . 85

6.2 Numerical model . . . . . . . . . . . . . . . . . . . . 86

6.3 Test of the numerical model . . . . . . . . . . . . . . . 88

6.3.1 Soliton propagation .............. 89

6.3.2 Secant hyperbolic pulse shape . . . . . . . . . . . . 89

6.3.3 Area theorem . . . . . . . . . . . . . . . . 92

6.3.4 Colliding solitons . . . . . . . . . . . . . . . 92

6.3.5 Validation of the model . . . . . . . . . . . . . . 94

6.4 Capture and cloning event . . . . . . . . . . . . . . . . . . . 94

6.5 Phase relations . . . . . . . . . . . . . . . . . 96

6.6 Conclusion . . . . . . . . . . . . . . . . . . . . 98 
7 Nonlinear SIT soliton propagation in $\Lambda$-type media $\quad 99$

7.1 Motivation ... . . . . . . . . . . . . . . . . 99

7.2 Logical operations on solitons . . . . . . . . . . . . . . . . 101

7.2 .1 Initial SIT capturing event . . . . . . . . . . . . . . 101

7.2 .2 Second SIT trapping event . . . . . . . . . . . . . . . . 102

7.2 .3 Optical logic gates . . . . . . . . . . . . . . . . 106

7.2.4 Multiple trapping events . . . . . . . . . . . . . . . 106

$7.2 .5 \pi$-pulse operation . . . . . . . . . . . . . . . 108

7.3 Reading and clearing of stored ground state coherence . . . . . 109

7.3.1 Propagation as bi-solitons . . . . . . . . . . . . . . . 110

7.4 Conclusions . . . . . . . . . . . . . . . . . . . . . . 115

8 Conclusions and discussion 117

$\begin{array}{ll}\text { Bibliography } & 123\end{array}$

$\begin{array}{ll}\text { Dankwoord } & 133\end{array}$ 
$\bigoplus$ 


\section{Publications}

\section{Articles}

- W. P. Beeker, P. Groß, C. J. Lee, C. Cleff, H. L. Offerhaus, C. Fallnich, J. L. Herek and K. J. Boller, "A route to sub-diffraction-limited CARS Microscopy," Optics Express, 17, 25, 22632-22638, (2009).

- W. P. Beeker, P. Groß, C. J. Lee, C. Cleff, H. L. Offerhaus, C. Fallnich, J. L. Herek and K. J. Boller, "Spatially dependent Rabi oscillations: an approach to sub-diffraction-limited CARS microscopy," Physical Review A, (accepted for publication).

- W. P. Beeker, C. J. Lee, C. L. Strachan, P. Karmwar and K. J. Boller, "Measuring particle size of powders using polarization dependent SHG microscopy," (in preparation).

- W. P. Beeker, C. J. Lee, E. Can and K. J. Boller, "All-Optical Computing with Solitons Trapped in $\Lambda$-type Media," (in preparation).

\section{Oral presentations}

- W. P. Beeker, P. Groß, C. J. Lee, C. Cleff, H. L. Offerhaus, C. Fallnich, J. L. Herek and K. J. Boller, "Molecule-selective microscopy with subdiffraction-limited resolution - a theoretical investigation," Mesa+ annual meeting, Enschede, The Netherlands, (2009).

- W. P. Beeker, P. Groß, C. J. Lee, C. Cleff, H. L. Offerhaus, C. Fallnich, J. L. Herek and K. J. Boller, "Sub-Diffraction Resolution CARS Microscopy via Rabi Modulation: a Theoretical Investigation," The European Conference on Lasers and Electro-Optics, München, Germany, (2009).

- W. P. Beeker, P. Groß, C. J. Lee, C. Cleff, H. L. Offerhaus, C. Fallnich, J. L. Herek and K. J. Boller, "Sub-Diffraction Limited CARS Microscopy: A Theoretical Investigation," Conference on Laser and Electro-Optics / International Quantum Electronics Conference (CLEO/IQEC), Baltimore, MD, (2009).

- W. P. Beeker, C. J. Lee, C. J. Strachan and K. J. Boller, "Measuring Particle Size Distributions via the Polarization Dependence of Second 
Harmonic Generation," Conference on Laser and Electro-Optics / International Quantum Electronics Conference (CLEO/IQEC), Baltimore, MD, (2009).

- W. P. Beeker, C. J. Lee, L. Huisman and K. J. Boller, "Scattering and autocorrelation of ultra-short laser pulses in particulates," Physics@FOM Veldhoven 2008, Veldhoven, The Netherlands, (2008).

\section{Poster presentations}

- W. P. Beeker, E. Can, C. J. Lee and K. J. Boller, "Calculation of SIT Soliton Collisions for Optical Computing," The European Conference on Lasers and Electro-Optics, München, Germany, (2009).

- W. P. Beeker, C. J. Lee and K. J. Boller, "Powder characterization by scanning SHG intensity measurements," Thirteenth Annual Symposium of the IEEE/LEOS Benelux Chapter, Enschede, The Netherlands, (2008).

- W. P. Beeker, L. Huisman, C. J. Lee and K. J. Boller, "IFROG measurements on powder samples,"MESA+ annual meeting, Enschede, The Netherlands, (2008).

- W. P. Beeker, D. N. Deriga and K. J. Boller, "Capture of solitons in layered phaseonium," International Quantum Electronics Conference, Tokyo, Japan, (2005). 


\title{
Samenvatting
}

\author{
Niet lineaire verstrooiing en voortplanting van ultra korte pulsen - \\ voorbeelden van modellen en toepassingen
}

In dit proefschrift worden een aantal modellen en experimenten beschreven om de bruikbaarheid van een aantal specifieke vormen van niet-lineaire interactie tussen ultra korte licht pulsen en materie te bestuderen voor gebruik in een aantal specifieke toepassingen.

\section{Korrelgrootte metingen van droge poeders doormiddel van tweede- harmonische-generatie}

Alhoewel er vele methoden zijn om de korrelgrootte distributie in poeders te meten, zoals met behulp van microscopie, dynamisch licht verstrooiing en laser diffractie, zijn deze methoden behept met een aantal nadelen wanneer deze moeten worden toegepast in een industriële omgeving. In dit proefschrift wordt een nieuwe manier gedemonstreerd om de grootte van korrels in poeders te meten doormiddel van een polarizatiehoek afhankelijke meting van tweede-harmonisch licht terwijl de gefocusseerde laser bundel zijwaarts beweegt over het poeder. Door het gereflecteerde pomp licht en het polarizatiehoekafhankelijke tweede-harmonisch licht te vergelijken tussen metingen aan een enkele laag van korrels en een volledig poeder, wordt uit de polarizatiehoek afhankelijkheid geconcludeerd dat informatie over de korrelgrootte van het poeder kan worden verkregen, terwijl deze informatie niet wordt verkregen uit het gereflecteerde pomp licht. Dit toont aan dat de polarizatiehoek afhankelijke meting van tweede-harmonisch licht de mogelijkheid biedt om de korrelgrootte van droge poeders te karakteriseren door gebruik van een volledig optische meetopstelling. Tevens is aangetoond dat een onderscheid gemaakt kan worden in kristal type tussen twee verschillende poeders door naar de vorm van de functie die de polarizatie afhankelijkheid beschrijft te kijken. Dit kan mogelijkerwijs gebruikt worden om de mengverhouding van twee verschillende poeders te bepalen. De eenvoud van deze methode is interessant voor een continue en direkte meting bij productieprocessen in de voedsel en farmaceutische industrie. 


\section{CARS microscopie met een resolutie beter dan de diffractie limiet}

Spectroscopische technieken zoals "coherent Anti-Stokes Raman scattering" (CARS) kunnen gebruikt worden om microscopische afbeeldingen te maken die gericht zijn op de aanwezigheid van specifieke moleculen. Echter, bij gebruik van conventionele microscopen is de optische resolutie die behaald kan worden beperkt door de diffractie limiet. Deze limiet, zoals die reeds is geformuleerd door Abbe, beschrijft de fundamentele beperking in de resolutie van een microscoop opstelling. Er bestaan echter een aantal specifieke methoden om deze limiet te doorbreken. Tot nu toe zijn deze methoden nog niet direct toegepast op CARS aangezien er geen rechtstreekse methode is om deze te combineren. In dit proefschrift wordt een numeriek model gepresenteerd, gebaseerd op de dichtheids-matrix vergelijkingen om het CARS emissie proces te beschrijven, met als doel om CARS spectroscopie te combineren met technieken waarbij een hoge resolutie verkregen wordt. Daarbij wordt aangetoond dat dit model gebruikt kan worden om de dynamiek van de populatie dichtheden en coherenties van een drie-niveau systeem te bestuderen. Het emissie spectrum wordt verkregen doormiddel van het tijdsverloop van de coherenties. Enkele bekende eigenschappen van CARS emissie worden gemodelleerd om het model te verifiëren, hieruit blijkt dat het drie-niveau model voldoende gedetaileerd is om alle fysische fenomenen die worden geobserveerd in echte CARS experimenten te modelleren.

Door de Stokes puls eerder te laten arriveren dan de pomp puls en de probe puls te vertragen ten opzichte van deze pulsen wordt aangetoond dat een hogere efficiëntie om CARS emissie op te wekken wordt verkregen. Het model wordt gebruikt om, kwalitatief, de experimentele resultaten van Sautenkov et al. te bestuderen. In ons model wordt een maximale verhoging van de efficiëntie van $25 \%$ gevonden voor het geval van een gedegenereerde pomp en probe puls. Echter, het is de verwachting dat dit resultaat aan variatie onderhevig is afhankelijk van de levensduur en decoherentie-tijd van het materiaal. We tonen verder aan dat de efficiëntie verder verhoogd kan worden, wanneer de probe puls vertraagd aankomt ten opzichte van beide andere pulsen.

Een aantal routes om CARS microscopie met sub-diffractie gelimiteerde resolutie technieken te integreren worden beschouwd door de daarbij behorende dichtheids-matrix modellen te gebruiken. Hiermee is het interferentie proces zoals beschreven door Nikolaenko et al. bestudeerd en hun claim dat dit proces gebruikt kan worden om CARS emissie te onderdrukken is geverifieerd. Echter, er is geen mogelijkheid gevonden die hun suggestie realiseert dat dit onderdrukkingsproces gebruikt kan worden voor het verkrijgen van afbeeldingen met een resolutie kleiner dan de diffractie limiet.

Verder wordt, gebruik makend van het numerieke model, een manier gedemonstreerd waarmee CARS microscopie met een sub-diffractie-gelimiteerde resolutie verkregen kan worden op een manier die overeenkomsten vertoont met het proces van "stimulated emission depletion" (STED). We tonen aan dat een intense controle laser bundel gebruikt kan worden om CARS emissie te onderdrukken als de controle laser voortijdig de populatie van een aanvul- 
lende vibrationele toestand laat groeien, welke via een niet-stralend proces is gekoppeld aan de vibrationele toestand die wordt bemonsterd met het CARS proces. De berekeningen die uitgaan van typische waarden voor de moleculaire transities zoals die in CARS gebruikt worden, laten zien dat een onderdrukking tot $99.8 \%$ verkregen kunnen worden, wat overeenkomt met een resolutie van $\frac{\lambda}{22 \cdot \mathrm{NA}}$ (met $\lambda$ de golflengte van het licht en NA de numerieke apertuur van de microscoop).

Als alternatief, niet gebaseerd op een verzadigbare onderdrukkingsproces, maar gebaseerd op het locaal variëren van de amplitude modulatie veroorzaakt door Rabi oscillaties, demonstreren we een tweede route (in theorie) om CARS afbeeldingen te maken met een hoge resolutie. Door een energetische overgang met een relatief lange decoherentie-tijd in het molecuul resonant aan te drijven met een controle laser, worden zijbanden in het spectrum gecreeerd, die afhankelijk zijn van de locale intensiteit van de laser. Door deze Rabi splitsing van de CARS emissie accuraat te meten, kan de bron afgebeeld worden met een resolutie die wordt geschat op $65 \mathrm{~nm}$. Daarnaast zijn de Rabi zijbanden ook aanwezig bij andere emissie lijnen, waardoor de voorgestelde methodiek breder toepasbaar is.

\section{Soliton interacties voor volledig-optische berekeningen}

De sterke coherente interacties tussen media van het $\Lambda$-type en resonante licht pulsen is het onderwerp van veel research van de laatste tijd. De interesse hierin bestaat uit de mogelijkheid deze interactie te gebruiken voor bijvoorbeeld quantum geheugen systemen en quantum logische operaties en opslag van afbeeldingen. Het gebruik van "Self Induced Transparency" (SIT) solitonen lijkt een interessante kandidaat te zijn voor quantum berekeningen met gebruik van continue variabelen. De interactie, benodigd voor dergelijke toepassingen, wordt veelal gerealiseerd door een niet-resonant Kerr effect, deze vereist echter een lange interactielengte of een sterke vorm van interactie. SIT is een resonant effect met een sterke interactie tussen lichtpuls en medium, dit biedt de mogelijkheid om de interactielengte te verkorten.

Om de interactie tussen dergelijke SIT solitonen en het medium te modelleren, wordt een numeriek model gepresenteerd, dat is gebaseerd op een gekoppelde set van de dichtheids vergelijkingen en de golfvergelijking van Maxwell voor een $\Lambda$-type medium. De werking van dit model wordt geverifieerd aan de hand van enkele bekende eigenschappen van de voortplanting van SIT solitonen en de wisselwerking van deze solitonen met het medium onder verschillende omstandigheden, door deze te vergelijken met de resultaten uit de beschikbare literatuur en blijkt in overeenstemming te zijn. Met dit model wordt de vangst van een SIT soliton door een zwakke controle laser puls aangetoond. Door een dergelijk vangst proces wordt een intense puls gevormd die het medium verlaat en wordt een grondtoestands-coherentie gecreëerd die achterblijft in het medium. De relatie tussen de fases van de ingebrachte lichtpulsen, de ruimtelijke structuur van de achterblijvende grondtoestands-coherentie en 
pulsen die het medium verlaten wordt gepresenteerd.

Verder wordt een zeer niet-lineair type puls propagatie onderzocht waarbij een tweede SIT soliton botst met een eerder gevormde grondtoestands-coherentie structuur. De relatie tussen de fasen van de inkomende SIT solitonen en de zwakke controle laser puls en de uittredende pulsen wordt gepresenteerd, waarbij wordt aangetoond dat, onder bepaalde omstandigheden, deze relatie identiek is aan die welke de "exclusive OR-gate" beschrijft. Bij het verwijderen van de grondtoestands-coherentie met een intense controle laser blijkt dat een bi-soliton wordt gevormd die zich door het medium voortplant. 


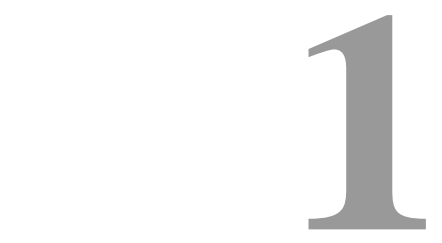

\section{Introduction}

Light sources capable of producing ultrashort pulses have become available by the invention of the laser [1] and, particularly, the technique of mode-locking (see, e.g., [2]). The advantage of pulsed lasers is that the average output power can be kept low, while the peak intensity can be very high. Such intense pulses, incident on a medium, perturb the state of the medium to such an extent that the nonlinear response of the material becomes both large and interesting. Such nonlinear responses form the basis of a wide range of applications and fundamental research.

To classify the large variety of nonlinear responses, the order of nonlinear interaction is often expressed by the number of light fields that drive the nonlinear response, e.g., a second order nonlinear response is caused by two light fields (which may be degenerate). Independent of the order of the response, there is a back-action on the driving electromagnetic fields. This back-action can be described via Maxwell's equations, where the response of the medium acts as a source term. Then, the physical manifestation of the nonlinear interaction can be divided roughly into whether the response occurs at one of the frequencies of the injected light, or at a different frequency. This means that a nonlinear interaction may appear either as an optically induced change of the medium's index of refraction, absorption, and amplification on the one hand, or that on the other hand the interaction appears to generate additional light frequencies, which is commonly called nonlinear optical frequency conversion.

An example for each of these two different types of nonlinear interactions and possible applications is optical parametric down-conversion and the optical Kerr effect. Such down-conversion can be used, for instance, in optical parametric oscillators (OPO's) [3], to produce coherent light with wavelengths which would otherwise be more difficult to provide with conventional laser tech- 
nology. The optical Kerr effect, which is the change in the effective refractive index of the material due to the intensity of the light propagating through the medium, can be used, for instance, to passively mode-lock a laser [4].

In this thesis, new proposals are formulated to exploit or modify the effects of second order and third order nonlinear interactions of ultrashort pulses of light with matter for various applications. More specifically, the polarization dependency of the second order nonlinear process of second harmonic generation (SHG) is used to measure the average grain size and distribution of sizes in powders. Furthermore, the light induced suppression and modification of third-order nonlinear optical emission from molecules is investigated theoretically. These studies have allowed us to propose two new, high resolution imaging techniques based on ultrashort pulses. Finally, in related work, the resonant nonlinear interaction between special ultrashort pulses, so-called solitons based on self induced transparency (SIT) are studied and we show that they can be used to form an all-optical logic gate for all-optical computing.

The three main nonlinear interaction regimes, categorized by the nonlinear excitation process, used in this thesis, are summarized in the schematic representation as seen in fig. 1.1. Although these three types of interaction and the numerical modeling thereof are usually presented as different physical processes. At close inspection, however it shows that these interactions bear mutual resemblances which is a common energy level scheme comprising three levels and in which only the number of injected light frequencies and heir detunings from resonances is varied. These resemblances can be exploited by making use of the similarities of the numerical models describing these processes, thereby enabling to look upon what is beyond the standard description via separate approaches.

In this comparison, SHG (fig. 1.1 (left)) can actually be seen as a rather simple nonlinear process because there is only a single driving light frequency which is usually very far detuned from single and two-photon resonances. Thus, SHG can usually be described as a small perturbation of the medium seen as a classical, driven oscillator, where a small and constant coefficient, here $\chi^{(2)}$, quantifies the weak nonlinear response. If the interaction lengths are short, another simplification in the description of SHG is that the integration of Maxwell's equations can be restricted to yield a small change of the field which remains proportional to the $\chi^{(2)}$ coefficient. The relative simplicity of SHG obtained with large detunings and small interaction lengths certainly does not leave much room in discovering novel features in the physical process itself. However, the simple relation between input and output obtained thereby is also the strength of the process when investigating novel applications in an experimental approach. Indeed, in this thesis we have employed the relative simplicity of SHG to experimentally demonstrate a novel application of SHG in the optical characterization of grain size in powders.

The second process described in fig. 1.1 (center) that we investigated is Coherent anti-Stokes Raman scattering (CARS). This process is commonly seen as a nonlinear conversion process, similar to SHG only with more light frequen- 


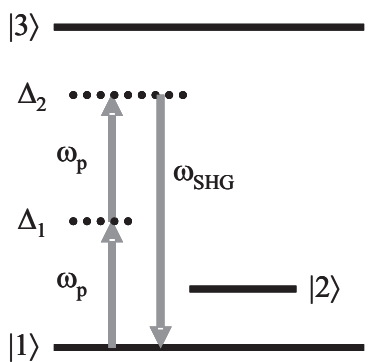

SHG

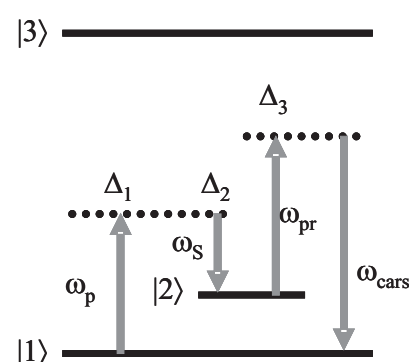

CARS

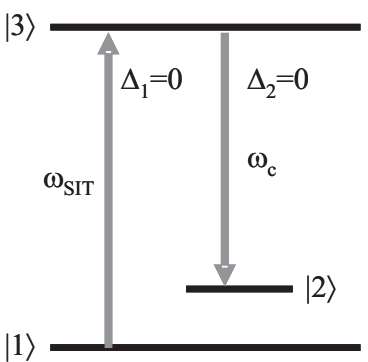

soliton

Figure 1.1: Schematic overview of the three types of nonlinear optical pulse propagation that we have investigated, with their main difference being only the number and detunings of the injected light fields: SHG (left), CARS (center) and SIT solitons (right). For SHG only one pump field is injected $\left(\omega_{p}\right)$. The field is far detuned from the excited state $\left(\Delta_{1}\right)$, even for the case of two-photon excitation $\left(\Delta_{2}\right)$, and induces only a weak nonlinear polarization in the medium, which radiates light $\left(\omega_{S H G}\right)$ at twice the pump frequency. For CARS, three light fields are injected and chosen as partly resonant. The pump $\left(\omega_{p}\right)$ and Stokes $\left(\omega_{S}\right)$ are two-photon resonant $\left(\Delta_{1}=\Delta_{2}\right)$ with the $|1\rangle-|2\rangle$ transition, which induces a resonant polarization of the medium. The probe field $\left(\omega_{p r}\right)$ is chosen off-resonant and is scattered nonlinearly due to this polarization such that a CARS light field $\left(\omega_{\text {cars }}\right)$ is emitted. For SIT solitons, two light fields are injected and both are chosen as fully resonant. The SIT light field $\left(\omega_{S I T}\right)$ is resonant with the $|1\rangle$ $|3\rangle$ transition, in addition, the control light field $\left(\omega_{c}\right)$ is resonant with the $|2\rangle-|3\rangle$ transition, causing a strong interaction between the medium and the light fields. 
cies involved that are all largely detuned from resonance. However, this view is not fully adequate because there is also a weak resonant excitation involved, i.e., two-photon resonance with the $|1\rangle-|2\rangle$ transition. This resonance requires that the medium is described in more detail than just via a nonlinear coefficient, particularly with respect to the excitation of population across the $|1\rangle-|2\rangle$ transition. This can be achieved through a quantum mechanical description of the medium via the density matrix, and if the interaction lengths can be kept short, such as in microscopic applications of CARS, the integration of Maxwell's equations can still be simplified or omitted. In this thesis we show that the detailed description of CARS using the density matrix approach, thereby tracing also population changes, is a key to look upon potential improvements of CARS microscopy towards sub-diffraction limited spatial resolution.

The third scheme in fig. 1.1 (right) describes the most complex situation that can be encountered for light propagation in three-level media in that here the injected light fields are fully resonant with all transitions, thereby generating the maximum nonlinear response of the medium. Also, to allow for maximum nonlinear interaction by maximum back-action the approximation of a short interaction length is given up and instead the medium is given a high optical density such that, without the nonlinearity in pulse propagation dynamics the medium would be fully opaque. For this situation we have discovered and explored highly interesting pulse propagation dynamics based on so-called self-induced transparency and soliton pulses that involved the storage of the phase of light pulses and the mutual all-optical control of light pulses with each other. To describe such pulse propagation dynamics it is required to track all populations on all levels using the density matrix equations and simultaneously, integrate Maxwell's equations. These studies, due to the high complexity of the underlying pulse propagation dynamics is currently far from a direct experimental demonstration, but it is also the high degree of nonlinearity and complexity which enables us to look into largely undiscovered ranges in nonlinear pulse propagation.

In the remaining part of the introduction we will further detail the importance and applications that are related to the studied processes of SHG, CARS and SIT soliton propagation.

\section{SHG-based particle sizing technique of dry powders}

Although there are many methods to measure the size distribution of powders, such as microscopy $[5,6]$, dynamic light scattering $[6,7]$, and laser diffraction [8], these existing methods suffer from numerous disadvantages when applied in industry during processing (such as milling, granulation, mixing and compaction).

For instance, microscopy, dynamic light scattering and laser diffraction all require that the materials in the production process are sampled and, in the case of dynamic light scattering the sample must be diluted such that it is safe to assume that each detected photon has only scattered once [7]. Sim- 
ilarly, laser diffraction relies on analyzing the diffraction off single particles, and, therefore, requires substantial dilution [8]. Although both of these technologies produce a particle size distribution by measuring only one particle at a time, they can provide high throughputs, for instance, a typical commercially available particle sizer using laser diffraction measures a few grams of sample in approximately a minute. However, the dilution requirement precludes their use in many industrially relevant situations, e.g., during tableting. Microscopy, on the other hand is much slower, although it offers the benefit of measuring the sizes of different particles in parallel. Depending on the software analysis technique used, it does not necessarily require substantial dilution. For example, in the case of neural network, shadow analysis techniques, no dilution is required, but the accuracy of the resulting particle size distribution depends strongly on the training set [9]. Edge analysis is a common technique [10], but the accuracy of the results depends on the sharpness of the edges, and some dilution may be necessary so that particles are not overlaying one another in the field of view. The biggest disadvantage of microscopy is the high precision focusing and sampling mechatronics required to obtain sufficiently high sample throughput. This makes the instrument expensive and potentially delicate, and, therefore, not suited to an industrial environment.

In addition to different implementations of particle sizing, due to their different working principle, the different techniques report different particle size distributions even with the same sample. Dynamic light scattering measures the photon counting statistics at an angle $\theta$ to the laser beam angle of incidence. The time between photon arrival times is related to the motion of the particle, which, in turn, is related to the dynamic viscosity of the fluid and the so-called hydrodynamic radius of the particle. In irregularly shaped particles, the hydrodynamic radius often corresponds most closely to the longest distance between the edges of the particle. Laser diffraction, in contrast, analyzes the spacing between diffraction maxima, which depends on the volume of the particle, and, as a result, the particle size distribution corresponds to the distribution of spherical radii that corresponds to the measured volume distribution. These two examples highlight the difficulty in comparing particle size distributions reported by different techniques and even defining the particle size distribution.

As an alternative measurement technique we present an experimental realization of an optical setup based on analyzing the coherent nonlinear optical response of a powder to excitation with short laser pulses in chapter 3. More specifically, we show how the polarization dependence of SHG from powder samples can be used for particle sizing. This technique can, in principle, be used in-line in an industrial process. By scanning a focused laser spot across the surface of a powder, we obtain a set of chord lengths, which is the path of the light across each scanned single particle. The average of these chord lengths is related to the mean particle size as will be shown by a numerical model. We also show that, after analysis of the measurement data, the average grain size and the particle size distribution can be determined, though this re- 
quires assuming that the particle can be loosely approximated by an ellipsoid. Furthermore, we show that a distinction with respect to the crystal class of the material can be made between two powders of different chemical composition using the same type of measurements.

\section{CARS microscopy beyond the diffraction limit}

For a long time it was thought that the spatial resolution $d$ of an image produced by an optical imaging system was limited by a fundamental limit in optics. This limit, known as the diffraction limit, as first approximated by Abbe [11], states that $d=\frac{\lambda}{2 \sin \alpha}$, with $\alpha$ the collection angle of the imaging system and $\lambda$ the wavelength of the detected light. As an example, for the field of microscopy, of the maximal attainable resolution due to the diffraction limit: a typical objective lens with a numerical aperture (NA) of 0.65 and a light source with a wavelength of $650 \mathrm{~nm}$ has a maximum resolution of $500 \mathrm{~nm}$.

Nevertheless, optical microscopy is the workhorse of biology, providing high contrast images, often in real time, of biological processes at sub-cellular distance scales. Ultimately, it is desirable to observe interactions at the single molecule or even single functional group level. This requires resolution in the nanometer range, which is well beyond the resolution of standard confocal microscopy. The diffraction limit, as formulated by Abbe in 1886 [11], describes the fundamental limit of resolution of optical systems, as were known at that time. Recent advances in the field of optics show that there are various methods to circumvent this theoretical limit, and go beyond it, such as stimulated emission depletion (STED) microscopy $[12,13]$, photo activated localization microscopy (PALM) [14], and stochastic optical reconstruction microscopy (STORM) $[15,16]$.

STED microscopy is based on the suppression of spontaneous emission from excited fluorophores by inducing a competing process of stimulated emission using an additional laser. Using, for example, a so-called doughnut shaped beam, the spontaneous emission is suppressed except for a small volume around the node of the beam. By attaching the fluorophores to specific functional groups of molecules, the position of those molecules can be resolved with a very high resolution. Recent results show that STED can provide images with a lateral resolution of $6 \mathrm{~nm}$ in solids [17] or $33 \mathrm{~nm}$ in liquids [18].

PALM (or STORM) microscopy is based on a set of fluorescence measurements. The fluorophores, attached to specific functional groups of molecules, are activated by a short laser pulse. However, this pulse has a small intensity and duration such that only a small fraction of the fluorophores is activated each time. By fitting the point spread function of the imaging system on each imaged molecule, the position of this molecule is determined with an accuracy to within the uncertainty of the fit. By repeating this excitation and measurement process several times, eventually all fluorophores have been excited, and the total image can be constructed with a resolution of the order of tens of nanometers. STORM therefore requires an extended time to construct an 
image. Furthermore, STORM requires rather specialized fluorescent labels [19].

A disadvantage of fluorescence microscopy techniques is that labels have to be introduced to the cell. The labels may influence metabolic processes in the cell, or, perhaps, lead to cytotoxicity $[20,21]$. The time period over which the cell can be imaged is limited because fluorescent labels suffer from photobleaching [22]. Furthermore, they may not attach to the correct molecule or functional group of interest [23]. Even if, ultimately, the exact position of the attached fluorophore is obtained by using sub-diffraction-limited imaging techniques, the obtained image has only imaged the fluorophore and not the cell, molecule or functional group of interest to which it had been attached. Thus, at very high resolutions (higher than currently available in liquid samples with STED, for instance), the labels themselves will become the limiting factor that determines the resolution. There is, therefore, great interest in developing new label-free imaging techniques which directly image the functional groups of interest.

Near field optical techniques such as scanning near-field optical microscopy (SNOM or NSOM) [24], are based on the measurement of the evanescent fields from objects and have been shown to provide sub-diffraction-limited resolution images with typical resolutions of 50-100 nm [25]. The evanescent fields contain high spatial frequencies, which, unlike the low spatial frequencies, do not propagate into the far field regime. By measuring these high spatial frequencies, images can be constructed with a very high spatial resolution, also of the order of tens of nanometers. However, the amplitude of the evanescent fields decays exponentially as a function of distance. Therefore, these techniques require an optical aperture, usually in the form of a tapered fiber, be placed within a few tens of nanometers of the region of interest, which typically limits the technique to surface mapping.

By probing samples with spectroscopic techniques such as Raman spectroscopy or Coherent Anti-Stokes Raman Scattering (CARS) spectroscopy, the specific vibrational transitions of molecules are addressed and vibrational coherences are excited, with which the chemical composition of the sample can be determined. By combining such spectroscopic techniques with microscopic techniques, images of samples are formed where the physical location of various spectroscopic features are mapped. By using spectroscopic features that are unique to the compounds of interest, an image that details the spatially dependent concentration of the compounds can be produced. Although there is a great interest in obtaining such images, so far, these spectroscopic techniques have not been combined with sub-diffraction-limited imaging techniques, since there is no straight-forward method to combine the underlying physical processes of both techniques.

In CARS two lasers are tuned such that a vibrational coherence is induced. This vibrational coherence induces frequency sidebands on a probe laser, which are measured. Since CARS is not based on emission processes from an excited level, a STED-like suppression process cannot be directly applied to CARS. Furthermore, since the formation of vibrational coherence is not a stochastic 
process, an image reconstruction technique such as in PALM cannot be applied to CARS either.

In this thesis we present two novel routes to combine the spectroscopic technique of CARS and its molecular specificity with a sub-diffraction-limited imaging method by modeling the optical response of a molecular sample using a quantum mechanical approach based on the so-called density matrix equations. The results, based on numerical evaluation of the model, presented in this thesis, show that a spatial resolution better than the diffraction limit can be obtained. These techniques make use of an additional laser that influences the standard CARS emission process by exciting an additional vibrational state in the molecule. We will show that, depending on the strength of the damping experienced by the molecular system, two related techniques can be employed to obtain sub-diffraction limited resolution imaging. When the damping is very strong, we show that the CARS emission can be suppressed by preventing the build-up of the required coherence. On the other hand, when damping is weak such that a significant vibrational coherence is built up by the additional laser, the coherence can be exploited to introduce spatially dependent sidebands on the CARS emission frequency. These sidebands can be used to construct an image with sub-diffraction limited resolution. A short introduction to the density matrix formalism is given in section 2.2 . The introduction to the modelspecific details and the validation of the validation is described in chapter 4 . Subsequently, the characteristics and expected performance of the two subdiffraction-limited resolution schemes are presented in chapter 5 .

\section{SIT soliton interactions for all-optical computing}

Strong and coherent interactions between $\Lambda$-type three-level atomic media and resonant light pulses have been a topic of much interest recently [26-28]. This interest has been driven by quantum memory systems $[29,30]$, quantum logic operations [31], and image storage [32]. In the case of quantum information processing, where it is necessary to store and process multiple quantum bits (qubits) in the same medium $[33,34]$, it is of great interest to examine the influence of multiple light pulses on the spatial distribution of the ground-state coherence of the medium.

In view of the latter, the use of solitons based on self induced transparency (SIT) [35] appears to be an interesting candidate for continuous variable quantum computing [36]. Continuous variable optical computing (quantum or classical) requires a nonlinear optical interaction [36]. Generally, the non-resonant Kerr effect is chosen as the nonlinear optical interaction [37,38], which unfortunately requires intense optical pulses or long interaction lengths $[39,40]$. SIT is a resonant effect [35], thereby providing a much stronger interaction. This offers the possibility of reducing the interaction lengths and pulse intensities required for continuous variable optical computing.

A further disadvantage of using the nonresonant Kerr effect is that the information is exchanged and stored between optical pulses that propagate with 
the speed of light. Therefore, operations between different pulses must be carefully timed so that the overlap between them in the nonlinear optical medium is maximized [40]. However, as we will show here, by using SIT solitons, the timing problem is much less critical. The information contained in SIT solitons can be stored in the ground state coherence of a three-level medium which does not propagate (is stationary) by applying an appropriate control light field [41,42]. New results, presented in chapter 7 , show that such SIT solitons allow for computational operations to be performed on the stationary coherence, rather than on the traveling soliton.

Through calculations of the coupled Maxwell and density matrix equations, we show that information contained in these soliton pulses can be stored within a $\Lambda$-type medium. Such an information storage process might be useful in all-optical computing where the information is carried by light pulses. The modeling of such soliton storage is described in chapter 6 .

Besides information storage, logic gates need to be implemented for alloptical computing. An example of such a gate, an exclusive OR-gate (XOR gate), is demonstrated by using the nonlinear interaction of two solitons within the three-level medium. Furthermore, it will be shown that these pulses create certain excited regions within the medium. These excited regions can be moved through the medium in a process in which bi-soliton pulses are formed. In chapter 7 the key features of such nonlinear interaction and propagation of optical solitons are presented.

This thesis is divided between theoretical modeling of atomic and molecular systems, and experimental work. Chapter 2 introduces the key concepts of the density matrix formalism that are then used to illustrate how to create the populations and coherences required to achieve sub-diffraction-limited resolution CARS images. The spatially selective suppression of CARS emission and the selective amplitude modulation of CARS emission are described in chapter 5 . As a next step, the density matrix model is extended by an improved description of light emission, absorption and conversion, to be able to model nonlinear pulse propagation also in resonant media with high optical densities. With this so-called Maxwell-Bloch model, we recall some properties of SIT solitons, and, in chapter 7 we show how all-optical logic gates can be implemented. Finally, some conclusions and proposals for future work are presented in chapter 8 . 
$\oplus$ 


\section{2 \\ Theory}

\subsection{Nonlinear optics}

\subsubsection{Nonlinear polarization}

The field of nonlinear optics describes the nonlinear optical response of a material or medium to the presence of a light wave. At low light intensities, such as provided by incoherent light sources, the response of the medium is predominantly a linear function of the incident field and shows, e.g., as the well known effects of absorption or refractive index. At high intensities, however, such as have become available through lasers that produce ultrashort pulses, the response of the medium becomes nonlinear and thus can lead to a wealth of complex, interesting and novel effects, or to potential novel applications. To describe the linear and nonlinear response of a medium in a most simple manner, the induced polarization $P(\omega)$ of the medium may be approximated by a Taylor expansion in $E$ [43]:

$$
P(\omega)=\chi^{(1)}(\omega) E+\chi^{(2)}(\omega) E^{2}+\chi^{(3)}(\omega) E^{3} \ldots
$$

Although this equation is written in a scalar form for brevity, $\chi^{(1)}(\omega)$ is actually a tensor which describes the linear optical properties (absorption and refraction) of the medium and how these vary as a function of the size of the electric field vector and its orientation relative to that of the medium. The higher order terms depend on higher order tensors $\chi^{(n)}$ which contain the nonlinear optical properties. In this Taylor approximation the magnitude of the nonlinear susceptibility tensors $\chi$ decreases quickly with increasing order $n$, such that the expansion converges for fields that are not too high. Furthermore, 
one usually considers that only a certain single higher order of the susceptibility dominates the response via a proper choice of the medium and the light intensity. To more detail, the response usually consists of the displacement of charged particles in the material, such as electrons following the acceleration induced by the rapidly oscillating electric field of an incident light wave. In most situations the induced charge displacement can be approximated as an oscillating volume density of the induced dipole moment called the induced polarization. This response of the medium becomes measurable via the additional electro-magnetic field that is radiated off by the induced polarization.

In section 2.1.4 the tensor properties of $\chi^{(2)}$ will be discussed in more detail. A well known example of a nonlinear response of a material is frequency doubling, also called Second Harmonic Generation (SHG), in this nonlinear optical process the medium radiates light at twice the frequency of the incident light. Other examples are the optical Kerr effect where the refractive index scales with the intensity of the light field, the electro optic effect where the refractive index scales with a static electric field and more general effects, such as the so-called coherent anti-Stokes Raman scattering (CARS) where the frequency of the radiated light is the sum and difference of three different incident light fields. In the following we begin with a short description of the simplest nonlinear process, SHG, to more detail to prepare the background for chapter 3 where an SHG-based measurement technique is presented to determine the mean particle size of a powder.

\subsubsection{Second Harmonic Generation}

SHG is a nonlinear process of second order, i.e., it can be described well by only the $\chi^{(2)}$-tensor being nonzero. A $\chi^{(2)}$-nonlinearity is found in media only if there is an asymmetry of the restoring force of electrons when displaced by an incident light field. Such asymmetry is provided by certain crystal classes as will be expanded upon in section 2.1.3.

In the inhomogeneous wave equation for the electro-magnetic field, that can be derived from the Maxwell equations [44], the temporally changing polarization of the medium acts as a source term, i.e., it is responsible for the generation of a light field. Due to the linearity of the Maxwell equations, this light field has the same frequency components as the polarization of the medium [44]. Even if the driving light field $E$ would contain only a single frequency (perfectly sinusoidal) component, the asymmetry of the restoring force, such as is the case in a non-centrosymmetric crystal, causes the polarization to oscillate in a fashion that deviates somewhat from a simple sinusoidal motion, such as with a smaller excursion to one side. Such a non-sinusoidal shape can be described by a Fourier expansion containing additional harmonics of the driving frequency, of which the second harmonic is the first to dominate when increasing the intensity. Thus, materials in which such an asymmetric restoring force occurs respond by radiating light which contains a component with twice the frequency of the pump light. The $\chi^{(2)}$ coefficient describing the 
strength of the SHG is usually very small compared to the $\chi^{(1)}$ coefficient for the linear response. On the other hand, the second term on the right hand side of eq. 2.1 grows with the square of $E$, this term can increase with regard to the first term via increasing $E$, to obtain, beyond a certain intensity, a noticeable SHG response. The intensity of the SHG light is related to the pump intensity as [43]:

$$
I_{S H G} \propto \chi^{(2)} I_{\text {pump }}^{2}
$$

The use of very intense pulses, such as formed by femtosecond lasers, increases the conversion efficiency of incident (pump) light to SHG light. Therefore, the use of such ultrashort pulses is of particular importance, when the goal is to make use of SHG in an application.

\subsubsection{Crystal classes}

The asymmetric restoring force, which is a prerequisite for the SHG process to occur, is found in so-called non-centrosymmetric crystals. While the structure of a centrosymmetric crystal has mirror planes across the main axes of the crystal, a non-centrosymmetric crystal has at least one plane across the main axis without mirror symmetry. Based on the symmetry properties in the arrangement of atoms within a crystal, all possible types of crystals can be classified in one of the 32 so-called point groups [43,45], of which 21 are noncentrosymmetric. Crystals from different point groups may show differences in the optical response. For instance, $\alpha$-lactose is monoclinic (crystal class point group 2) [46] which has a single nonlinear axis, whereas quartz is trigonal (crystal class point group 32) [43] and has two nonlinear axes which are oriented perpendicular with respect to each other (see also fig. 2.1).

\subsubsection{Tensor elements and polarization angle}

The effective scalar value of $\chi$ depends on the orientation of the angle of polarization of the pump light with respect to the axes of the nonlinear crystal. For instance, by varying the angle of polarization of the incident light field, the projection of this field on the optical nonlinear axis of the crystal is varied. For the case of crystals with only one nonlinear component along a single axis such as $\alpha$-lactose, this effective component of the electric field along the nonlinear axis induces a nonlinear polarization $P^{(2)}$ in the crystal only along a single axis,

$$
P^{(2)} \propto \chi^{(2)} E^{2} \cos ^{2}(\phi-\alpha)
$$

with $\chi^{(2)}$ being the optical nonlinear tensor coefficient of the crystal projected on the plane perpendicular to the incident beam, $E$ the electric field 


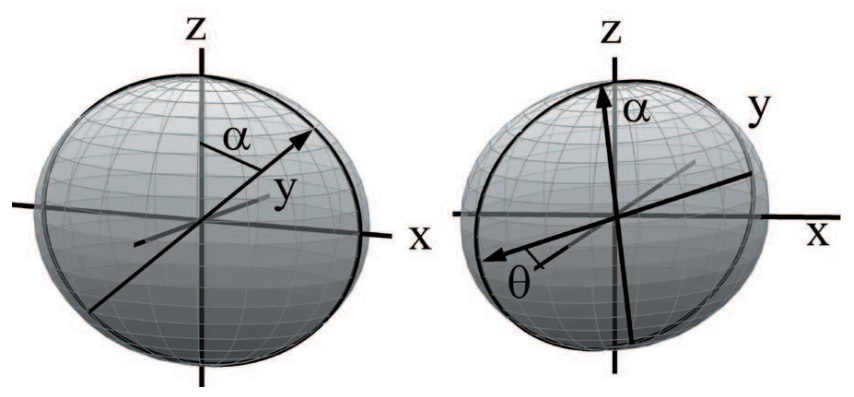

Figure 2.1: Schematic representation of the orientation of the nonlinear axes in a crystal with respect to the reference frame where $y$ denotes the direction of propagation of the light. The $x-z$ plane is the field of view onto which the nonlinear axes are projected. For the case of a single nonlinear axis (left) the angle $\alpha$ specifies the rotation of the nonlinear axis with respect to the $z$-axis. For the case of two nonlinear axes which are oriented perpendicular to each other, the projection of the axes is specified by two angles $\alpha$ and $\theta$.

strength of the incident light field and $\phi-\alpha$ the angle between the polarization angle of the light field and the optical nonlinear axis of the crystal. In our experiments, described in chapter 3, the intensity $I$ of this SHG light field is measured as a function of the polarization angle of the light field $\phi$ and, for the case of $\alpha$-lactose, is expected to be of the form of

$$
I(\phi)=A \cos ^{4}(\phi-\alpha)+C
$$

with $A$ a scaling factor and $C$ a constant background signal. For the case of quartz which has two nonlinear axes oriented perpendicular with respect to each other, the function is expected to be of the form of

$$
I(\phi)=C+A\left(\sin ^{2}(\theta) \sin ^{2}(\phi-\alpha)-\cos ^{2}(\phi-\alpha)\right)^{2}
$$

with $\alpha$ and $\theta$ the angles of the optical nonlinear axes of the crystal (as in fig. 2.1). The importance of these relations (eq. 2.4 and 2.5) is that they provide information on the nonlinear crystal class the sample consists of, which is very useful for analytical purposes in applications.

In the experiments described in chapter 3 the experimental measurement setup records the SHG intensity as a function of polarization angle and scanning position in order to measure chord lengths across particles in a powder. It will be shown that the average chord length corresponds to the mean particle size of the powder. Furthermore, it will be shown that the different polarization dependent responses of $\alpha$-lactose and quartz can be used to experimentally discriminate between powders of these two species. 


\subsection{Density matrix models}

In the previous sections, we described how the nonlinear response of a medium can be approximated to its most simple form via a Taylor expansion of the induced polarization, and how this description can form the basis of nonlinear pulse propagation aiming at an application. The observation that this approach works although the medium's nonlinear response is simply given by a coefficient $\left(\chi^{(2)}\right)$, is actually based on the circumstance that the involved light frequencies are far detuned from resonances, such as was indicated in chapter 1.

However, when the incident or generated fields are closer to resonances, the simplified approach fails and it becomes necessary to describe the response of the irradiated medium with more detail in a quantum mechanical picture. The advantage is then that not only the induced polarization but also population changes can be investigated, because, as will be seen, the distribution of population across the involved energy states has a great influence on the build-up of the medium's induced polarization. In particular, as will be described in chapters 4 and 5, the quantum mechanical modeling allows to devise ways to manipulate the dynamics of partly resonant or fully resonant nonlinear optical processes such as for achieving a higher spatial resolution in microscopic techniques.

In quantum mechanics, the measurable properties of a system made of small particles, such as atoms or molecules, are described via the wave function of the system. However, in most real systems, such as gases, liquids, and solids, the wave function is not known. Furthermore, even if the wave function of each atom in the system were known, the enormously high number of atoms typically involved would make it an impossible task to calculate the interactions with the different molecules and atoms. However, in such cases, one is often only interested in the ensemble properties. By limiting ourselves to ensemble averages, the problem of finding the properties of the system can be reformulated in terms of a density matrix. For a basic demonstration of the medium's response using the density matrix we recall the simplest case of a medium which consists of particles that can be approximated by a two-level system (Eigenstates $|1\rangle$ and $|2\rangle$ see fig. 2.2 (left)), and which is subject to a monochromatic laser light field of frequency $\omega$ [44].

The Schrödinger equation for such a two-level system describes its temporal development and is

$$
\begin{aligned}
& i \hbar \dot{a}_{1}(t)=E_{1} a_{1}(t)+V_{12} a_{2}(t) \\
& i \hbar \dot{a}_{2}(t)=E_{2} a_{2}(t)+V_{21} a_{1}(t)
\end{aligned}
$$

where $E_{1}$ and $E_{2}$ are the energies of the two Eigenstates and $V_{i j}$ is the matrix element of the potential energy associated with the external driving force (here the light field) and the $a$ 's are the probability amplitudes of the wavefunction $\Psi(t)=a_{1}(t)|1\rangle+a_{2}(t)|2\rangle$. The probability to find the system in the ground state, for instance, is $\left|a_{1}\right|^{2}$ whereas the sum over of probabilities over all states 
is normalized to unity:

$$
\left|a_{1}\right|^{2}+\left|a_{2}\right|^{2}=1
$$

The potential energy associated with the driving force can be expressed more explicitly as:

$$
\begin{aligned}
& V_{12}(t)=-e r_{12} \cdot \frac{1}{2}\left(\hat{\epsilon} E e^{-i \omega t}+\text { c.c. }\right) \\
& V_{21}(t)=-e r_{21} \cdot \frac{1}{2}\left(\hat{\epsilon} E e^{-i \omega t}+\text { c.c. }\right)
\end{aligned}
$$

where $e$ is the electron charge, $r_{12}=\langle 2|r| 1\rangle$ is the maximum electron displacement expectation value, $\hat{\epsilon}$ the polarization unit vector of the light field, $E$ the amplitude of the light field, and $\omega$ the frequency of the light field. In our modeling of nonlinear processes and pulse propagation, we generally have chosen the temporal profile of the light field envelopes $E(t)=E_{0} e^{-\frac{\left(t-\tau_{0}\right)^{2}}{\tau^{2}}} e^{-i t \Delta}$ as Gaussian functions each containing four parameters, the $1 / e^{2}$ width $\tau$, the peak amplitude $E_{0}$, the arrival time of the center of the pulse $\tau_{0}$ and the detuning $\Delta$ of the light filed carrier frequency $\omega$ from the two-level transition frequency $\omega_{21}, \Delta=\omega-\omega_{21}$. The transition frequency is give as

$$
\omega_{21}=\frac{E_{2}-E_{1}}{\hbar}
$$

We further introduce the standard expression for the field envelope $E$ to express the so-called Rabi frequency $\chi$

$$
\begin{aligned}
& \chi_{21}=e\left(r_{21} \cdot \hat{\epsilon}\right) \frac{E}{\hbar} \\
& \chi_{12}=e\left(r_{12} \cdot \hat{\epsilon}\right) \frac{E}{\hbar}
\end{aligned}
$$

where $\chi$ is the field-atom interaction energy in frequency units and $\hbar$ the reduced Planck constant. The set of differential equations (eq. 2.6 and 2.7 can be transformed using trial solutions of the form:

$$
\begin{aligned}
& a_{1}(t)=c_{1}(t) \\
& a_{2}(t)=c_{2}(t) e^{-i \omega t}
\end{aligned}
$$

to obtain the equations:

$$
\begin{aligned}
i \dot{c}_{1} & =-\frac{1}{2}\left(\chi_{12} e^{-2 i \omega t}+\chi_{21}^{*}\right) c_{2} \\
i \dot{c}_{2} & =\left(\omega_{21}-\omega\right) c_{2}-\frac{1}{2}\left(\chi_{21}+\chi_{12}^{*} e^{2 i \omega t}\right) c_{1}
\end{aligned}
$$

The terms $e^{ \pm 2 i \omega t}$ oscillate rapidly compared with the other time variation in the equations and can be assumed to average to zero over any experimentally 
accessible time interval and can therefore be neglected. This is known as the rotating-wave approximation (RWA). Using the definition of the detuning $\Delta$ and $\chi$ as given above the differential equations become considerably simplified as:

$$
\begin{aligned}
i \dot{c}_{1} & =-\frac{1}{2} \chi^{*} c_{2} \\
i \dot{c}_{2} & =\Delta c_{2}-\frac{1}{2} \chi c_{1}
\end{aligned}
$$

Using these $c$ 's and defining the density matrix elements $\rho$ as products of $c$ 's

$$
\rho_{m n} \equiv c_{m} c_{n}^{*}
$$

it can be shown that a measurement of an observable $A$ of such a system will result in an ensemble average expectation value of

$$
\overline{\langle A\rangle}=\sum_{n}(\rho A)_{n n}
$$

Of main interest is here the observable induced polarization $P(t)$, as the source term for generated radiation.

With these properties of the density matrix, describing the properties of an ensemble of $N$ identical molecules, each with $n$ states, is reduced from a $0.5 N^{n}$ sized set of equations, to a $n^{2}$ sized set of equations. Since, for a specific set of physical circumstances, many of the states can be neglected, such as was done above for the case of the two-level system, the number of equations can often be reduced even further. The density matrix model is especially suited for those cases where the optical properties of the material are influenced by the light fields themselves.

\subsubsection{Two-level system}

To recall the physical meaning of the described density matrix, let us look at the simple set of density matrix equations derived from eq. 2.18 via 2.20 that describe the standard response of two level atoms driven by a resonant light field, i.e., with $\Delta=0$ (see fig. 2.2 (left)):

$$
\begin{aligned}
\dot{\rho}_{11}(z, t) & =-\frac{i}{2}\left(\chi \rho_{12}-\chi_{12}^{*} \rho_{12}^{*}\right)+\rho_{22} R_{21} \\
\dot{\rho}_{22}(z, t) & =\frac{i}{2}\left(\chi \rho_{12}-\chi_{12}^{*} \rho_{12}^{*}\right)-\rho_{22} R_{21} \\
\dot{\rho}_{12}(z, t) & =\frac{i}{2}\left(\chi^{*}\left(\rho_{22}-\rho_{11}\right)\right)-\rho_{12} \beta_{12}
\end{aligned}
$$

The diagonal elements of the density matrix, $\rho_{i i}$ (eq. 2.22 and 2.23), are the population probabilities of the energetic states of the medium. The off-diagonal 
$|2\rangle$

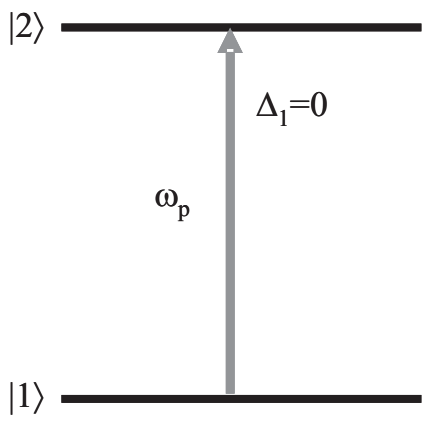

$|3\rangle$

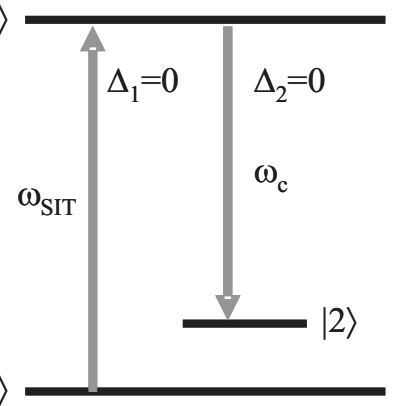

Figure 2.2: Schematic representation of a two-level system (left) driven by a single light field with frequency $\omega_{p}$ and a $\Lambda$-type three-level system (right) driven by two light frequencies $\omega_{S I T}$ and $\omega_{c}$. The latter is driven by two light fields which, in the example, are chosen as doubly single-photon resonant $\left(\Delta_{1}=\Delta_{2}=0\right)$ and thus also two-photon resonant with the $|1\rangle-|2\rangle$ transition.

element $\left(\rho_{12}\right)$, also called atomic coherence, describes with which amplitude and phase the center of gravity of the electron cloud oscillates as driven by the incident light and the atomic restoring force. Note that the coherence can build up and change under the influence of the driving field (expressed through $\chi$ ) in eq. 2.24 in time only when there is a non-zero population difference on the corresponding transition $\left(\rho_{22}-\rho_{11}\right.$ in eq. 2.24). It is the simultaneous presence of a population difference and the radiation field which generates the coherence, which generates the polarization of the medium [44]. In the equations 2.22-2.24 one can also see that decay rates are added for the population decaying from $|2\rangle$ to $|1\rangle\left(R_{21}\right)$ and for the coherence $\left(\beta_{12}\right)$. The decay rate $R_{21}$ accounts for depletion of the upper state through decay to the lower state, such as by the spontaneous emission. Decay of the coherence between states $|1\rangle$ and $|2\rangle$ is given by the rate, $\beta_{12}$, representing elastic damping processes, such as collisional dephasing. Note that the properties of the ensemble of two-level atoms and the drive field is space and time dependent, which means that an incoming light wave drives a population wave and a polarization wave through the medium. Thus calculating the evolution of eq. 2.22-2.24 such as by numerical evaluation would involve, e.g., a grid of points in space and time at which the atomic state is calculated.

\subsubsection{Rabi oscillations}

As an illustration of the temporal evolution of the density matrix equations and to provide an easier interpretation of the results in section 5.4 we recall how a weakly damped two-level system responds to monochromatic light via so-called Rabi oscillations. For such oscillation to occur over many cycles, the decay rates 


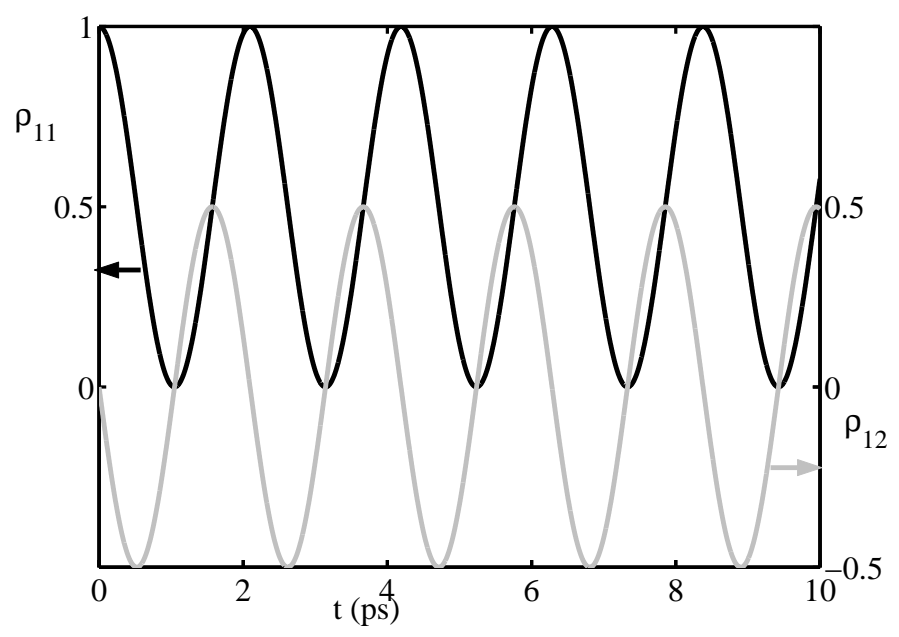

Figure 2.3: Evolution of the population of the lower state $\left(\rho_{11}\right)$ and coherence $\left(\rho_{12}\right)$ of the two-level system when irradiated by a constant field of strength $\chi=3 \cdot 10^{12} \mathrm{rad} / \mathrm{s}$ on resonance with the transition frequency. After $t=0$, when all population is initially in the ground state and the coherence is zero, the magnitude of the coherence starts to grow proportionally with time. This growth is possible because, initially, there is a population difference $\left(\rho_{11}=\right.$ $1, \rho_{22}=0$ thus $\rho_{11}-\rho_{22}=1$ ). Due to the growth of the coherence also the population becomes excited from state $|1\rangle$ to state $|2\rangle$. The magnitude of the coherence is maximum when the population is evenly distributed between the two levels $\left(\rho_{11}=\rho_{22}=0.5\right)$, such as seen at $t \approx 0.5$ ps. Thereafter, the magnitude of the coherence begins to decrease because $\rho_{11}-\rho_{22}$ becomes negative. At maximum inversion $(t \approx 1 \mathrm{ps})$, when $\rho_{11}=0$ and $\rho_{22}=1$, the coherence is again zero, and the population inversion multiplied with the light field, builds up coherence with a reversed sign (antiphased to the previous) and the population moves back towards state $|1\rangle$. 
need to be sufficiently small compared to the driving field, i.e., $R_{21}, \beta_{12} \ll \chi$, which can be fulfilled, e.g., with a sufficiently high drive intensity. To fulfill this in our example we have assumed zero decay rates. Further, to restrict to the simplest situation the detuning of the field frequency from resonance with the transition frequency is taken as $\Delta=0$. As can be seen in fig. 2.3, if initially, at $t=0$, only the ground state is populated $\rho_{11}=1$ the ground state becomes periodically depleted. In addition (not shown) there is an anti-phased oscillation of the upper state population because $\rho_{11}+\rho_{22}=1$. This oscillatory behavior thus consists of a periodical exchange of population from one state to another and back, caused by the incident light field and it can be shown that this oscillation occurs with an angular frequency $\chi=3 \cdot 10^{12} \mathrm{rad} / \mathrm{s}$. Also, there is an oscillation of the envelope of the atomic coherence, $\rho_{12}$, going through its extrema when the populations of $|1\rangle$ and $|2\rangle$ are equal. When analyzing the dynamics in the more general case of a non-zero detuning one finds that the frequency of these Rabi oscillations is not $\chi$ as given by the amplitude of the light field $(E)$, see eq. 2.12 but that a non-zero detuning increases the oscillation frequency to

$$
\Omega_{R}=\sqrt{\chi^{2}+\Delta^{2}}
$$

where $\Omega_{R}$ is called the generalized Rabi frequency. Although $\Omega_{R}$ can easily be made very large by choosing a large detuning, this also reduces the amplitude of the Rabi oscillation by a factor $\frac{\chi^{2}}{\chi^{2}+\Delta^{2}}[44]$.

Fig. 2.3 illustrates the atomic response on a continuous-wave drive field where the Rabi frequency is a constant. For discussing the atomic response also on pulses of resonant light, McCall and Hahn introduced the concept of the so-called pulse area defined as the integral of the drive field over time in terms of the number of Rabi cycles that the pulse drives [35]. For example, if one full Rabi-cycle is induced by a pulse, such a pulse is called a $2 \pi$-pulse.

\subsubsection{Maxwell equations}

The Taylor expansion of the induced polarization in eq. 2.1 and also the induced polarization obtained from the density matrix equations form only one part of the mutual interaction of light with matter, that is, they describe how a medium responds to the presence of a light field such as was shown above at the example of Rabi oscillations. For a full description of light matter interaction it is required to include, e.g., that the medium can be active in the sense that it can generate also a response of the light field, i.e., it generates, attenuates or otherwise modifies the light field. To describe this second effect in its most simple, one-dimensional and scalar form, the Maxwell equations can be used to derive the so-called inhomogeneous wave equation, which describes how the light field propagates and how it is driven by the polarization density $P$ of the medium:

$$
\left(\frac{\delta^{2}}{\delta z^{2}}-\frac{1}{c^{2}} \frac{\delta^{2}}{\delta t^{2}}\right) E(z, t)=\frac{1}{\epsilon_{0} c^{2}} \frac{\delta^{2}}{\delta t^{2}} P(z, t)
$$


Here, $\epsilon_{0}$ is the free permittivity of the vacuum and $c$ the speed of light in vacuum. The polarization of the medium is related to the coherence term of the density matrix equations by:

$$
\frac{1}{\epsilon_{0} c^{2}} \frac{\delta^{2}}{\delta t^{2}} P(z, t)=\frac{i k}{\epsilon_{0}} N \mu_{12} \rho_{12}^{*}
$$

where $k$ is the wavenumber of the light field, and $N$ the number density of the active medium and $\mu_{12}$ is the product of $r_{12}$ and the electron charge and is called the transition dipole moment. The wave equation can be further simplified if the amplitude of the field $E(z, t)$ varies only weakly per optical cycle and propagation wavelength:

$$
\begin{aligned}
\left|\frac{\delta E}{\delta z}\right| & \ll k|E| \\
\left|\frac{\delta^{2} E}{\delta z^{2}}\right| & \ll k\left|\frac{\delta E}{\delta z}\right| \\
\left|\frac{\delta E}{\delta t}\right| & \ll \omega|E|
\end{aligned}
$$

Applying these approximations to eq. 2.26 and eq. 2.27, which is called the slowly varying envelope approximation (SVEA), the wave equation is reduced to [44]:

$$
\left(\frac{\delta}{\delta z}+\frac{\delta}{\delta c t}\right) E=i \frac{N k \mu}{\epsilon_{0}} \sum \rho_{i j}^{*}(z, t)
$$

Here we have replaced $\rho_{12}$ by $\sum \rho_{i j}^{*}(t)$, the sum of the radiative coherence terms to extend the description beyond two-level systems, where radiation may be generated by various additional dipole allowed transitions. The time derivative can be discarded [44] to arrive at the final form of the Maxwell equations as we will use them for the remaining parts of the thesis:

$$
\frac{\delta E}{\delta z}=i \frac{N k \mu}{\epsilon_{0}} \sum \rho_{i j}^{*}(z, t)
$$

From this equation it can be seen that the coherence terms from the density matrix equations on the right hand side act as the source term for the change in the electric field as radiated (absorbed or phase-shifted) by the medium on the left hand side.

\subsection{4 $\Lambda$-type system}

In the following chapters the density matrix model of a three level system in the $\Lambda$-type configuration (see fig. 2.2) is used to model the CARS emission process (chapter 4 and 5) and the propagation of optical solitons (chapter 6 
and 7). For the first case, the applied light fields are far detuned from the dipole transition frequencies, whereas, for the latter case, the light fields are on resonance. However, both of these cases share the same set of density matrix equations, which are as follows [47]:

$$
\begin{aligned}
\dot{\rho}_{11}(z, t)= & -\frac{i}{2}\left(\chi_{13} \rho_{13}-\chi_{13}^{*} \rho_{13}^{*}\right)+\rho_{33} R_{31} \\
\dot{\rho}_{22}(z, t)= & -\frac{i}{2}\left(\chi_{23} \rho_{23}-\chi_{23}^{*} \rho_{23}^{*}\right)+\rho_{33} R_{32} \\
\dot{\rho}_{33}(z, t)= & \frac{i}{2}\left(\chi_{13} \rho_{13}+\chi_{23} \rho_{23}-\chi_{13}^{*} \rho_{13}^{*}-\chi_{23}^{*} \rho_{23}^{*}\right)+ \\
& -\left(R_{31}+R_{32}\right) \rho_{33} \\
\dot{\rho}_{12}(z, t)= & \frac{i}{2}\left(\chi_{13}^{*} \rho_{23}^{*}-\chi_{23} \rho_{13}\right)-\beta_{12} \rho 12 \\
\dot{\rho}_{13}(z, t)= & \frac{i}{2}\left(\chi_{13}^{*}\left(\rho_{33}-\rho_{11}\right)-\chi_{23}^{*} \rho_{12}\right)-\beta_{13} \rho_{13} \\
\dot{\rho}_{23}(z, t)= & \frac{i}{2}\left(\chi_{23}^{*}\left(\rho_{33}-\rho_{22}\right)-\chi_{13}^{*} \rho_{12}^{*}\right)-\beta_{23} \rho_{23}
\end{aligned}
$$

In chapter 5 this set of density matrix equations is further extended to model a four-level system.

\subsubsection{Light field propagation}

In order to study the propagation of pulses and their interactions in $\Lambda$-type media over much longer propagation distances than a single infinitesimal distance $d z$ as in eq. 2.30 considered before, and to allow also for the investigation of media with a high optical thickness or opacity, the density matrix equations (eq. 2.31-2.36) were integrated simultaneously with the integrating Maxwell equation (eq. 2.30) as a set of mutually coupled nonlinear, partial differential equations. The propagation of these pulses was calculated via such integration in the programming environment of $\mathrm{C}++$ using home-built solvers that use both a fourth order Runge-Kutta algorithm, and the Euler method [48].

In chapter 7 we will use this model to theoretically investigate a novel possibility of the storage and mutual interaction of light pulses for the purpose of optical computing. The devised scheme is chosen to be based on a special type of pulses, so-called optical solitons, because these can propagate without distortion and loss through the medium. Also, to enable a strong mutual interaction within a short propagation length, we have chosen to investigate resonant soliton pulses traveling in a medium with a high optical thickness, based on so-called Self Induced Transparency (SIT) [35]. Such SIT pulses induce exactly one Rabi-cycle in the medium (a $2 \pi$-pulse), thereby leaving the population fully in the ground state which corresponds to the absence of absorption. To illustrate the influence of the pulse duration in comparison to a limited lifetime of the upper state, $2 \pi$-pulses with a frequency on resonance with 


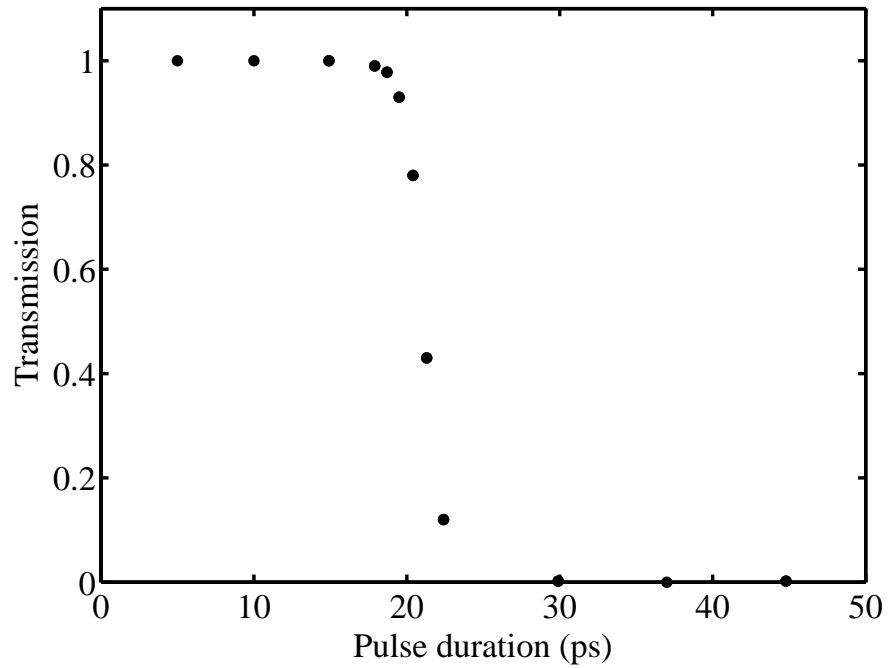

Figure 2.4: Transmission of $2 \pi$-pulses through a $\Lambda$-type medium in which the lifetime of the upper state is set to 200 ps. Pulses with a long duration are strongly absorbed by the medium whereas pulses with a sufficiently short duration propagate without significant loss. 
one of the transitions of a $\Lambda$-type medium and pulse durations ranging from 5 to $45 \mathrm{ps}$ were launched into the medium. The lifetime of the excited state was set to $200 \mathrm{ps}$. The total length of the medium was set to $50 \mu \mathrm{m}$ and the absorption coefficient was determined to be $\alpha=2.8 \cdot 10^{5} \mathrm{~m}^{-1}$. The transmission, which we define here as the area of the pulse exciting the medium divided by the area of the pulse that is applied to the medium, was calculated and is shown in fig. 2.4. It can be seen that pulses with too long a duration $(\tau>25 \mathrm{ps})$ are strongly absorbed by the medium. The transmission values obtained are then approaching what is expected from the well-known law of exponential absorption in an optically thick (opaque) medium. However, indeed, pulses with a duration sufficiently short compared to the lifetime of the excited state $(\tau<18 \mathrm{ps})$ propagate without significant loss through the medium.

By using the coupled set of density and wave equations, the propagation characteristics of solitons and their interaction with an additional control field is studied in chapter 6 . In chapter 7 this model is used to study more elaborate pulse sequencing schemes where the interactions between a set of solitons and their propagation are studied. 


\section{3 \\ Polarization dependent SHG for powder sizing}

\subsection{Motivation}

Although there are many methods to measure the size distribution of powders, such as microscopy [5,6], laser diffraction [8] or dynamic light scattering $[6,7]$, these existing methods suffer from numerous disadvantages when applied in industry during processing (such as milling, granulation, mixing and compaction). The techniques of laser diffraction and dynamic light scattering both require sampling and that the particles be diluted and suspended in a nonsolvating fluid. For industrial processes where a variety of chemicals are used, a non-solvating fluid needs to be found for each chemical. Subsequent measurement of different chemicals requires the measurement device to be cleaned and filled with different fluids each time, which is time consuming. Additionally, mixtures of compounds become problematic because a common non-solvating fluid must be used. Another disadvantage is that the materials used in the measurement process cannot be returned to the production process. Furthermore, these measurements are not performed in real-time, causing additional delays in the production process. Microscopy requires image analysis software to acquire particle sizes from irregularly shaped particles and, if a neural network is used, a large data training set is needed [9]. Each new production process step needs thorough testing and validation before the microscopy technique can be applied, which is compounded by production processes that consist of a large variety of processing steps or chemicals.

In this chapter, a new particle sizing method is demonstrated, based on measuring the intensity of optical second harmonic generation (SHG) from 
individual grains in a powder into which ultrashort pulses are sent to generate the SHG. This measurement technique can be considered to be a $1 \mathrm{D}$ version of an SHG microscope. Unlike earlier measurements [49], it is shown that the grain size can be obtained independently of the absolute SHG power by analyzing the polarization dependence of SHG while scanning the incident laser beam across the powder surface. This makes the method insensitive to intensity fluctuations, which may be caused by a number of factors such as variation in the intensity of the pump beam at the sample, surface roughness of the grains and the so-called phase matching conditions within the grain. Importantly, our SHG particle size measurement does not require additional processing steps such as dilution of the powder, and thereby meets an important requirement for inline, real-time particle sizing.

\subsection{Introduction}

An SHG microscope is based on imaging the scattered SHG signal from a sample and was first reported by Hellwarth et al. [50, 51]. As described in chapter 2, the process of SHG occurs in crystals which belong to the class of non-centrosymmetric structures. Furthermore, SHG also occurs at surfaces and interfaces $[52,53]$.

SHG microscopy has been used to study optical nonlinear responses from a wide variety of samples such as bulk crystals $[54,55]$ to image crystal structures and orientations which are difficult to observe using regular microscopy techniques. Furthermore, the polarization of ferroelectric thin films has been characterized using SHG microscopy [56]. Also biological samples have been studied where SHG has been used to image at a depth within the tissue. In this case, SHG imaging revealed the orientation of muscle cells $[57,58]$. By varying the focal depth of the laser spot in the sample, the SHG imaging technique has been expanded to three spatial dimensions by Gauderon et al. [59]. Without mapping the spatial information, using only the integrated SHG intensity, the formation of the crystalline structure has been measured during deposition of a thin film [60], and to study electronic polar reconstructions at interfaces of thin films [61]. As an expansion to the imaging capabilities a rotation of the polarization angle of the incident light has been used to study, for instance, the orientation and presence of optical nonlinear axes of a single crystallite in order to determine its crystal class [62] and the orientation of collagen in a biological sample [63].

However, no reports have been found in literature that use polarization dependent SHG for sizing particles in a powder. Polarization dependent SHG measurements provide a significant advantage over simple intensity measurements, as illustrated below. A laser beam, focused to a spot size that is smaller than the particle size, will be generate an SHG signal that depends on the orientation of the crystal relative to the polarization of the laser beam, the path length of the light through the crystal, and the scattering modes, as determined by the shape of the particle. Scanning the laser beam along the particle changes 
all of these parameters, except for the orientation of the crystal relative to the polarization of the laser beam. By analyzing the polarization dependence of the SHG efficiency as a function of laser beam location, changes in crystal orientation can be detected with high sensitivity. Since, in a random medium, there is a low probability of encountering two crystals (we assume that the crystals are single domain, which is generally safe for crystals of organic molecules) with the same orientation beside each other, it is possible to determine crystal edge locations very accurately. The distance between these locations can then be used to generate distributions related to the size of the particles.

There are various different experimental realizations of SHG microscopes. For instance, the transmitted SHG light can be detected $[50,54]$, the backscattered light [57] or the backscattered light under an angle (see fig. 3.1). Furthermore, an image can be formed either by illumination of the whole sample and detection with a CCD camera [55] or by scanning and detection with a photomultiplier tube (PMT) and reconstructing the image by combining the measurement with the scanning position [57].

The experimental setup, which is described in detail in the following section 3.3, involves an infrared laser producing ultrashort pulses as the light source, which we name the pump source. The polarization angle of the pump light is set by a waveplate and the pulses are then focused on a cuvette on a translation stage containing the powder under investigation. A motor moves this translation stage and the backscattered SHG light as well as the backscattered pump light are measured simultaneously as a function of scanning position and polarization angle. Measurements were performed on single particles and on powders. These measurements show that the backscattered pump light as a function of scanning position from a single particle can be readily interpreted and reveals the chord length over which the particle was scanned. However, the backscattered pump light from a powder cannot be unambiguously related to the chord lengths of the individual particles in the powder due to the increase in background signal. In contrast, it will be shown that the analysis of the polarization dependent SHG intensity, from both single particle samples and powder samples, provides the chord lengths of individual particles.

Using a numerical model it will be shown that the mean and median values of the set of chord lengths is related to the size and shape of the particles, using the assumption that the shape of the particles is to the first order approximation an ellipsoid and has a monodisperse distribution. Using this approach the measured chord lengths as obtained by the scanning method are related to the physical size aspects of the powders. Furthermore, the number of chord lengths needed to obtain an accurate average particle size from a monodisperse powder is calculated. Using the experimental setup as described in this chapter and the number of measurements that have been performed, only values for the mean particle size of powders were obtained. A comparison between particle size distributions obtained from a commercially available instrument and SHG scanning are beyond the scope of the research presented in this chapter. Such comparisons are, in any case, difficult, due to the different physical techniques 
used by different instruments. However, extensions or improvements to the experimental setup may be implemented in future to enable such a comparison.

In section 3.3 we describe the experimental setup and introduce the data analysis methods which are tested for a single grain (section 3.4.1) and sets of single grains (section 3.4.2). In section 3.3.2 we show that different polarization dependent traces are recorded for powders belonging to different crystal classes. In section 3.4.3 we show that the measurement technique is surface sensitive and that it provides considerable advantages over ordinary microscopy. In section 3.4.4 we provide examples of particle sizing measurements on $\alpha$-lactose powders.

\subsection{Experimental}

The measurement technique, which maps the SHG intensity as function of the polarization angle of the pump light and the position of the focus of the beam, will be described in section 3.3.1. The retrieved SHG signal for each position along the path and for each polarization angle of the light is then analyzed using an algorithm (section 3.3.2) that provides information on the length of the grains and crystal class.

\subsubsection{Optical setup}

As shown in fig. 3.1, the basis of the experimental setup is a Kerr lens modelocked Ti:Sapphire laser (KM Labs Model TS Ti:Sapphire laser kit, $800 \mathrm{~nm}$, $50 \mathrm{fs}, 300 \mathrm{~mW}, 80 \mathrm{MHz}$ ). The linearly polarized laser beam is passed through a half-wave plate to set the polarization angle. To ensure that the laser beam is mostly incident on a single grain in the sample, it is focused to a $20 \mu \mathrm{m}$ (FWHM) spotsize by a lens $(f=80 \mathrm{~mm})$. The sample is held in a plastic cuvette and is linearly translated up to $6 \mathrm{~mm}$ at a speed of $50 \mu \mathrm{m} / \mathrm{s}$ orthogonally to the angle of incidence of the laser beam. The scattered light is collected by an aspheric lens $(f=40 \mathrm{~mm}, \mathrm{NA}=0.55,50 \mathrm{~mm}$ diameter $)$ placed $5 \mathrm{~cm}$ from the point where the laser beam is incident on the sample. The collimated output from the aspheric lens is focused by another lens $(f=100 \mathrm{~mm})$ so that all the collected light is incident on the detectors. The infrared and its second harmonic are separated by a dichroic mirror (Thorlabs FM04). The infrared is detected by a photodiode (BPW 34B), while the second harmonic passes through a bandpass filter $(T=30 \% 390-400 \mathrm{~nm})$ and is detected by a photomultiplier tube (PMT) (Hamamatsu H6613). The signals from the photodiode and PMT are recorded simultaneously by an oscilloscope with identical lowpass RC filters $(R=1 \mathrm{k} \Omega, C=1 \mu \mathrm{F})$. A complete measurement run consists of measuring the fundamental and SHG power as a function of position for either nine or eighteen different polarization angles between 0 and 180 degrees. Each measurement starts and ends at approximately the same position as will be described further in section 3.3.2. 


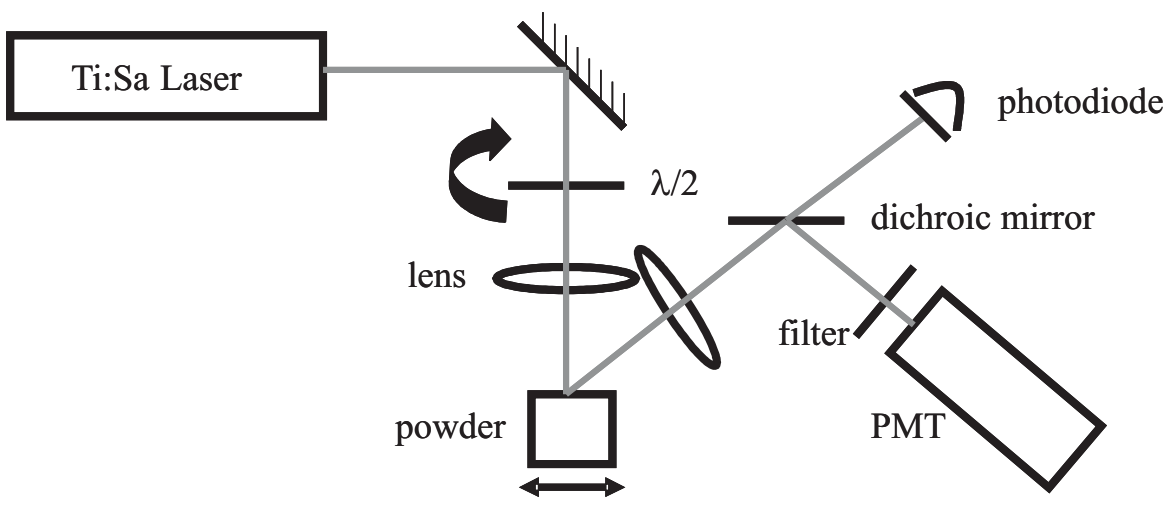

Figure 3.1: Schematic overview of the optical setup of the polarization dependent SHG scanning measurements. The polarization angles of the ultra short pulses from the Ti:Sa laser are varied by rotating the $\lambda / 2$ waveplate. The beam is focused tightly onto the sample by a lens. The scattered light from the powder sample, containing the pump and SHG light, is collected by a second lens. The dichroic mirror transmits the pump light which is detected by the photodiode and reflects the SHG light which is detected by a photomultiplier tube (PMT) simultaneously. A chromatic filter is placed in front of the PMT in order to absorb excess pump light.

Measurements of the polarization dependent SHG signal of a single grain and a set of single grains, affixed to a piece of transparent tape, were carried out as an initial test. This test shows that the extent of regions with a similar polarization dependent SHG signal is the same as the physical length of the grain over which it has been scanned (section 3.4.1 and section 3.4.2). These include measurements on single particles, on a few single particles and on powders, of both $\alpha$-lactose and quartz. Furthermore, it will be shown that measurements from bulk powders are dominated by the particles on the front surface by comparing measurements of a single layer of particles with the same layer in a bulk powder (section 3.4.3).

\subsubsection{Data analysis}

The amount of data that is recorded and subsequently analyzed depends on the spatial resolution, the length of the scan and the number of different angles of polarization. The calculated spatial resolution of the scan for these measurements is $50 \mathrm{~nm}$ as set by the RC time of $0.1 \mathrm{~ms}$ of the low-pass filter and the translation speed of the sample of $50 \mu \mathrm{m} / \mathrm{s}$. Typically the sample is scanned over a few millimeters, limited by the range of the translation stage and the 
dimensions of the cuvette. The number of different angles of polarization was set to 9 for the measurements on $\alpha$-lactose and 18 for the measurements on quartz. The measurement data is processed using an in-house algorithm.

For the experimental realization of the measurements on powders varying both spatial position and polarization angle, we chose to fix the polarization angle while scanning the powder across the focus of the beam. This process was repeated for a set of polarization angles by scanning the same path each time. The process, however, requires that the polarization dependent data from each spatial location must be combined at the end of the total measurement. For this purpose, each translational scan consists of scanning the sample in both the forward and reverse directions. The accuracy of the motor ensures that the start and end-point of each scan is identical to within $100 \mathrm{~nm}$. We use the end-point of the scan, around which the scan data should be a mirror image, to align the scan data from each polarization.

In practice, the mirror point is found by searching for correlations in the data. Specifically, the data is divided into two sets at some location and the cross correlation between the two sets is measured. The location at which the data is divided is then varied until the cross correlation is maximized. This location then becomes the end-point of the scan data. This process is repeated for the set of data of each polarization angle, allowing the complete set of data to be aligned by overlapping these mirror points. An example of aligned data of polarization dependent SHG measurements for $\alpha$-lactose and quartz powder samples is shown in fig. 3.2.

The polarization dependence of the SHG from the aligned set of data must now be analyzed to determine the chord lengths. Two different analysis techniques were developed: one based on directly fitting the polarization dependence to a physical model, while the second was on finding regions with a high cross-correlation coefficient. Each algorithm has strengths and weaknesses depending on the purpose of the analysis and will be discussed in the remainder of this section.

\section{Algorithm based on sinusoidal fit-functions}

As described in section 2.1.4 the shape of the polarization dependent signal is determined by the crystal type and its orientation. As an example of the difference in polarization dependent signal from two different crystal classes, a typical example of such signals for $\alpha$-lactose and quartz is shown in fig. 3.3.

By fitting the appropriate function (depending on the crystal class) to the set of polarization dependent data of each lateral position, the best values of the fit for $\alpha$ and $\theta$ are obtained. It is expected that adjacent single crystalline grains, having a random crystal axis orientation, will have different values of the best-fit-coefficients. Therefore, by observing the similarity between these bestfit-coefficients at adjacent points along the scan direction, an estimate of the chord length over which a single grain is scanned can be made. The threshold value for the "decision" whether the measurements from two adjacent regions 

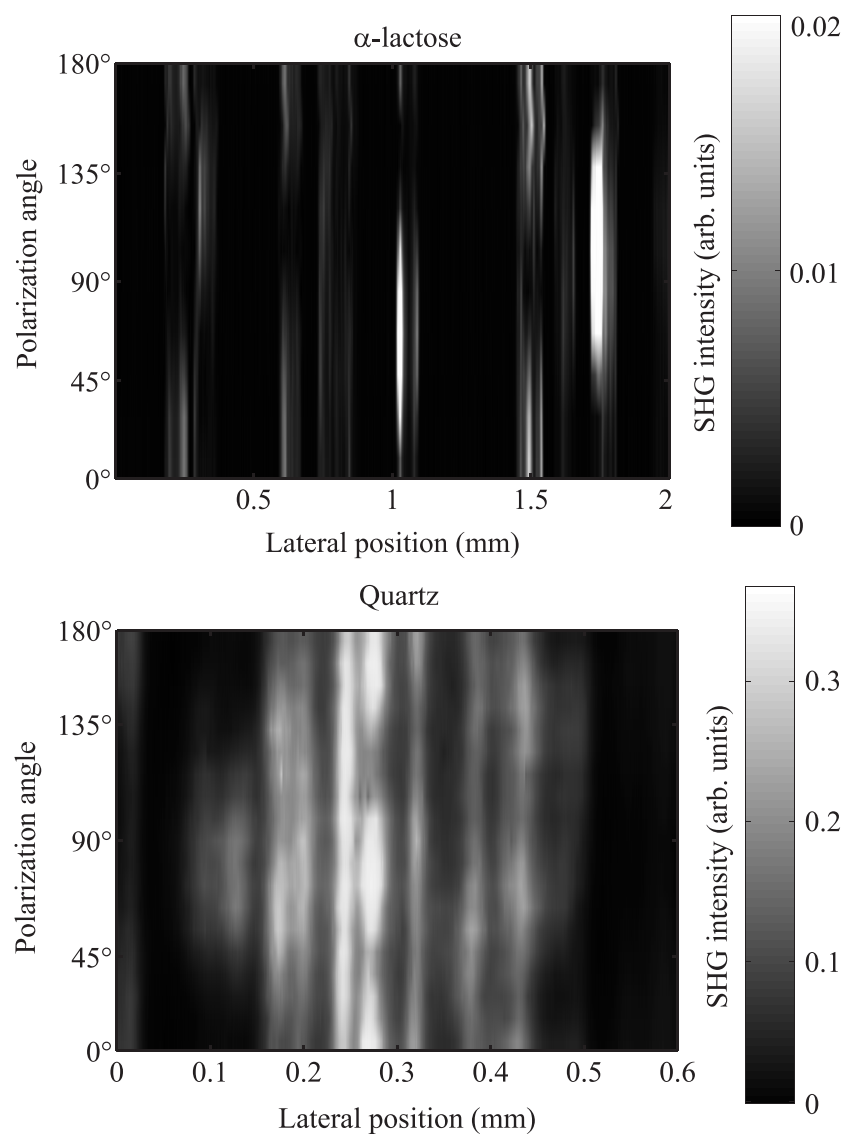

Figure 3.2: (upper) Measurement of the power of the SHG signal as a function of polarization angle of the laser light and lateral position for a powder sample of $\alpha$-lactose. Connected regions with a similar polarization dependency of the measured power indicate the presence of a single crystalline grain. Each grain has a single optimal polarization angle which is a consequence of the second order nonlinear properties of the crystal class of $\alpha$-lactose. (lower) Measurement of the power of the SHG signal as a function of polarization angle of the laser light and lateral position for a powder sample of quartz (notice that a different scale on the $x$-axis was used to make a better visualization of the fine structure of the patterns). Connected regions with a similar polarization dependency of the measured power indicate the presence of a single crystalline grain. Some grains, but not all, have two semi-optimal polarization angles which is a consequence of the second order nonlinear properties of the crystal class of quartz. 


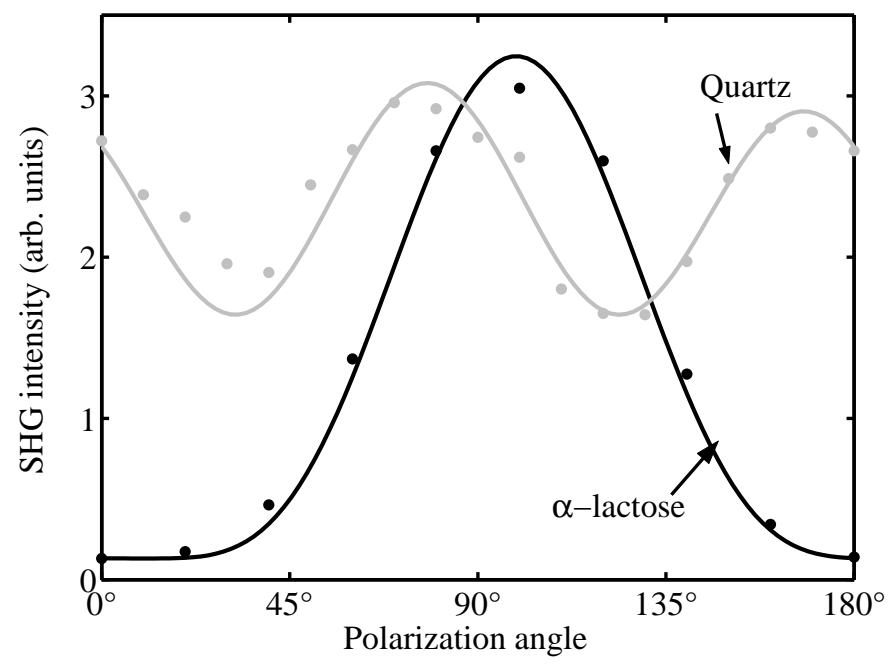

Figure 3.3: Measured SHG intensity as a function of the polarization angle of the laser light for two different crystal classes, $\alpha$-lactose (black dots) and quartz (gray dots). The crystal class of $\alpha$-lactose has 1 optical nonlinear axis (the black line is the best fit of a sinusoidal function). The quartz crystal class has 2 nonlinear axes, which, under certain crystal orientations, produces two peaks in the polarization dependent signal (the gray line is the best fit). 
are likely to belong to one grain was determined by comparing the outcome of this algorithm with microscope pictures of a sample as in section 3.4.2.

\section{Algorithm based on correlation coefficients}

Instead of fitting the fit function to each set of data, which is rather time consuming, a faster approach is to use the correlation function. In this algorithm two spatially adjacent sets of polarization dependent data are correlated. The correlation factor provides a quantitative number (between -1 and 1) for the similarity of two traces with respect to their polarization dependency irrespective of the amplitude. A value of 1 is reached for the case of complete similarity and 0 for the case where there is no similarity between the sets of data, while -1 indicates that the data is anti-correlated, which in our case also indicates that the adjacent regions do not exhibit similar polarization responses. This property of the correlation function makes it suitable for analysis of the data, where the polarization dependency reveals the extent of single grains, but where the intensity of the signal may vary strongly for two lateral points on the same grain. By computing the correlation factor between spatially connected regions, the extent of the region, for which the correlation factor exceeds a certain threshold value, can be expected to represent the chord length over which the laser spot has scanned the grain.

The correlation function is defined as:

$$
\rho_{A, B}=\frac{E\left(\left(A-\mu_{A}\right)\left(B-\mu_{B}\right)\right)}{\sigma_{A} \sigma_{B}}
$$

With $A$ and $B$ the two sets of data for which the correlation coefficient is calculated, $E$ is the expected value operator, $\mu_{A}$ and $\mu_{B}$ the mean value of the distributions of $A$ and $B$ respectively and $\sigma_{A}$ and $\sigma_{B}$ the standard deviation of those distributions.

As an example of the correlation coefficient of sets of measurement data, the correlation coefficient between sets of data points obtained by the polarization dependent measurement of the SHG intensity from a quartz sample is shown in fig. 3.4. It can be seen that traces may have a large correlation coefficient for points that are likely to belong to the same grain, and points that are likely not to belong to the same grain do not have large correlation coefficients. Furthermore, the strength of this analysis technique is shown by the example where the amplitude of two traces differs by a factor of three, whereas the shape of the curves is very similar, as is also confirmed by the very high correlation coefficient between those sets of data.

\subsubsection{Relationship between chord length and mean par- ticle size}

The definition of the size of a particle is rather complex, since, in reality, such a grain may assume various odd shapes. In laser diffraction measurements, the 

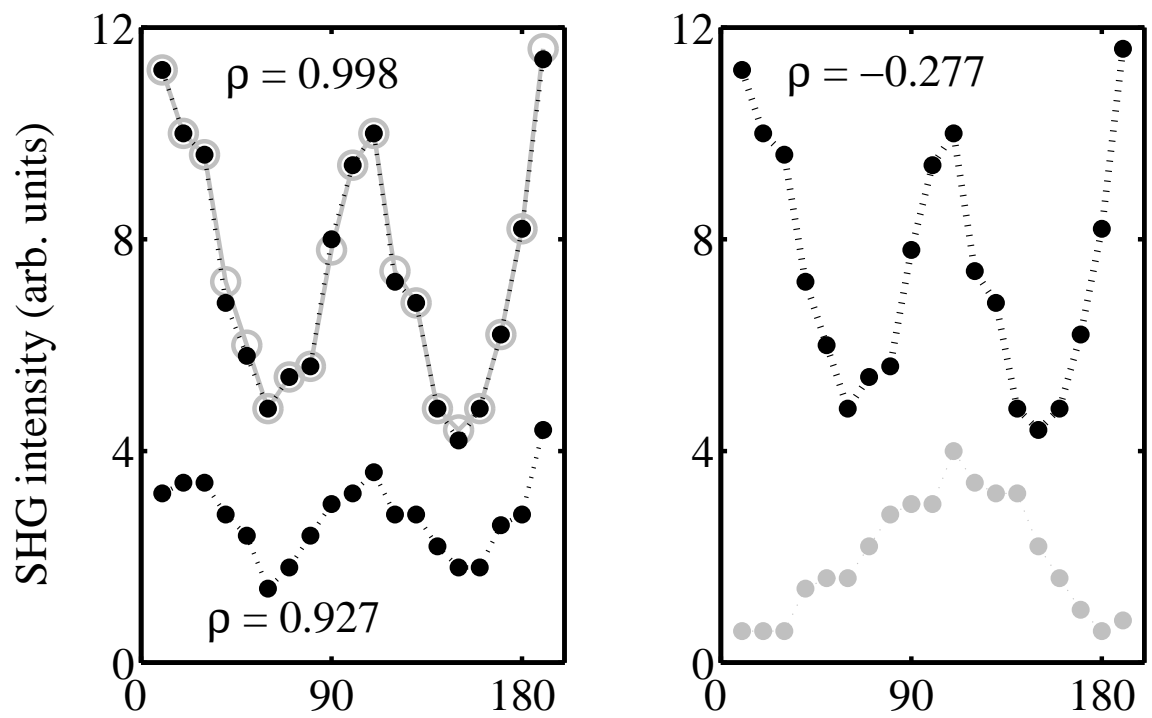

Polarization angle (deg)

Figure 3.4: Examples of the correlation coefficients of sets of measurements. (left) The SHG response as function of the polarization angle between two adjacent positions on the sample $(\approx 100 \mathrm{~nm}$ separation $)$ shown by the upper set of black dots and gray circles. These sets of data have a very high correlation coefficient of 0.998 . The bottom row of black dots was measured at a distance of $5 \mu \mathrm{m}$ with respect to the other data. Although the amplitude is decreased by a factor of 3 , the correlation coefficient between the sets is very high (0.927) which confirms that the shape of the curves (as indicated by the dotted line as a guide to the eye) are very similar. (right) Two sets of data from measurements with a separation of $30 \mu \mathrm{m}$, the curves are not similar in shape, which is confirmed by the correlation coefficient of -0.277 . 
distribution of particles is expressed in terms of volume. This volume can be related to a length and a width of a particle by approximating those particles as spheres or ellipsoids. For the assumption of ellipsoidally shaped particles, as a first approximation, to have a given basic shape, we define here the aspect ratio of a particle as the length of the longest axis divided by the length of the shortest axis.

By scanning the powder sample with the polarization dependent SHG setup, the chord length over which separate grains where scanned can be obtained. The length of such a chord can assume any value between 0 (merely skimming the particle) and the longest axis of the grain. Therefore, a measured chord length, by itself, will only determine a lower bound on the size of the particle. As will be shown by combining a large set of measurements of such chord lengths, the average or median value and spread of the distribution of these lengths provides information on the physical size of the grains in the powder.

Through a numerical model, in which a large number of measured chordlengths of an ellipsoidal shaped particle is calculated, the mean and median values for a given aspect ratio are obtained. This numerical model is based on the random selection of the measurement orientation with respect to the fixed coordinate system of an ellipsoidal grain with a set shortest and longest axis. Subsequently a chord across the grain is randomly selected. This process is then repeated a 10,000 times to generate the chord-length statistics.

The results of this numerical model are presented in fig. 3.5 for various aspect ratios of ellipsoidal shaped particles, where it can be seen that each mean or median value of the distribution of measured chord lengths relates to one specific aspect ratio. Therefore, if the aspect ratio of the particles in a monodisperse powder is known, the mean or median value of the chord length gives a good estimate of the grain size. Furthermore, the aspect ratio of a monodisperse powder can be estimated by comparing the mean and median value.

\subsection{Results}

By using the experimental conditions as described in the previous section, several experiments were performed to study the particle sizing method by comparing the obtained grain size distribution with other standard measurement techniques. Furthermore, the polarization dependent SHG measurements were used to show that information about the crystal class of powdered samples can be obtained simultaneously.

The samples consisted of $\alpha$-lactose powder (obtained from the Faculty of Pharmacy, Helsinki University), sieved (Endecotts 150, 106, 72) into five particle size ranges: $<45 \mu \mathrm{m}, 45-71 \mu \mathrm{m}, 71-105 \mu \mathrm{m}, 105-150 \mu \mathrm{m}$, and 150-180 $\mu \mathrm{m}$. And a set of $\alpha$-lactose and quartz powders (obtained from Otago University, New Zealand), each sieved into 4 particle size ranges $<75 \mu \mathrm{m}, 75-150 \mu \mathrm{m}$, 150-250 $\mu \mathrm{m}, 250-355 \mu \mathrm{m}$. 


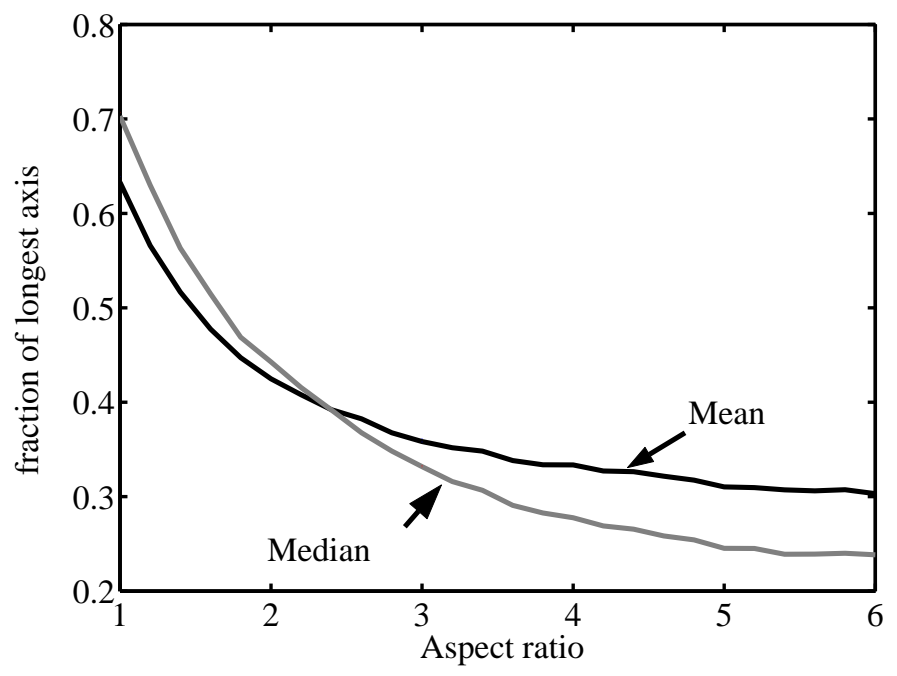

Figure 3.5: The mean and median chord lengths across ellipsoidal grains, as a fraction of the longest possible chord length is given for various aspect ratios (long axis / short axis).

\subsubsection{Single grain experiment}

As an initial experiment to study the polarization dependency of the SHG signal from a well defined sample, a single grain of $\alpha$-lactose was fixed onto a piece of transparent tape. A microscope picture of the sample was taken (see fig. 3.6 (upper)) and the length of its longest horizontal axis was determined to be $400 \mu \mathrm{m}$ by measurement using the size scale of the microscope. This sample was scanned across the longest horizontal axis and the scattered pump and SHG light were recorded (see fig. 3.6 (middle)). In this figure, it can be seen that the width (determined by where the intensity exceeds the background signal) of the peak of the reflected fundamental light is approximately $400 \mu \mathrm{m}$, which is in agreement with the grain size, as revealed by microscopy. In contrast, the SHG signal shows two large peaks rather than one.

The analysis of the polarization dependent SHG (by fitting) showed that the chord length can be revealed. This is illustrated in fig. 3.6 (lower), where the phase of the fitted sinusoid is plotted as a function of position. Note that the phase is constant (the standard deviation over the constant region is approximately $0.1 \mathrm{rad}$ ) over the entire range between both SHG power peaks, indicating that this is indeed a single grain. Furthermore, the absolute distance over which the phase remains constant is $400 \mu \mathrm{m}$, in excellent agreement with microscope results. 


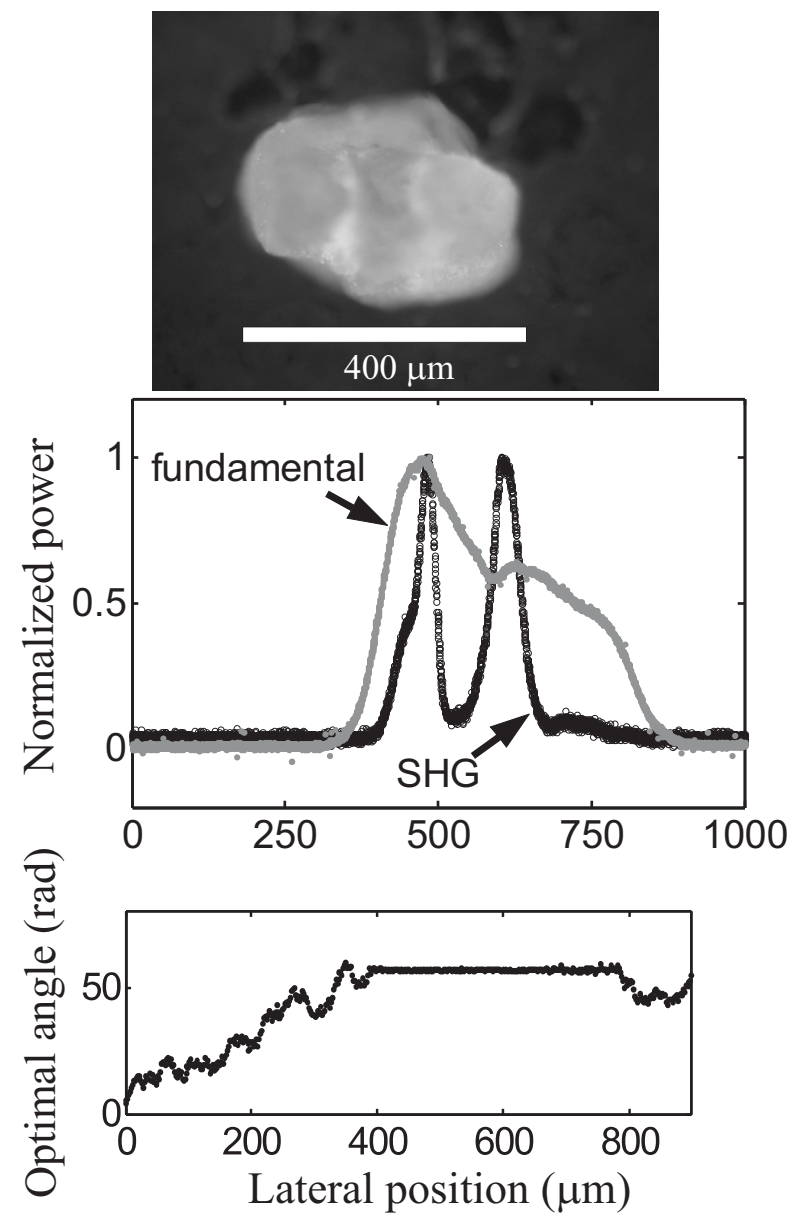

Figure 3.6: (upper) Microscope image of a single grain of $\alpha$-lactose. (middle) Measured intensity of the pump (gray) and SHG (black) power as a function of lateral scanning position over a single grain of $\alpha$-lactose (shown in the upper picture) for a single polarization angle of the laser light. The observed peak in reflected pump light (where the intensity exceeds the average background signal) is a measure of the chord length over the grain and is approximately $400 \mu \mathrm{m}$ which corresponds to its maximum length as obtained from the microscope image. The SHG signal shows two large peaks and a small wing. From this shape one might, erroneously, conclude that the sample consists of two small grains. (lower) By combining the measurement data from repeated scans with various polarization angles, and processing the data to obtain the polarization angle for which a maximum of SHG power is measured, a connected region with a constant optimal angle is revealed. Such a region is an indication that the observed sample consists of a single crystalline grain. The length of this region is an indication of the chord length over this single crystal and corresponds to the maximal chord length of $400 \mu \mathrm{m}$ as obtained from the microscope image. 


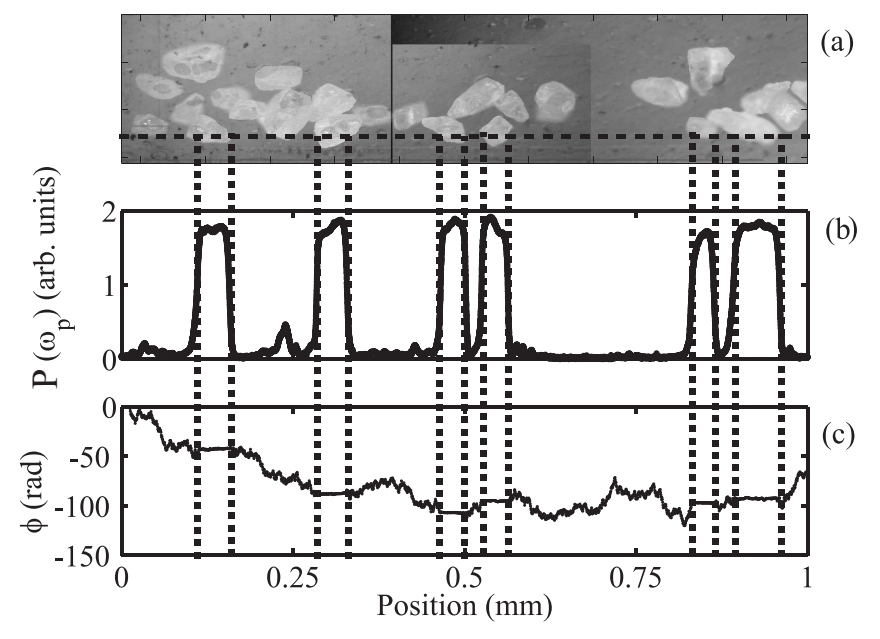

Figure 3.7: (a) Composition of microscope images of a sample of $\alpha$-lactose on a piece of tape. The horizontal dotted line represents the probable scan line of the laser during the polarization dependent SHG measurements. (b) Measured pump light after scattering off the sample as shown in (a). The peaks correspond to the presence of a grain at those locations. (c) The optimal angle of polarization to induce SHG as obtained by combining the data of measured SHG power for a set of various polarization angles. The connected sections with a constant optimal angle correspond to the regions of peaks of reflected pump power and the location of single grains.

\subsubsection{Multiple single grains}

As an intermediate step from a single grain to a powder, a sample where a few grains of $\alpha$-lactose are affixed to a piece of tape was prepared and measured by the polarization dependent SHG setup. A set of pictures were taken by an optical microscope as well (see fig. 3.7 (a)) for comparison with the data as obtained by the SHG microscope measurements.

From these measurements it can be seen that the chord length of several separate particles can be obtained by comparing the scattered pump light (see fig. 3.7 (b)) with the microscope picture. Furthermore, by fitting a sinusoidal function to the polarization dependent SHG data for each position, the optimal angle for SHG is determined (see fig. 3.7 (c)). Similar to the measurements on the single grain, the connected regions with a constant optimal angle (to within $\approx 0.1 \mathrm{rad}$ ) appear to overlap with both the microscope picture and the peaks (where the intensity exceeds the background level) in the reflected pump light. The different optimal polarization angles between grains facilitates the detec- 
tion of more closely spaced particles for cases where changes in the reflected pump light do not reveal these grains, due the large amount of scattering, for instance in powders. This will be shown in the next section.

\subsubsection{Front layer sensitivity in powders}

The light scattered from a powder sample which is scanned by a light source has much less variation in intensity as compared to single grains. This can be explained through the following. For a single grain, light is only scattered when the laser is incident on the particle, therefore a large variation in scattered light intensity is seen when the focused laser beam traverses the particle. However, in a powder, the light is always scattered at some location in the powder due to the large amount of particles. The variations in the intensity of the backscattered light, therefore, become much smaller than for the case of a single grain.

This increase in scattering from a powder sample, as compared to a few grains, makes it very difficult to obtain size ranges from the measured pump light power. In contrast, the efficiency of the SHG process depends quadratically on the intensity [43] and this intensity drops off fairly rapidly within a powder. Therefore, it is expected that the SHG signal originates primarily from the first few layers of grains, where the intensity is high, reducing the influence of scattering from other parts of the powder.

This is shown in the following experiment where a sample was prepared as follows. A metal holder, with openings on the back and front end, was covered by a piece of transparent tape (Pritt Crystal Clear Premium Quality Tape) and filled with quartz powder. This sample was measured by the polarization dependent SHG setup. Subsequently, maintaining the holder in place, the tape on the back surface was removed and excess powder was removed. Only a single layer of particles remained fixed to the piece of tape on the front end. Then, a second set of measurements was performed. Through this procedure the front layer of grains was measured twice, once with grains behind it, to form a powder and once as a single layer, in order to determine the scattering and SHG contribution from grains located below the surface layer. Both the reflected pump light and polarization dependent SHG light were detected.

The measurement of the pump light from the powder (see fig. 3.8 (upper)) shows that no distinct peaks, such as observed from single grains, are observed. Despite the fact that some maxima and minima are coincident in both traces, the significance of the peaks in the black trace is only revealed by comparing both traces. The fluctuations in scattering signal from the powder cannot easily be attributed to features of certain grains, nor can the size of such grains be determined. This is because the dynamic range of the light intensity measured by the photodiode is very small, providing very little margin to, for instance, set a threshold. The gray trace in fig. 3.8 (upper), which shows the scattered pump intensity for just a single layer confirms that the reduction in dynamic range is due to multiple scattering.

The measurement of the polarization dependent SHG signal from the quartz 

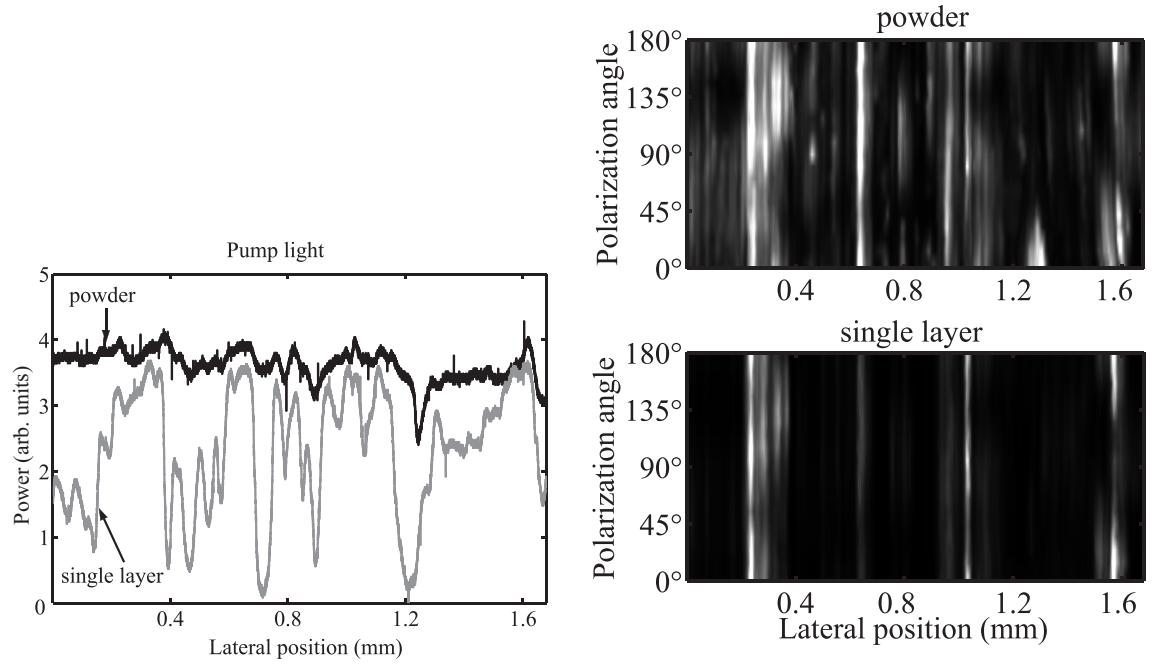

Figure 3.8: (left) Measurement of the power of the reflected pump light as a function of the lateral position during a scan of a quartz sample in the form of a powder (black) and only the first layer of the same powder (gray). By comparing both traces it is observed that the deviation of the signal is much smaller for the case of the powder as compared to the single layer. Some, but not all, features of peaks and dips in the signal from the single layer also occur at the same location for the powder. (right upper) Measured SHG power as a function of polarization angle of the beam and lateral scanning position for an $\alpha$-lactose powder sample. (right lower) Measured SHG power as a function of polarization angle of the beam and lateral scanning position for only the front layer of grains of quartz powder sample. By comparing both samples it is observed that all grains as found in the single layer are also present in the powder, indicating that this measurement technique reveals the properties of the first layer of grains in a powder, without much influence of scattering from subsequent layers. 
powder (see fig. 3.8 (lower)) and the first layer only shows that most features occur for both cases at the same location with a similar dynamic range in the signal. These features consist of regions with similar polarization dependent SHG signals. Such a region is an indication of the presence of a single crystalline grain as was shown in sections 3.4.1 and 3.4.2. Those grains, as revealed by the single layer measurement, are also revealed in the powder measurement. Therefore, the polarization dependent SHG measurement is shown to be sensitive to the first layer of grains only, thereby minimizing a perturbing influence of grains deeper in the powder. Furthermore, regions of the same polarization dependency along the lateral direction provide the chord length of the grain over which it was scanned. This information can be used to characterize the particle size of a powder.

The differences between both graphs can be attributed to a couple of imperfections when performing the experiments. For instance, particles that were in close proximity of the tape, but not stuck to it, may have been measured for the case of the powder but may have fallen away for the case of the measurement on the front layer only. Furthermore, the process of removing the excess powder may have caused some shifts in the position of the grains between both sets of measurements.

\subsubsection{Particle sizing of $\alpha$-lactose powders}

As shown in the previous section, the polarization dependent SHG measurement technique can be used to obtain information of the particle size even in the challenging case of a sample in the form of a powder not diluted in a fluid. For the characterization of the particle size in a powder, a set of measurements was performed on $\alpha$-lactose powders that had been sieved into different size ranges. Each powder has been characterized by two methods, through analysis of optical microscope pictures and through the scanning polarization dependent SHG microscope. The average of the measured chord size (in the case of scanning SHG) and maximum chord length for microscopy) and the standard deviation of the particles in the powder is shown in fig. 3.9. It is found that the particle size ranges agree well with each other, though they differ substantially from the range one might expect from the sieve mesh sizes. Furthermore, as expected, the scanning SHG method reports a similar minimum value in the range for all cases.

\subsubsection{Comparison of sizing methods}

To reduce the uncertainty of the values for the mean particle size and spread of the size distribution, a large set of chord lengths needed to be recorded to find the mean particle size and spread. For this purpose, two different sets of $\alpha$ lactose powder have been characterized by collecting a large set of chord lengths with the polarization dependent SHG technique $(N=200)$. For comparison with standard particle sizing techniques, a laser diffraction measurement was 


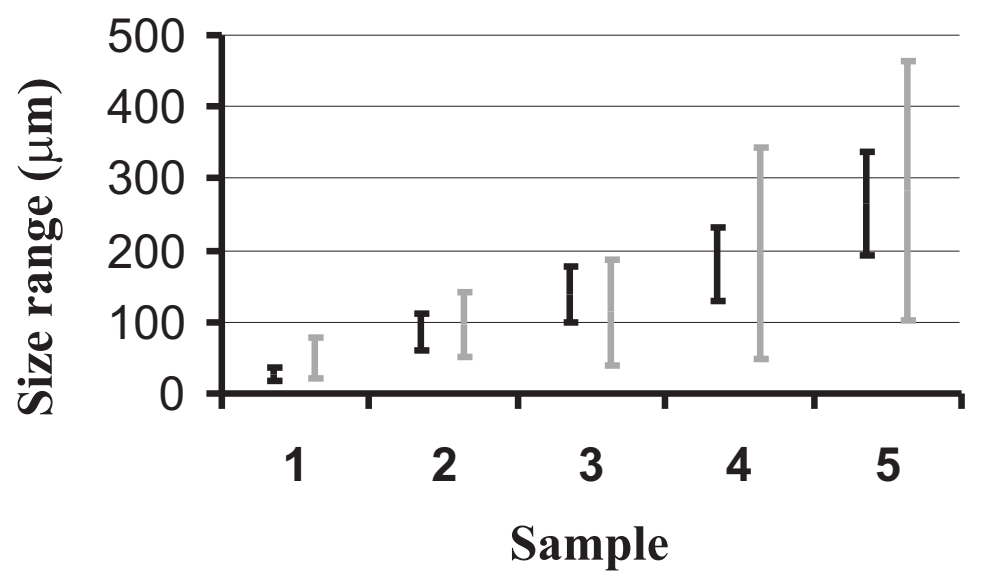

Figure 3.9: Average size of particle sizes in 5 differently sieved powders of $\alpha$ lactose as measured using an optical microscope (black) and after analysis of a polarization dependent SHG measurement (gray). $N=30$.

performed (by Clare Strachan and Pranav Karmwar, University of Otago, New Zealand) on the same set of powders (Horiba LA-950V2). The particle size distribution, obtained by both sizing techniques, are shown in fig. 3.10. In this figure it can seen that the two sets of powders (1) and (2) have different mean particle sizes and standard deviation. By comparing both measurement techniques it can be observed that the mean particle size of sample (1) is smaller than that of sample (2). Although the spread in particle size as obtained by the polarization dependent SHG measurement technique appears to resemble some features like width and position, as obtained by the laser diffraction technique, the curves are not as smooth.

Through modeling of the distribution of random chord lengths across particles, we estimated that a set of 500 chord lengths needs to be obtained to calculate the mean particle size of a monodisperse powder with an accuracy of 1\%. To reduce the amount of time needed to obtain such a large set of data, the experimental procedure requires optimization.

\subsection{Conclusions and outlook}

In this chapter a new approach to measure the size of particles in powders via polarization angle sensitive recordings of the generated second harmonic when scanning a focused laser beam across the powder sample was demonstrated.

By comparing reflected pump light and polarization dependent SHG light 


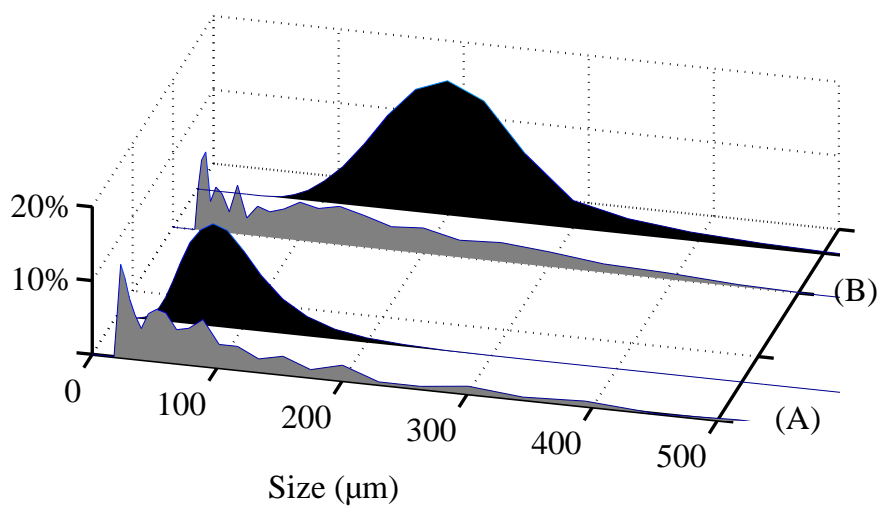

Figure 3.10: Set of histograms of the abundance per grain size in powders of $\alpha$-lactose as measured by laser diffraction (black) and polarization dependent SHG measurements (gray), for two powders that have been sieved with different sieve-mesh sizes.

from a front layer of grains with a full powder, it is concluded from the polarization dependent signal that information can be obtained about the grain size of the sample, whereas no information about the grain size could be obtained from the reflected pump light. This shows that a polarization dependent SHG setup offers the possibility of characterizing the grain size distribution in undiluted powders by using an all-optical measurement setup. The simplicity of this method is interesting for in-line monitoring for the food and pharmaceutical industries.

Furthermore, it was shown that a difference in crystal class between two samples can be identified, by studying the shape of the function describing the SHG intensity as a function of polarization angle. This might be used to obtain mixture fractions of a sample consisting of a mix of powders from different crystal classes.

By further improving the data acquisition speed, a larger set of chord lengths can be obtained, providing a statistically reliable grainsize distribution. Such an improvement can be achieved by mounting the half-waveplate on a motorized rotation stage and by feeding the temporally changing rotation angle and SHG signal to a lock-in amplifier in order to measure the crystal axis orientation. 


\section{4 \\ Numerical modeling of CARS}

\subsection{Introduction}

A crucial part in imaging an object is identification of the constituents of the sample and the formation of a sharp image, specifically, with respect to their chemical components and specific position. All of the currently existing imaging techniques are limited in the obtainable spatial resolution and in resolving the chemical constituents. This limitation in imaging stresses the need to find alternatives or improvements to the current techniques. In the next chapter, we will present two novel schemes that promise to improve the spatial resolution of a spectroscopic imaging technique. This promise is based on results obtained from calculations of a density matrix model. This model, numerical solutions and its validation are presented in this chapter.

The imaging techniques that are most commonly used can be roughly grouped into three classes. The first class are those imaging techniques that use a scanning probe such as to measure the mechanical force in atomic force microscopy (AFM) or to measure a tunnel current in scanning tunneling microscopy (STM) [64]. The second is those based on scattering of particles with a mass, such as electrons in scanning transmission electron beam microscopy (STEM) [65]. The third class are those based on an optical response such as confocal microscopy [66]. Each of these classes has its advantages and disadvantages with respect to chemical selectivity and spatial resolution. With STM the spatial resolution is extremely high, on the order of the atomic scale [67] and STEM approaches this as well [68]. However, these techniques are limited in their chemical specificity [69]. Furthermore, the scanning probe techniques are limited to surface studies of the sample, and the high vacuum needed for SEM causes liquid biological samples to dehydrate. 
A combination of optical spectroscopic techniques, such as Raman spectroscopy, vibrational spectroscopy, fluorescence spectroscopy and CARS, with microscopy offers advantages over AFM or SEM microscopy. The interaction of incident light with the sample is usually extremely weak, which is an advantage (over SEM) for non-destructive imaging, specifically for biological samples. Furthermore, optical imaging can image an area in-situ and real time where AFM uses a scanning tip to form a 2D image, where the scanning speed limits the image frame rate. However, the spatial resolution of optical techniques is usually limited by diffraction, which, for visible light, is 3 orders of magnitude larger than the atomic scale.

\section{Light-matter interaction}

The ability of optical detection to provide high chemical specificity can be understood as follows. The very complex dynamics of the electro-magnetic binding forces of electrons to the cores of atoms, as well as the binding forces between different atoms within a molecule or crystal structure, form the basis of the chemical properties of the material. Due to the nature of light, being the carrier of the electro-magnetic force, these binding forces can be probed by light beams, which, after detection of the response, reveal the response function of the sample as a function of the light beam properties. For instance, by tuning the light field frequency, a frequency dependent response can be obtained, showing characteristic absorption or emission frequencies and thereby reveal the chemical (atomic, molecular, crystalline, etc.) identity of the material. Besides simple optical absorption and emission (fluorescence) measurements a large variety of other optical techniques can be used, such as pump-probe experiments and optical nonlinear techniques. Provided that the resolution limit can be surpassed, it is this large variety of optical techniques and the associated large differentiation with respect to the chemical composition, which makes optical microscopy the most attractive candidate for imaging.

The abundance of energetic states of molecules stems from a set of types of excitation. These consist of electronic excitation (of electrons) and vibrational and rotational excitation of sets of atoms within the molecule. Each of these excitation regimes correspond to typical wavelength ranges. The range of vibrational transitions typically lies in the infrared region of the spectrum, rotational transitions typically lie in the range of terahertz and microwave radiation and the electronic transitions lie in the range of the visible to ultra violet spectrum [70]. Before excitation of the molecule with light, the molecule is likely to reside in one of the energetic ground states, since the electronic excited states have a large decay rate (of the order of ns or much faster). Furthermore, the chance of occupation of a vibrational or rotational excited level increases with the temperature of the molecule. A light field can excite the molecule from the ground state to one of the excited states provided that this transition is allowed, as governed by the so-called selection rules [70]. 


\section{CARS spectroscopy}

The CARS process is a nonlinear optical four-wave mixing process where three light fields, usually named the pump, Stokes and probe fields, induce the emission of a fourth light field (CARS field), indicated in fig. 4.1. It can be seen that the pump and Stokes beams are tuned such that their energy difference is resonant with a vibrational state of the medium which is also called twophoton resonant or Raman resonant. The purpose of such tuning to resonance is that this enhances the CARS output signal and allows to specifically address specific types of molecules. Through the two-photon resonant excitation of the sample with the pump and Stokes beam a coherence between the ground state and the vibrational state is created, which we call the vibrational coherence. A probe beam, incident on the sample in which such vibrational coherence is present, is scattered by the sample while the vibration of the molecules renders an amplitude modulation of the scattered light. Spectrally, this shows up as frequency sidebands that are separated from the original probe frequency and split by the frequency of the vibrational coherence. The upper sideband is called the CARS emission, the lower sideband is called the coherent Stokes Raman scattering (CSRS) emission.

The depth of the amplitude modulation and thus the strength of the CARS emission is proportional to the amplitude of the vibrational coherence which is enhanced significantly by the two-photon resonance condition, which is the exact tuning of the pump and Stokes frequency difference to the vibrational level. Therefore, by tuning the frequency difference between the pump and Stokes laser and observing the changes in the CARS intensity, the presence of some molecule specific vibrational levels is revealed. However, as a result of the selection rules for the transition between energetic states as induced by the set of light fields [71] not all vibrational levels can be probed using this CARS process. Nevertheless, by comparing the obtained CARS spectrum with a set of known spectra from chemicals, the constituents of the sample can be determined. This is why CARS or other vibrational spectroscopy techniques are often called chemically selective and a corresponding imaging is called chemical imaging.

For CARS, the nonlinearly induced polarization, when derived in its most simple approach with perturbation theory, is of third order and proportional to the applied fields $E$ as

$$
P^{(3)}(\omega) \propto \chi^{(3)} E\left(\omega_{p}\right) E\left(\omega_{S}\right) E\left(\omega_{p r}\right)
$$

Furthermore, the intensity of the CARS emission is proportional to the applied intensities as

$$
I_{\text {cars }} \propto \chi^{(3)} I_{p} I_{S} I_{p r}
$$

Since the value of $\chi^{(3)}$ is generally very small, light with high intensity such as can be generated with pulsed lasers are needed to generate a measurable CARS emission. 
The build-up of CARS emission intensity in the forward propagating direction (along the direction of the applied light fields) is described by [71]:

$$
I_{\text {cars }}(z) \propto z^{2} \operatorname{sinc}^{2}\left(\frac{\Delta k z}{2}\right)
$$

where $\Delta k$ is the wave vector mismatch $\left(\Delta k=k_{p}+k_{p r}-k_{S}-k_{\text {cars }}\right.$ with $k$ the wavevectors of the light fields) and $z$ the interaction length. As can be seen from this equation, the conversion efficiency along the propagation path is enhanced for the condition $\Delta k=0$ which is called the phase matching condition.

Actually, $\chi^{(3)}$ in eq. 4.1 is a tensor and the electric fields and the polarization are vectors, However, such vector equation can be reduced to a scalar equation once the geometrical orientation of the optical axes of the sample and the geometry of the incident light fields (the direction of propagation and the angle of polarization) are given. Therefore, the CARS emission conversion efficiency is a function of the polarization angle of the light (recall section 2.1.4 where a similar polarization angle dependence was described for the SHG process).

In our model of the CARS process we assume that the polarization angles of the light fields and their directions of propagation are such to maximize the CARS emission efficiency. Furthermore, the model is used to study the resolution improvement of certain CARS excitation schemes and therefore only CARS process which originate from small interaction volumes are considered. Therefore, the phasematching considerations and the development of the CARS emission intensity as a function of propagation distance (eq. 4.3) do not play a role.

\section{Numerical modeling of optical nonlinear processes}

As a tool to find and validate routes to obtain CARS spectroscopy images with a sub-diffraction limited resolution, we model the CARS process using the density matrix approach (see also, e.g., the work by Serrat et al. [72]). From the polarizations described by the off-diagonal elements of the density matrix, the field as radiated by the molecule can be determined as will be shown in more detain in section 4.2.2, while the diagonal elements display any population changes that may occur during CARS emission. Such a detailed description of the molecule, not only via its polarization in a perturbation approach (eq. 4.1) but also via the corresponding flow of populations, is chosen for the following reasons. Given a specific molecule (with specific energy levels and transition strengths) excited by a set of specific optical pulses (with a given spectrum and pulse duration), the exact response of the molecule to the optical pulses depends on the occupancy of the energetic levels and on the polarization between those levels. Furthermore, the optical pulses may lead to significant changes of the occupancy of the levels and thus the polarizations. The density matrix formalism is capable to adequately model such interactions with the light field whenever the influence of population changes due to the applied light field cannot be neglected. 
Other numerical techniques to study the CARS process have been presented in literature using a constant effective nonlinear coefficient to describe CARS generation, such as by Cheng et al. [73] to study CARS radiation beyond the paraxial approximation, or by Stankov et al. [74] and Wang et al. [75] to describe CARS propagation using a model based on coupled wave equations. However, also these models do not include the influence of the applied light fields to the effective nonlinear coefficient and are therefore limited in their applicability to explore novel approaches in controlling CARS emission.

In this chapter, we first present a density matrix model and results from an unmodified (standard) CARS process, in order to verify that the model accurately replicates known physical properties of CARS. After this verification we investigate a first deviation from standard CARS, which is a pulse sequencing scheme to increase CARS emission efficiency. This scheme might be an advantage in CARS experiments where the signal to noise ratio would normally be too low. In chapter 5 , the density matrix model will be used to study the effectiveness of a set of different routes to combine CARS with sub-diffraction limited imaging.

\subsection{Theoretical framework}

\subsubsection{Level scheme}

The standard description of CARS emission from molecules through a fourwave mixing process contains three levels of a molecular system (see fig. 4.1). In this description of the CARS process, the medium is illuminated by a set of three pulsed light fields with frequencies $\omega_{p}, \omega_{S}, \omega_{p r}$, called the pump, Stokes and probe fields, respectively. All fields are far detuned from the $|1\rangle-|3\rangle$ and $|2\rangle$ - $|3\rangle$ transition frequencies. However, to let the excitation of the sample become spectrally selective for vibrational transitions, providing chemical specificity, the pump and Stokes light fields are tuned such that there is a two-photon resonance between levels $|1\rangle$ and $|2\rangle$, through which $|2\rangle$ is populated. The population difference between the states $|1\rangle$ and $|2\rangle$ in combination with the pump and Stokes fields builds up a coherence between $|1\rangle$ and $|2\rangle$, called the vibrational coherence. This coherence then induces two optical sidebands on the probe light frequency, one of which is the CARS emission frequency $\left(\omega_{\text {cars }}=\right.$ $\left.\omega_{p}-\omega_{S}+\omega_{p r}\right)$. The other side band is called the coherent Stokes Raman scattering (CSRS) with an emission frequency $\left(\omega_{c s r s}=\omega_{S}-\omega_{p}+\omega_{p r}\right)$. In CARS experiments, the light fields $\omega_{p}$ and $\omega_{p r}$ are often chosen to be degenerate in frequency in order to simplify the experimental setup. However, for clarity and generality we use two different light frequencies for $\omega_{p}$ and $\omega_{p r}$ in our model.

\subsubsection{Spectrum and emission peaks}

In order to provide a theoretical prediction of the CARS emission amplitude, intensity or efficiency, a transformation between the results from the evaluated 


\section{$|3\rangle$}

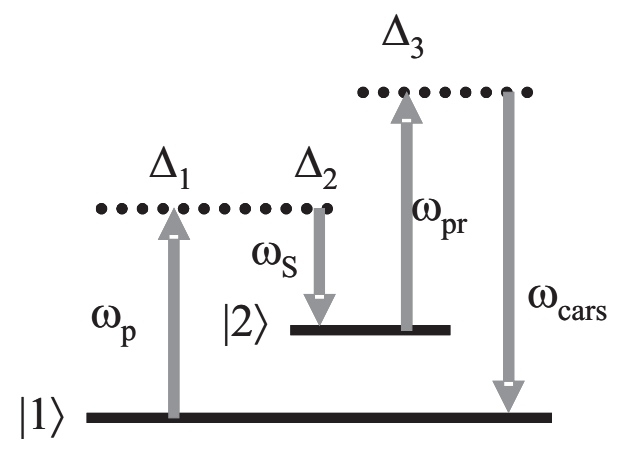

Figure 4.1: Three level system with a ground state $(|1\rangle)$ a vibrational state $(|2\rangle)$ and an excited state $(|3\rangle)$. The system has a dipole transition between states $|1\rangle$ and $|3\rangle$ and between $|2\rangle$ and $|3\rangle$. Three applied light fields pump $\left(\omega_{p}\right)$, Stokes $\left(\omega_{S}\right)$ and probe $\left(\omega_{p r}\right)$ are far detuned from the transition frequencies and polarize through a four-wave-mixing process the system such that a CARS $\left(\omega_{\text {cars }}\right)$ light field is radiated by the medium.

density matrix equations and the CARS intensity needs to be formulated.

We use the wave equation 2.30 that describes the relation between the radiative coherence terms $\rho_{i j}$ of the density matrix equations and the electric field $E$ in space. Recall that the radiative coherence terms as obtained from the evaluation of the density matrix equations are described with an effective carrier frequency equal to the detuning $\Delta$. In order to obtain the coherence evolution in a non-rotating frame, each coherence term is multiplied by a term oscillating with the transition frequency, e.g., $\rho_{13}(t) e^{i \omega_{13} t}$, thus creating terms that oscillate with $\omega_{13}-\Delta$.

In order to study the spectral content of the $E(t)$-field a Fourier transform is performed providing $E(\omega)$. The amplitude of the radiated electric field $E_{\omega}$ at frequency $\omega$ is obtained by integration of the wave equation (eq. 2.30) over space by assuming the source of radiation to be a point source:

$$
E_{\omega}=\frac{N k_{\omega} \mu}{\epsilon_{0}} \rho_{\omega} \int_{-\infty}^{\infty} \delta_{0}(z) d z
$$

where $\delta_{0}(z)$ is the Dirac delta function. To obtain from this the spectral representation of the emission intensity we use the standard expression

$$
I(\omega)=\frac{1}{2} c \epsilon_{0} n E(\omega) E^{*}(\omega)
$$

where $c$ is the speed of light in vacuum and $n$ the refractive index of the medium. For a typical calculation of the predicted emission spectrum is shown (fig. 4.2) 
when three strong light fields are applied while maintaining two-photon resonance (as illustrated in fig. 4.1). This spectrum looks rather complex at a first view, however a closer inspection of the emission frequencies reveals that each of the peaks corresponds to a particular optical process expected to occur. The strongest emission peaks correspond to Rayleigh scattering of the applied light fields $\omega_{p}, \omega_{S}, \omega_{p r}$ and the induced four-wave-mixing frequencies $\omega_{\text {cars }}$ and $\omega_{\text {csrs }}$. Furthermore, two peaks at the frequencies $2 \omega_{p}-\omega_{S}$ (labeled II) and $2 \omega_{S}-\omega_{p}$ (labeled I) are present. The calculated spectra, because they were numerically derived, consist of discrete data points. To derive, in spite of this, reliably the peak intensity values of the various emission processes, we used fit functions on each single peak.

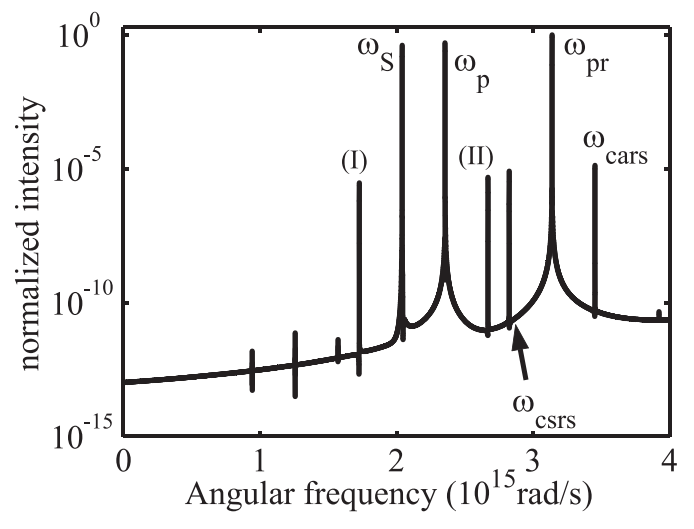

Figure 4.2: Example of a calculated emission spectrum from a three-level medium (see fig. 4.1) to which three light fields are applied. The peak features are the linear Rayleigh scattering of $\omega_{S}, \omega_{p}$ and $\omega_{p r}$. The four-wave mixing processes of Coherent-Stokes-Raman scattering $\omega_{\text {csrs }}$ and Coherent Anti-Stokes Raman Scattering $\omega_{\text {cars }}$. Furthermore, the Stokes shifted field of $\omega_{S}(\mathrm{I})$, and the anti-Stokes shifted field of $\omega_{p}$ (II) are present.

To define an emission efficiency, $\eta_{\text {cars }}$, we insert into eq. 4.4 using $\chi_{\text {in }}=$ $\frac{E_{i n} \mu}{\hbar}$, where $E_{i n}=\sqrt{E_{p}^{2}+E_{S}^{2}+E_{p r}^{2}}$, and obtain

$$
\eta_{\text {cars }}=\frac{I_{\text {cars }}}{I_{\text {in }}}=\left(N \mu^{2} \frac{k_{\text {cars }}}{\epsilon_{0} \hbar} \frac{\rho_{\text {cars }}}{\chi_{\text {in }}}\right)^{2}
$$

where $\rho_{\text {cars }}$ is the amplitude of the frequency component of $\rho(\omega)$ at the CARS 
frequency and $\chi_{i n}$ is the sum of the applied fields expressed in terms of Rabifrequency $\chi_{p}, \chi_{S}$ and $\chi_{p r}$.

\subsubsection{Numerical model}

In the remainder of this chapter, the model based on the density matrix equations for a $\Lambda$-type system (recall section 2.2.4) is used to study the relations between $\rho_{\text {cars }}$, and the intensity of the applied fields. The relation between $\rho_{\text {cars }}$ and the two-photon detuning of the pump and Stokes fields with respect to the vibrational level are studied in section 4.2.4. Subsequently, in section 4.3, the effect of a mutual pulse delay between the pump, Stokes and probe beams on the CARS emission efficiency is studied, to find the pulse sequencing scheme that provides the highest conversion efficiency. In chapter 5 , this model is used to study the effectiveness of a set of schemes which are designed to suppress the CARS emission efficiency in order to find methods to combine sub-diffractionlimited imaging techniques with CARS spectroscopy.

For this $\Lambda$-type system we choose energy levels, light field carrier frequencies and pulse durations that are typical for CARS emission processes from molecules. Specifically, we do not choose values for a particular molecule so that our results remain general. In view of the complexity of the system, we use numerical solutions (fourth-order Runge-Kutta algorithm with a fixed step size) to solve eq. 2.31. The $|1\rangle-|3\rangle$ transition angular frequency is set to $4.7 \cdot 10^{15} \mathrm{rad} / \mathrm{s}\left(\approx 400 \mathrm{~nm}, \approx 25,000 \mathrm{~cm}^{-1}\right)$, and the $|1\rangle-|2\rangle$ angular frequency to $314 \cdot 10^{12} \mathrm{rad} / \mathrm{s}\left(\approx 6 \mu \mathrm{m}\right.$ or $\left.\approx 1666 \mathrm{~cm}^{-1}\right)$. As typical wavelengths of the light fields $\lambda_{p}=800 \mathrm{~nm}, \lambda_{S}=923 \mathrm{~nm}$ and $\lambda_{p r}=600 \mathrm{~nm}$ are chosen, such that the pump and Stokes fields are two photon resonant with the $|1\rangle-|2\rangle$ transition. The total lifetime $\left(\frac{1}{R_{31}+R_{32}}\right)$ of state $|3\rangle$ is taken to be of the order of nanoseconds [76], while the decoherence rates between states is of the order of picoseconds (see e.g., [76-78]). Typical light pulse durations $\tau$ are set to a few picoseconds $\left(1 / e^{2}\right)$. The simulations extend over $30 \mathrm{ps}$ in steps of $0.1 \mathrm{fs}$.

\subsubsection{Tests of the numerical model}

As an initial test to verify that the model replicates experimentally observed characteristics of the CARS process, two types of numerical experiments are performed.

\section{Intensity dependence of CARS emission}

The efficiency $\eta$ of a four-wave mixing process like CARS scales with respect to the applied intensity $I^{3}(\omega)[43]$. Therefore, using eq. 4.6 a relationship between the CARS emission and the applied fields of the following form is expected:

$$
\rho_{\text {cars }}^{2 / 3}=C \chi_{\text {in }}^{3}
$$

where $C$ is a constant. In order to test the model with respect to this intensity dependence, a set of calculations was performed in which $\chi_{i n}$ was varied in 
amplitude. The amplitude of the coherence $\rho_{\text {cars }}$ at $\omega_{\text {cars }}$ for each calculation was calculated.

The results from this set of calculations is shown in fig. 4.3. In this graph the third root of $\rho_{\text {cars }}^{2}$ is plotted versus $\chi_{\text {in }}^{2}$ and it can be seen that the results closely follow a proportionality except for the highest values of $\chi_{i n}^{2}$. The proportionality indicates that the CARS intensity predicted by our density matrix model indeed scales as the third power of $I$ for low values of $I$, in agreement with theory (see eq. 4.2). For high intensities, the model predicts a deviation from $I^{3}$ due to the saturation of the $\rho_{12}$ density matrix element (which describes the coherence between the ground state and the vibrational level). This saturation would normally be accompanied by higher order nonlinear optical processes, however, here we are not interested in those processes and rather intend to study more basic properties of CARS emission. Therefore, in all following studies, a maximum value of the induced Rabi frequency $(\chi)$ of $2 \cdot 10^{13} \mathrm{rad} / \mathrm{s}$ of the pump, Stokes and probe fields, is used.

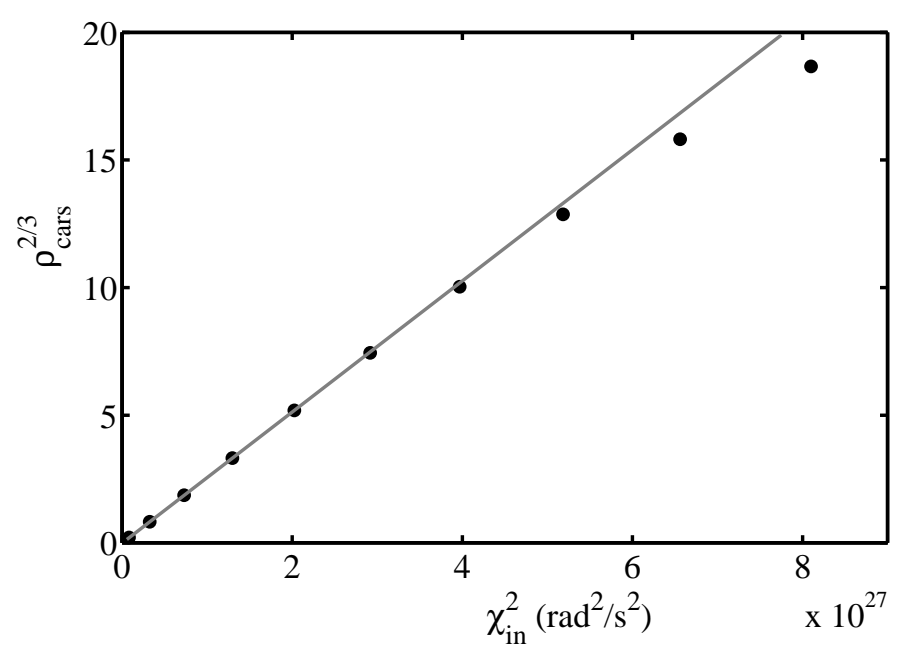

Figure 4.3: Calculated CARS intensity (black dots) expressed in terms of $\rho_{\text {cars }}^{2}$ as a function of summed applied intensity of the pump, Stokes and Probe pulses expressed in terms of $\chi^{2}$. The gray line indicates the best fit for the lower set of values of $\chi_{i n}$.

\section{Two-photon-resonance dependence}

As a second test, the CARS emission intensity as a function of the two-photonresonance detuning is calculated. According to standard theory, the two photon resonance $\left(\omega_{p}-\omega_{S}=\omega_{12}\right)$ enhances the efficiency of the build-up of coherence $\rho_{12}$ which, in turn, enhances the CARS emission intensity [79]. In a set of 
calculations we varied the two-photon detuning by varying $\omega_{S}$ and keeping $\omega_{p}$ and $\omega_{p r}$ a constant. The CARS emission calculated for various two-photon detunings is shown in fig. 4.4. In this graph the CARS efficiency with respect to the two-photon resonance detuning shows a sharp enhancement of the CARS emission at the two-photon resonance according to theory.

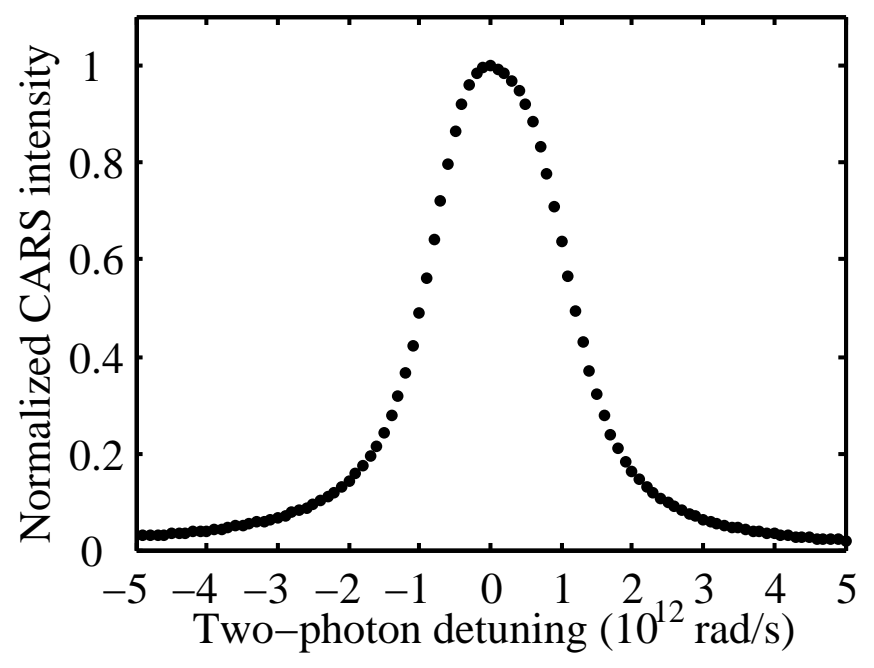

Figure 4.4: CARS intensity calculated with a constant $\omega_{p}$ by tuning $\omega_{S}$ across two-photon resonance with the vibrational level, normalized to the intensity at zero detuning (the dotted line serves as a guide to the eye). The sharp increase of the CARS emission at zero detuning (resonance) is a characteristic feature of the CARS fourwave mixing process.

\subsection{Enhancement of CARS emission efficiency}

In a practical experimental setup of CARS microscopy, the CARS emission intensity for some vibrational features may be too small to detect, limiting the ability to discriminate between different materials in an image. To increase the signal to noise ratio, several approaches, such as pulse shaping and phase sensitive detection have been reported [80,81]. Through pulse shaping, where a $\pi$ phase step was introduced in the spectrum, the ratio between the resonant CARS emission and the non-resonant part was increased producing an improved signal to noise ratio [80]. Since CARS is a parametric process the resonant CARS emission is related to the phase of the applied light fields. Through phase sensitive detection the non-resonant background can be subtracted from the measured CARS intensity [81]. 
In order to search for additional ways to not only suppress a background but to increase the CARS signal, we investigated the influence of a small delay between the drive pulses. With the three level model as described in section 4.2 a set of calculations was performed to investigate pulse sequencing strategies. For a simultaneously single-photon and two-photon resonant excitation, Sautenkov et al. have shown experimentally that a non-zero delay between the pump and Stokes pulse increases the vibrational coherence which enhances the CARS emission efficiency [82]. This was also validated by a numerical model based on the density matrix equations. However, their choice of the additional single-photon resonance may be problematic if it causes an easier photodamage. Furthermore, only the influence of a pulse delay between the pump and Stokes fields was considered. In this section, we explain, in terms of the noninstantaneous response of the medium, how pump and Stokes pulse sequencing lead to enhanced CARS emission also in the domain of main importance, where the light fields are far off-resonant with the single-photon transitions. Furthermore, an additional enhancement of the CARS emission is found by delaying the probe beam with respect to the pump and Stokes beams.

As detailed earlier, the CARS emission is the result of amplitude modulation of the probe beam. Therefore, CARS emission is maximized when the depth of modulation of the probe beam is maximized. The modulation itself is due to the vibrational coherence $\rho_{12}$, so maximizing the efficiency of CARS involves maximizing this vibrational coherence. A promising approach lies in the variation of the pulse sequence and timing of the pump, Stokes and probe pulses. For this purpose, the idea of Sautenkov et al. was to increase the CARS signal with a technique that is known as Stimulated Raman Adiabatic Passage (STIRAP).

It is well known from theoretical and experimental work on STIRAP $[83,84]$ that the efficiency of coherent population transfer in three level media depends on the pulse sequence and temporal delay of the pulses. In those types of experiments in $\Lambda$-type media, it was found that the population, initially in one ground state, was transferred with an increased efficiency to the other ground state by a set of pulses that are delayed with respect to one another in what is called a counter-intuitive timing, due to a build-up of vibrational coherence. Since the CARS emission efficiency depends on the build-up of vibrational coherence as well, a similar counter-intuitive delay between the pump and Stokes pulse increases the CARS emission.

We explain the maximization of the vibrational coherence by the counterintuitive delay between the pump and the Stokes (the Stokes pulse advances the pump pulse) as follows: We here consider the case that the medium is initially in a state with all of the population in the lowest ground state and two pulses, pump and Stokes are applied to the medium. We divide the dynamical response into two periods in time. During the first period, the pump field and the population difference $\left(\rho_{33}-\rho_{11}\right)$ drives the coherence $\rho_{13}$ which, in turn, drives the population to state $|3\rangle$. Note that during this period $\rho_{13} \gg \rho_{23}$. During the second period, the Stokes field and the population difference $\left(\rho_{33}-\rho_{22}\right)$ drives the co- 
herence $\rho_{23}$ and $\rho_{23} \gg \rho_{13}$. Furthermore, the build-up of vibrational coherence is described by the density matrix equation $\dot{\rho}_{12}(z, t)=\frac{i}{2}\left(\chi_{13}^{*} \rho_{23}^{*}-\chi_{23} \rho_{13}\right)$. In this equation it can be seen, that a maximal build-up of vibrational coherence is expected when $\chi_{23}$ and $\rho_{13}$ reach a maximum simultaneously, e.g., the amplitude of the Stokes pulse is maximal during the first period of the excitation scheme. Similarly, a maximal vibrational coherence is expected when $\chi_{13}$ and $\rho_{23}$ reach a maximum simultaneously, e.g., the amplitude of the pump pulse needs to reach its maximum during the second period of the excitation scheme.

In the next sections, the results of calculations are reported where the pulse sequence and delay of the pump, Stokes and probe pulses are varied to observe whether the suggested counter-intuitive pulse delay would indeed increase the CARS emission efficiency.

\subsubsection{Stokes pulse delay}

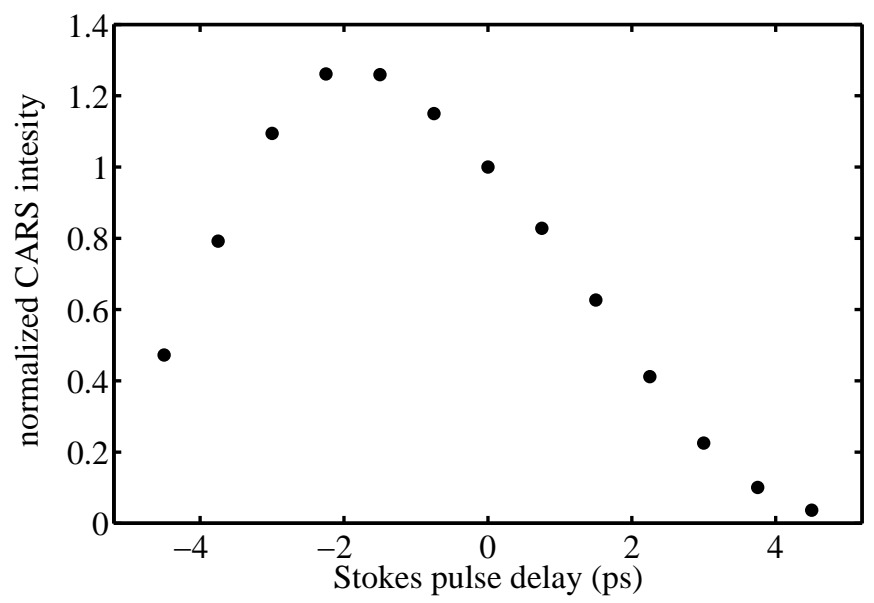

Figure 4.5: CARS intensity as a function of Stokes pulse delay normalized to the intensity at zero delay. The CARS emission efficiency is maximized by the advancement of a Stokes pulse by 2 ps. The maximum increase in CARS emission efficiency is about $25 \%$.

The results of the calculations for the CARS emission intensity as a function of the delay of the Stokes pulse with respect to the simultaneous pump and probe pulses is shown in fig. 4.5. Here the pulse delay of the Stokes pulse is varied between -5 ps and +5 ps. The pulse durations of all pulses are set to 3 ps. It can be seen that in this set of calculations the maximum efficiency is obtained when the Stokes pulse arrives 2 ps in advance of the pump and probe pulses. The increase in efficiency with respect to zero delay is about $25 \%$. The cause of the increase can be understood as follows. The delay of 
the pump pulse with respect to the Stokes pulses results in a larger growth of the vibrational coherence, similar to the STIRAP process as described above. Since the CARS emission is a result of the modulation of probe beam with this vibrational coherence, the increase in vibrational coherence increases the CARS emission as well.

\subsubsection{Probe pulse delay}

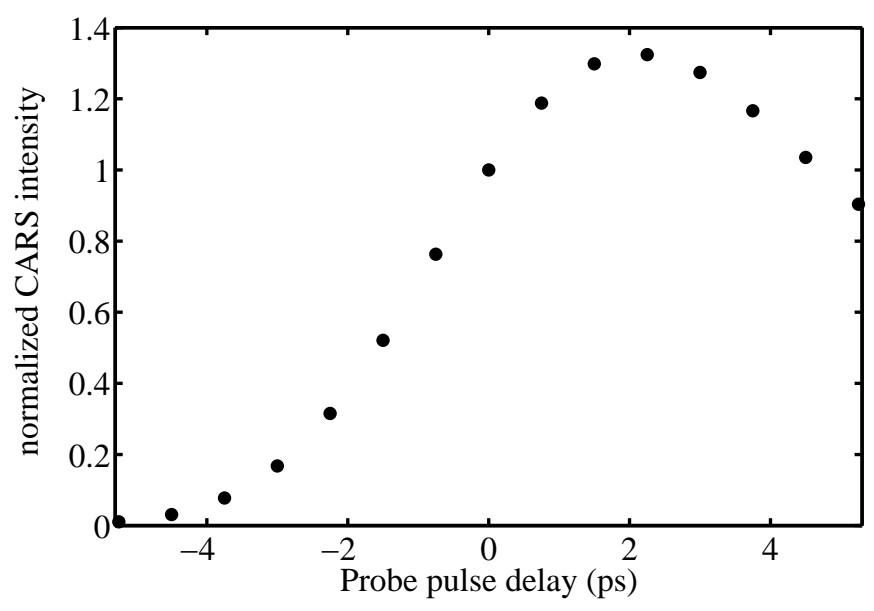

Figure 4.6: CARS intensity as a function of probe pulse delay normalized to the intensity at zero delay. The CARS emission efficiency is maximized by the delay of the probe pulse by $2 \mathrm{ps}$. The maximum increase in CARS emission efficiency is $32 \%$.

The vibrational coherence, which induces the CARS emission when probed with the probe beam, grows in time due to the pump and Stokes beams and decays due to decoherence effects, i.e., mainly via the damping constant $\beta_{12}$ (in eq. 2.31). Therefore, the maximum vibrational coherence is expected to occur just after the pump and Stokes beams have reached their maximum intensity. To investigate this expectation we used a pulse sequencing such as in a pumpprobe experiment, where the probe pulse is delayed with respect to the pump pulse. In order simultaneously to maximize the CARS emission efficiency as described in section 4.3.1, the Stokes pulse was set at a delay of -2 ps with respect to the pump pulse. The probe pulse delay with respect to the pump pulse was varied between $-5 \mathrm{ps}$ and $+5 \mathrm{ps}$. The pulse durations of all pulses are set to 3 ps. As can be seen in fig. 4.6 where the results of the calculations are displayed, the maximum efficiency is obtained when the probe pulses is delayed with respect to the pump pulse by 2 ps. The increase in efficiency with respect to zero delay is $32 \%$. 
By combining the advancement of the Stokes pulse and delaying the probe pulse with respect to the pump pulse a total increase of CARS emission intensity of $66 \%$ with respect to zero delay was obtained. It should be noted, however, that this amount of enhancement was only calculated for one specific set of parameters (as specified in section 4.2.3), and may therefore vary for different values for the decoherence rates and excitation field strengths. The result where the Stokes beam advances the pump beam show a qualitative agreement with experimental results as obtained, by Sautenkov et al. [82]. A quantitative comparison could not be performed since their report did not provide sufficient amount of data. Note further that Sautenkov et al. did not vary the delay between the pump and probe in their calculations or experiments, therefore, a direct comparison between our results of where the probe is delayed with respect to the Stokes and pump could not be made.

\subsection{Conclusion}

In this chapter, a numerical model based on the density matrix equations to describe the CARS emission process has been presented. It was shown that this model can be used to study the dynamics of the population densities of a three level system as well as the coherences and thus provides an extension well beyond the standard description of CARS based on the perturbative model with a $\chi^{(3)}$ nonlinear coefficient. The spectrum of emission was obtained from the temporal development of the coherences. Some well known features of CARS emission in the perturbative description have been modeled to verify our model. It was found that our three-level density matrix model was sufficiently detailed to reproduce all of the critical physical phenomena observed in real CARS experiments.

Furthermore, the possibility to increase the efficiency of CARS emission by the advancement of the Stokes pulse and delay of the probe pulse has been shown. In our model, the maximum increase in the efficiency for a CARS experiment with degenerate pump and probe pulses was found to be about $25 \%$. However, this result is expected to vary depending on the lifetimes and decoherence rates of the material. We also show that in the case where the pump and probe are not degenerate, the efficiency can be further increased by a factor of $32 \%$ by delaying the probe pulse by 2 ps. The total increase by both advancing the Stokes pulse and delaying the probe pulse with respect to the pump pulse is $66 \%$. We note that this could be tested using pulse shaping techniques to create a pump field with two peaks with a delay between them. 


\section{Sub-diffraction-limited resolution CARS}

To introduce sub-diffraction-limited resolution to CARS in full analogy to STED, a saturation process similar to STED would have to be identified. However, this appears to be difficult because the physical process of CARS does not involve any population inversion. From this we concluded that a more promising approach lies in manipulating the coherence between the ground state and the vibrational state (the so-called vibrational coherence), the presence of which is necessary for CARS emission. The analogy between CARS and STED would lie within a nonlinear process that reduces the vibrational coherence in a saturable process. In the following we will work out what this can mean.

This chapter is ordered as follows: First a general overview of currently known methods to obtain an image with a sub-diffraction-limited resolution is given in order to point out suitable routes to integrate such methods with CARS microscopy. The limits in effectiveness of a method based on interference with an additional beam with a carrier frequency equal to that of the CARS emission as proposed by Nikolaenko et al. [85] is studied and discussed in section 5.2. For an improvement beyond this, we first propose a novel scheme to suppress the emission of CARS by preparing the medium prior to the arrival of the pump, Stokes and probe beams such that the vibrational level becomes occupied equally as compared to the ground state. The effectiveness of this scheme and the explanation why such suppression would improve the spatial resolution and also which improvement factors seem possible is discussed in section 5.3. In section 5.4 we propose a second, alternative approach for resolution improvement in CARS microscopy. The alternative approach is not 
based on suppression, but instead uses an additional control beam to induce Rabi oscillations with a spatially dependent frequency. These Rabi oscillations periodically alter the ground state population and vibrational coherence of the system which modifies the CARS emission efficiency periodically. It will be shown that this modulation induces sidebands to the CARS emission line in the spectrum, and that the splitting of these sidebands indicates the position of an emitter with respect to the center of the control beam. We show that, by analyzing such a modified spectrum, an improvement in resolution can be achieved, for which we name potential improvement factors as well.

\subsection{Overview of schemes for improvement of spatial resolution}

The transverse resolution in a conventional optical imaging system using lenses, is limited by the diffraction limit. This fundamental limit occurs when only propagating waves that are radiated by an object are collected by the lens system. To illustrate this, we consider a monochromatic plane wave $E$ propagating in space with a refractive index $n$ which can be written as $E=E_{0} e^{i\left(\mathbf{k} x-\frac{n 2 \pi c t}{\lambda}\right)}$ where $\lambda$ and $c$ are the wavelength and speed of the light in vacuum, respectively, and $\mathbf{k}$ is the wave vector. For a plane wave satisfying Maxwell's equations, the wave vector must satisfy $\mathbf{k}^{2}=n^{2} k_{0}^{2}$, with $k_{0}=2 \pi / \lambda$. Light fields with a real valued wavevector propagate as radiation whereas the amplitude of light fields with imaginary valued wavevectors decay exponentially with the distance in the propagation direction, which is known as the evanescent field. The propagating plane waves are thus characterized by wavevectors such that $|\mathbf{k}| \leq n k_{0}$. As a result of the large difference between the very large decay in amplitude of the evanescent waves as compared to the very small decay in amplitude of the propagating waves (in free space) only the propagating waves are imaged by a lens system. The image, formed by the lens, only contains spatial features with $\mathbf{k}$-vectors contained in the propagating light field. Since the range of k-vectors is limited, the resolution is limited as well, this is known as the diffraction limit.

There are, however, several useful approaches to overcome this diffraction limit. For instance, the nonlinear effect of frequency conversion by the material can be used since the nonlinearly scattered light, e.g., second harmonic generated light, contains a larger range of $\mathbf{k}$-vectors as will be further expanded upon in section 5.1.1. Another approach is to illuminate the sample, which, through a nonlinear interaction mechanism, excites the medium with a spatial pattern which contains a large k-vector. Such a nonlinear interaction is found, for instance, in the process of depletion, which will be discussed in more detail in section 5.1.2. And another type of nonlinear interaction, saturable suppression, can be implemented using a spatial pattern containing a large $\mathbf{k}$-vector as will be shown in section 5.3 where a CARS emission process is combined with such a saturable suppression process. Furthermore, in section 5.4 it will be shown using yet another type of nonlinear interaction based on a modula- 
tion by induced Rabi oscillations that the CARS emission from a sample can be spatially labeled. The increase in resolution is then obtained by accurately resolving the frequency splitting. The resolution of the frequency measurement can then be used to increase the range of $\mathbf{k}$-vectors in the image.

\subsubsection{Reduced excitation area by nonlinear interaction}

A weak light field, propagating through a material that does not noticeably absorb light, causes a small amount of electronic charge displacement called the induced polarization, which is oriented opposite to the externally imposed field. At low applied field strengths the resulting polarization and the field that caused it are proportional to the applied field, which means that diffraction limited spatial field distributions would excite a sample only in a diffraction limited manner as well. But, as was described in chapter 2 via eq. 2.1, when the light field is not too intense and in the case the absorption is negligible because the incident fields are not resonant with any transition, the polarization response can be approximated by a Taylor expansion in terms of the incident light field strength. The nonlinearity of the response can then lead to a spatially reduced excitation area.

In chapter 3 we described as a typical example of such nonlinear response the frequency doubling of an intense light field by a crystal with a non-centrosymmetric crystal structure [43], also known as second harmonic generation (SHG). Although the light fields required to observe such non-resonant optical effects are rather large [86], the conversion efficiency (of SHG for example) scales with the nonlinear order of the process (quadratically for SHG) with the intensity of the incident light beam. An improvement of the resolution can then be expected for the following reason. The area of a focused beam where the local intensity is sufficient to contribute to a detectable nonlinear response, reduces as the nonlinear order of the response increases. For the case of a transverse Gaussian beam this area $A$ scales as:

$$
A \propto \frac{1}{\sqrt{n}}
$$

with $n$ the order of optical response. Since the excitation area of a nonlinear response is smaller than for the case of the linear response (for which $n=1$ ), an imaging system that makes use of such a nonlinear response has a better resolution. The process under consideration here, CARS, is of the third nonlinear order, therefore an increase of resolution is expected, but this would only be a maximum factor of $\sqrt{3}$ with respect to Abbe's diffraction limit.

\subsubsection{Reduced excitation area by suppression}

Hell and co-workers realized that the saturation of a transition with resonant, intense light, which is a nonlinear response that could be described by increasingly high orders in the driving intensity, could be used to obtain subdiffraction-limited images $[12,13]$. Their technique of Stimulated Emission 
Depletion (STED) can be described using the level system of a fluorophore molecule shown in fig. 5.1. The technique is based on the competition of two relaxation processes from level $(|2\rangle)$ which is populated by a pump laser $\omega_{p}$. The decay from this level normally occurs through fluorescence $\omega_{f}$ (spontaneous emission) which scales proportionally to the population density of level $|2\rangle$ and provides the light field to be detected. However, if an additional and strong light beam with a frequency $\omega_{c}$ is injected, called the control beam, another competing route for depleting state $|2\rangle$ occurs through stimulated emission to lower state $|3\rangle$. The depletion of $|2\rangle$ extinguishes the fluorescence at $\omega_{f}$ in a saturation-type of process, i.e., in a nonlinear response to the intensity at $\omega_{c}$ : the amount of fluorescence at $\omega_{f}$ approaches zero when the intensity at $\omega_{c}$ is increased while the excitation by $\omega_{p}$ is held unchanged.

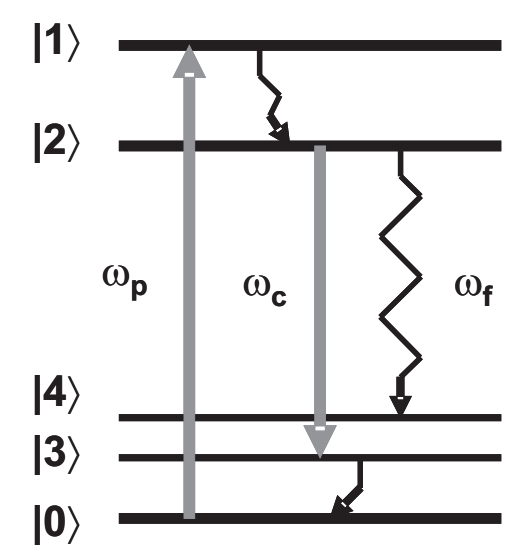

Figure 5.1: Level scheme for the STED process. The atom is excited into state $|1\rangle$ by a pump laser $\left(\omega_{p}\right)$ and relaxes to level $|2\rangle$. From this level, the medium may relax back to the ground state via two routes: stimulated emission by injecting a control beam with frequency $\omega_{c}$, or by fluorescence $\left(\omega_{f}\right)$. The fluorescence emission is suppressed by depleting the level $|2\rangle$ through stimulated emission with the control beam.

The basic idea to use the saturation-type of depletion of state $|2\rangle$ to achieve an increased spatial resolution can be expressed as follows. The pump beam is chosen as, e.g., a Gaussian TEM $_{00}$ mode with a bright spot in its center (see fig. 5.2) to illuminate a sample consisting fluorophores. The pump frequency is tuned so that the fluorophores are excited. Simultaneously, the control beam is chosen to possess a node or vortex, such as is present in a doughnut shaped profile (see fig. 5.2) co-propagating with the pump beam such that their centers are aligned. The control beam's frequency is chosen so that it depletes the fluorophore through the process of stimulated emission. Over the volume of fluorophores where the control beam intensity exceeds the saturation inten- 

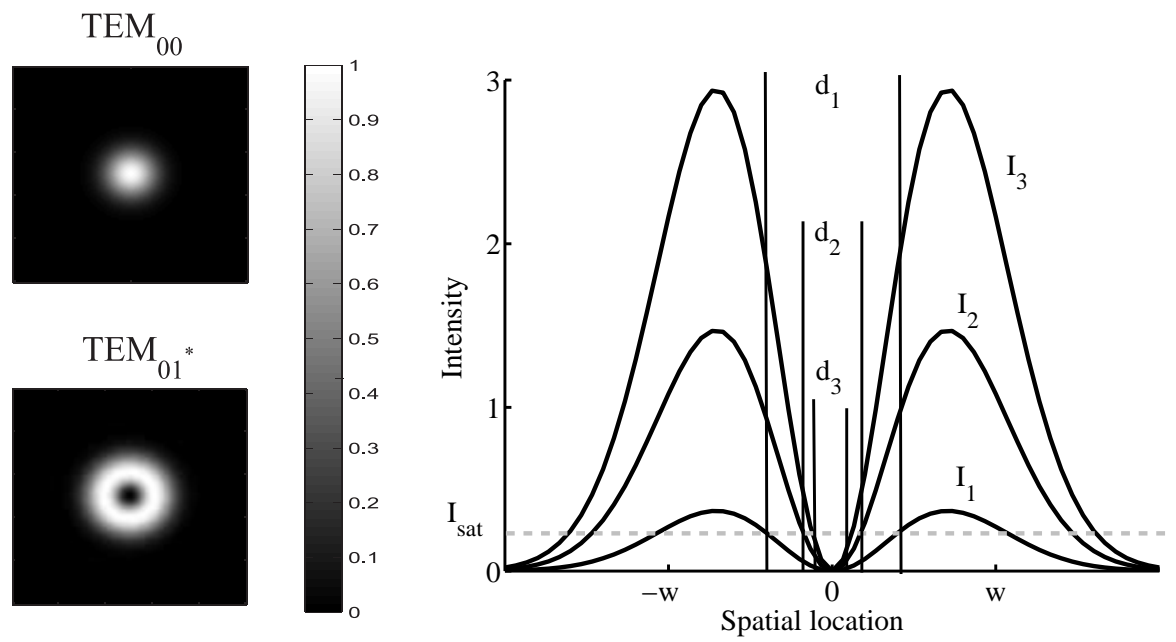

Figure 5.2: (left) Transverse intensity profile of a fundamental Gaussian mode $\left(\mathrm{TEM}_{00}\right)$ for excitation at $\omega_{p}$ and a doughnut-shaped mode $\left(\mathrm{TEM}_{01^{*}}\right)$ for depletion at $\omega_{c}$ such as can be obtained by a superposition of two $\mathrm{TEM}_{01}$ Laguerre-Gaussian modes. (right) Cross section of the intensity profile of doughnut shaped beams (with a beam waist $w)$ of increasing intensity $\left(I_{1}<I_{2}<I_{3}\right)$. It can be seen that the diameter around the nodes where $I_{c}<I_{s a t}$ decreases $\left(d_{1}>d_{2}>d_{3}\right)$ as the maximal intensity of the doughnut shaped beam increases.

sity of the fluorophore, stimulated emission dominates and the excited state population is quickly reduced to zero. The decay of the fluorophores in the volume where the control laser intensity is less than the saturation intensity, i.e., around the node of the beam, remains dominated by spontaneous emission, which takes place on a timescale that is associated with the lifetime of the excited state. As is illustrated in fig. 5.2 (right) and explained in the caption, the spatial range within which the intensity of the depleting doughnut-shaped control beam is below the saturation intensity becomes smaller with increasing peak intensity of the control beam. It is, therefore, possible to create a volume of excited fluorophores that is smaller than the diffraction limit using two diffraction-limited beams.

When looking in more detail, the non-depleted volume is inversely proportional to the control beam intensity, so, in principle, the resolution can be increased simply by increasing the control beam intensity.

The resolution $d r$ is approximately given by $d r \approx 0.45 \frac{\lambda}{n \sin \alpha \sqrt{1+I_{c} / I_{s a t}}}$ with $\sin \alpha$ the numerical aperture of the lens and $n$ the refractive index of the medium between the sample and the lens [87]. By scanning across the sample, a sub- 
diffraction-limited resolution image can be formed. Recently, using this technique, a resolution of $6 \mathrm{~nm}$ was achieved [17]. In order to investigate in how far the type of mechanisms and methods underlying the resolution improvement in STED can be translated to a potential improvement of resolution in CARS microscopy, we decided to look to more detail in the dynamics of the fluorophores in STED, but using the density matrix formalism that we have earlier presented for the description of CARS (chapter 4).

\section{Comparison of STED and CARS}

In STED where the strong control field is on-resonance with a transition of the medium, the dominant optical effect is stimulated emission or absorption of the light. The effect of the control laser might then be similar to the dynamics of Rabi-oscillations that we described in chapter 2. Actually, however, it turns out that the similarity is limited, which is caused by the relatively high damping on the control transition. With low damping, as was described in chapter 2, the light field drives the polarization to a large magnitude and the atom would fully reach the lower state. Once in the lower state, the control light would drive the anti-phased polarization of the atom until it fully reaches the excited state again. In a STED scheme, however, this oscillatory behavior is not observed due to a strong damping of the coherence such as by collisional dephasing or decay of the lower state which is not the ground state. More specifically, the decoherence rate is larger than the Rabi oscillation period such that the response of the fluorophores on the control laser is that of an overdamped system. The strong damping of the coherence inhibits that a noticeable induced polarization builds up. Such response is also called an incoherent response. In the density matrix equations, the off diagonal elements can be adiabatically eliminated [44], leaving only the dynamics of the diagonal elements, which are then commonly called the rate equations. For the closed system used to describe STED with five levels as shown in fig. 5.1, these are:

$$
\begin{aligned}
& \dot{\rho}_{00}=-\sigma \Phi_{p}\left(\rho_{00}-\rho_{11}\right)+A_{30} \rho_{33}+A_{40} \rho_{44} \\
& \dot{\rho}_{11}=\sigma \Phi_{p}\left(\rho_{00}-\rho_{11}\right)-A_{12} \rho_{11} \\
& \dot{\rho}_{22}=-\sigma \Phi_{c}\left(\rho_{22}-\rho_{33}\right)-A_{24} \rho_{22}+A_{12} \rho_{11} \\
& \dot{\rho}_{33}=\sigma \Phi_{c}\left(\rho_{22}-\rho_{33}\right)-A_{30} \rho_{33} \\
& \dot{\rho}_{44}=A_{24} \rho_{22}-A_{40} \rho_{44}
\end{aligned}
$$

Here, the coefficients $A_{j k}$ are the spontaneous emission rates from the state $|j\rangle$ to state $|k\rangle, \sigma$ the absorption cross section and $\Phi_{p}$ and $\Phi_{c}$ the photon flux of the pump beam and control beam respectively.

The simplest way to calculate the quantitative evolution of the diagonal elements of $\rho$, and thus also the functional shape of the fluorescence versus increasing control intensity in a STED process can be found by solving the system of equations, e.g., by assuming steady state as if the fluorophores were driven by continuous wave light fields. To provide an illustrative, typical example 


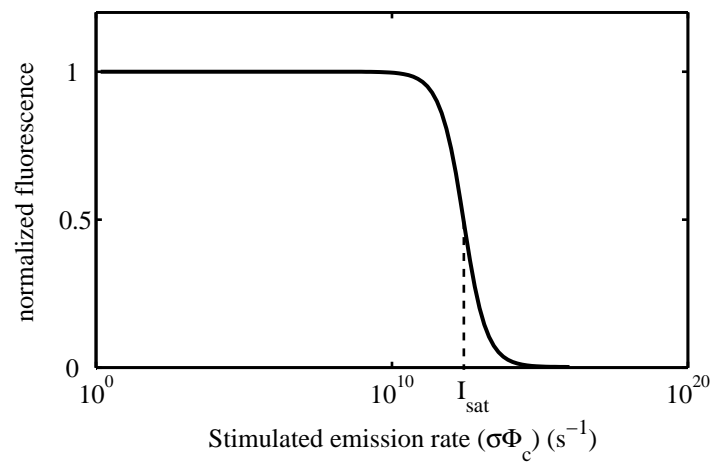

Figure 5.3: The total amount of spontaneous emission (fluorescence) as a function of the stimulated emission rate induced by the control beam in a STED process normalized to case without a control beam present. The curve follows a saturation curve (see eq. 5.7) and shows that the fluorescence emission is suppressed at high intensities of the control beam. The definition of the saturation intensity, the point at which the amount of fluorescence is half of the maximum value, is indicated in the graph as the dashed line.

also for the case typically used in STED experiments, we solved the equations numerically with typical parameters found in STED experiments [12]. For the case that the decay rates $A_{30}, A_{40}, A_{12}=10^{12} \mathrm{~s}^{-1}$ (a lifetime of $1 \mathrm{ps}$ ) and $A_{24}=10^{9} \mathrm{~s}^{-1}$ (a lifetime of $1 \mathrm{~ns}$ ) and the pump absorption rate $\sigma \Phi_{p}=50 \cdot 10^{9} \mathrm{~s}^{-1}$, the total spontaneous emission (the integral of $A_{24} \rho_{22}$ over time) as a function of the stimulated emission rate induced by the control beam $\left(\sigma \Phi_{c}\right)$ is calculated. The results of this calculation is shown in fig. 5.1.2, where it can be seen that the amount of spontaneous emission (fluorescence), $S$ normalized to the unsuppressed case, indeed follows the well-known saturation curve.

$$
S=\frac{1}{1+\frac{I_{c}}{I_{s a t}}}
$$

In this expression $I_{c}$ is the control beam intensity and we have defined $I_{s a t}$ as the intensity at which the fluorescence intensity is reduced to half of its maximum.

To make use of this saturation type of response of the fluorescence for increasing the spatial resolution in STED microscopy, a control beam with a node or vortex can be applied as was described above in fig. 5.2. An example of such a beam is a doughnut shaped beam which can be formed by a superposition of two Hermite-Gaussian modes, by a spiral phase plate in a TEM $_{00}$ beam or also by the linear combination of two instances of a $\mathrm{TEM}_{01}$ Laguerre-Gaussian 
mode, where one of the modes is rotated in plane by $\pi / 4$ with respect to the other.

The doughnut shaped beam is used to suppress fluorescence emission from all but a small volume at the center of the beam, provided that the intensity of the control beam exceeds the saturation intensity $\left(I_{\text {sat }}\right)$ over the entire area illuminated by the pump beam outside the node. By increasing the intensity of the control beam, the radius of the circle around the center of the beam where $I_{c}<I_{\text {sat }}$ can be reduced to values below the diffraction limit (see fig. 5.2 (right)).

From this section, we can conclude that a promising approach to add to CARS microscopy a sub-diffraction-limited imaging capability is to find a saturation type of suppression process similar to STED. However, it should be noted that the CARS process is based on a build-up of coherence between the ground state and a vibrational state (recall chapter 4) by using off-resonant light fields, which, compared to STED, is a very different process, since STED uses a suppression of the build-up of population by using light fields that are on-resonance. The aim for saturable suppression of CARS should therefore be to find a process which inhibits the build-up of this coherence, thereby reducing the amount of CARS emission. By using doughnut shaped beams to suppress this build-up, a reduced area of emission (similar to the concept of STED) providing a spatial resolution below the diffraction limit might be obtained.

In the remainder of this chapter, we discuss three schemes, which are modifications of the CARS experiment as described in chapter 4, with the goal of investigating whether these schemes may provide images with sub-diffractionlimited resolution. We perform these theoretical investigations using numerical solutions of density matrix models of these schemes to determine experimentally viable routes to sub-diffraction-limited CARS microscopy. The first scheme investigates local suppression of CARS emission by destructive interference which occurs when an additional light field with the CARS frequency is incident upon the sample simultaneously with the pump, Stokes and probe beam (section 5.2). The second scheme describes the suppression of the build-up of coherence between the ground state and vibrational level by indirectly pre-populating the vibrational level via an incoherent process (section 5.3). The third scheme describes the spatially dependent modulation of the emitted CARS light through periodic ground state depletion, caused by Rabi-oscillations (section 5.4). The concept of each scheme is described in these sections and the enhancement of spatial resolution as obtained by these schemes is discussed.

\subsection{CARS emission suppression by interference}

In a recent report by Nikolaenko et al. [85], it was experimentally shown that CARS emission from a medium can be inhibited by interfering the four-wavemixing process by an additional light field with a carrier frequency equal to $\omega_{\text {cars }}$. They reported that this suppression depends on the intensity of the interfering CARS field and on the phase-lag of this additional CARS field with 
respect to the pump, Stokes and probe fields. They speculate that this suppression might be used to obtain sub-diffraction-limited resolution. Usually, interference processes are measured by, for instance, aligning two separate beams on a detector. The measured intensity, in such a case, depends on the phase difference between the two beams and on the relative intensity. A minimum of intensity is found when two equally intense beams with a mutual phase difference of $\pi$ are incident on the detector. Nikolaenko, however, described an interference effect which occurs in the sample. More specifically, the sample is illuminated by four light beams which induces two interfering processes in the medium. The first process is a CARS process, as induced by a pump, Stokes and probe beam. The second process is a Rayleigh scattering process, as induced by a beam with a frequency equal to the one generated by the CARS process. Destructive interference of the two in the sample means that the induced polarizations generated by the two processes destructively interfere, yielding an induced polarization close to zero and thus also a suppressed emission. Indeed, they experimentally show that the CARS emission can be suppressed by $90 \%$, compared to a standard CARS process, by setting the intensities of the four beams and their mutual phase differences appropriately.

In order to study whether this suppression scheme can be used to obtain images with a sub-diffraction-limited resolution, we use the density matrix approach as described in chapter 4 to model this scheme, and to study the relationship between the parameters of the fields (intensity and phase) and the suppression of CARS emission. As suggested earlier in section 5.1.2, a nonlinear relationship between the applied intensity and the amount of suppression is needed to provide a scheme for sub-diffraction-limited resolution imaging. Although the process of interference of two beams is known to be linear (it is the addition of two fields), the order of the relationship describing the interference process which uses four beams (as in the suggested scheme) is not very obvious.

\subsubsection{Concept and numerical model}

As described by Nikolaenko [85], the experiment consists of a CARS experiment with a pump, Stokes and probe beam with an additional beam superimposed at the CARS emission frequency. In order to find the optimum of destructive interference, they varied the intensity of the additional beam and its phase. To model this experiment, the density matrix model for CARS emission from a sample, as described in chapter 4 , is used in this section in order to find the relation between the suppression efficiency and the phase and amplitude of the light fields.

In our model, the set of the three standard light fields for CARS was extended to four fields. Each light field is specified by a Gaussian pulse shape, a maximum amplitude and a carrier phase. The parameters for the pump, Stokes and probe pulses are kept constant throughout the set of calculations. First, the intensity of the superimposed light field at the CARS frequency $\left(\omega_{\text {cars }}\right)$, which we call the interference beam, is varied keeping the carrier phase con- 
stant, and its effect on the suppression of CARS emission is described in the first part of section 5.2.2. Subsequently, the carrier phase the interference beam is varied, keeping the intensity of the field a constant, and its effect on the suppression of the CARS emission is described in the second part of section 5.2.2. Furthermore, the nonlinearity of this suppression process and its applicability for obtaining a sub-diffraction-limited image are discussed.

\subsubsection{Results}

\section{Intensity dependence of CARS suppression efficiency}

The pulse durations of each of these fields was set to $7 \mathrm{ps}$ and a zero time delay with respect to each other was used (e.g., simultaneously incident on the sample). The maximum intensity of the pump, Stokes and probe beam was kept constant throughout the experiments. The carrier phase of the interference beam was set at $1.5 \pi$ difference with respect to the pump, Stokes and probe phases, since, from additional calculations, it was found that the amount of suppression of the CARS emission reaches a maximum for this value. The intensity of the applied interference beam was varied in order to find the optimal intensity to suppress CARS emission from the sample, and to find the relation between the interference beam intensity and the CARS emission. These calculations were performed using many different parameter settings and a typical example of the results is shown in fig. 5.4 where the CARS emission amplitude, as expressed in terms of $\rho_{\text {cars }}$ (recall section 4.2.2), is given as a function of the amplitude of the interference beam, as expressed in terms of $\chi_{i f}=\frac{E_{i f} \mu_{13}}{\hbar}$.

It was found that the CARS emission is indeed suppressed, by a maximum factor of $75 \%$ with respect to the case where no interference beam is present, for an interference beam amplitude of $\chi_{i f}=0.32 \cdot 10^{12} \mathrm{rad} / \mathrm{s}$, for this particular case, as can be seen in fig. 5.4. The response of the system can be characterized in three regions. In the graph, it is found for the first region, where $\chi_{i f}<$ $0.06 \cdot 10^{12} \mathrm{rad} / \mathrm{s}$, that the CARS emission is hardly effected by the weak applied interference fields. For the second region between $\chi_{i f}>0.1 \cdot 10^{12} \mathrm{rad} / \mathrm{s}$ and $\chi_{i f}<0.5 \cdot 10^{12} \mathrm{rad} / \mathrm{s}$, there is a region of destructive interference. The third region for strong applied fields $\chi_{i f}>0.5 \cdot 10^{12} \mathrm{rad} / \mathrm{s}$, the Rayleigh scattering of the applied $\chi_{i f}$ field becomes the dominant factor in the emission of CARS fields.

In order to determine whether this technique with an interfering beam can be used to obtain images with a sub-diffraction-limited resolution we now compare the relationship between the interference beam intensity and the CARS signal with the two techniques of STED and the reduction of excitation area by higher order nonlinear interaction. Unlike in STED, the relationship does not resemble a saturation process, since for a saturation process the signal intensity is a monotonically decreasing function for increasing applied intensity. Such a saturation does not occur for applied intensities above $\chi_{i f}>0.32 \cdot 10^{12} \mathrm{rad} / \mathrm{s}$, therefore, a spatially distributed saturable suppression process such as by using doughnut shaped beams, similar to that in STED, cannot be obtained 


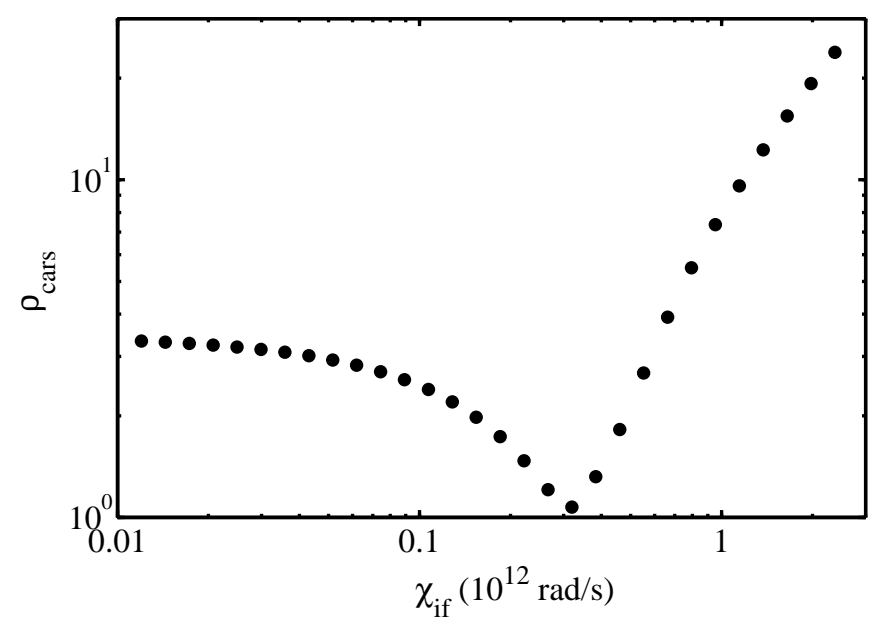

Figure 5.4: Calculated CARS emission amplitude as a function of interference beam amplitude. It can be seen that the CARS emission efficiency is reduced at a certain point where the linear polarization induced by the interference beam destructively interferes with the nonlinear polarization induced by the pump, Stokes and probe beams.

with this interference process and an improved spatial resolution using this route cannot be expected. Furthermore, the increase of the CARS signal for $\chi_{i f}>0.32 \cdot 10^{12} \mathrm{rad} / \mathrm{s}$ follows a polynomial approximately of the order $n=2.5$, using eq. 5.1 a maximal factor of 1.5 for the improvement of the resolution is therefore expected.

The claim by Nikolaenko et al. that the interference process may provide a route for a sub-diffraction-limited resolution actually seems questionable given the fact that the range of transverse $k$-vectors does not increase by the linear superposition of the interference beam, which is a prerequisite to obtain an increased spatial resolution. After elimination of the possibility to use a STEDlike suppression process to obtain an improved resolution we conclude that a significant increase in resolution using an interfering CARS beam is highly unlikely.

\section{Phase dependence of CARS suppression efficiency}

Besides the described intensity dependence, in CARS suppression, also a carrier phase dependence is expected since interference depends on the phase difference between the interfering beams.

To investigate the influence of phase changes, with an interfering beam amplitude of $\chi_{i f}=0.32 \cdot 10^{12} \mathrm{rad} / \mathrm{s}$ (as found to be the minimum in fig. 5.4), 
a set of calculations is performed where the carrier phase of the field is varied between zero and $2 \pi$. As the result of this calculation the CARS emission intensity with respect to this carrier phase difference is shown in fig. 5.5.

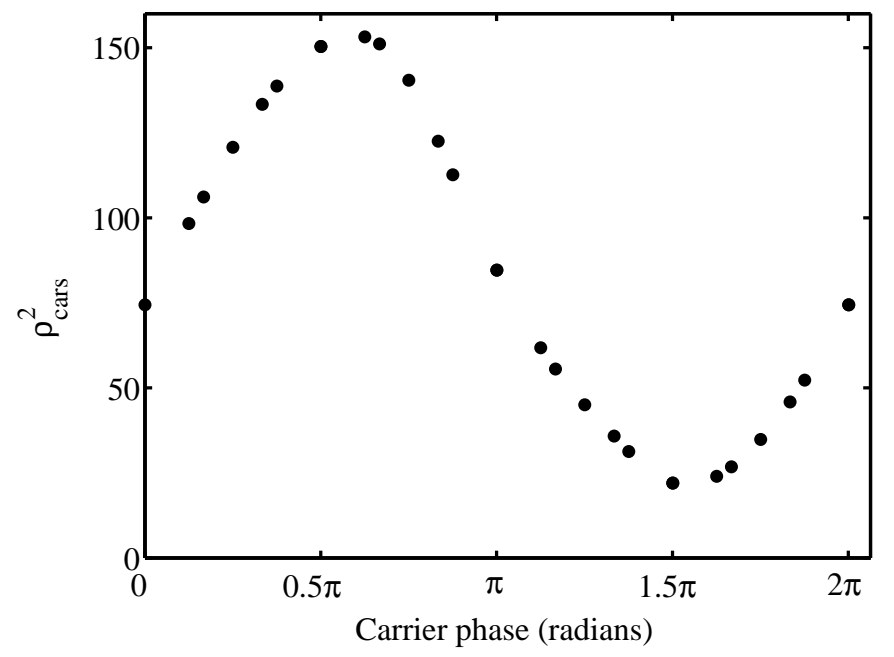

Figure 5.5: Calculated CARS emission intensity as a function of the carrier phase of the interference beam. The CARS emission efficiency is minimized at a carrier phase of $1.5 \pi$ where the scattering of the interference beam destructively interferes with the CARS generation process of the pump, Stokes and probe beam.

It can be seen that the CARS emission suppression varies sinusoidally with the carrier phase difference between the interfering beam and the emitted CARS signal (the latter being fixed by that of the pump, Stokes and probe beams). The minimum of CARS emission efficiency, a reduction of $75 \%$ compared to the case for which no interfering beam is present, is found for a carrier phase difference of $1.5 \pi$. The linear order of suppression induced by this linear superposition of the interference beam is not expected to increase the range of $k$-vectors present in the CARS emission and, therefore, an increase in resolution using such an interference process is not expected.

\subsubsection{Improvement of resolution}

To summarize the previous sections, we here studied the relationship between the intensity and phase of an interfering beam and the influence on the CARS emission due to a pump, Stokes and probe beam, all incident on the sample, to examine a scheme presented by Nikolaenko et al. with regard to its potential for an improved resolution in CARS microscopy. We found that, indeed, as was claimed, CARS emission can be suppressed within a certain window 
of intensities and for specific values of the phase lag between the interfering beam and the pump, Stokes and probe beams. A maximum of destructive interference only occurred for one specific intensity and one specific phase lag. However, the suppression of CARS emission is not saturable since the CARS emission increases again for an increasing interference beam intensity above the value for destructive interference. Therefore, a suppression technique similar to STED cannot be implemented using this interference process. Furthermore, the linear superposition of the interference beam with the CARS emission does not increase the range of $k$-vectors present in the CARS emission which is a prerequisite to obtain an improved resolution.

\subsection{CARS emission suppression by population of vibrational level}

\subsubsection{Concept and numerical model}

The population dynamics which we observe in our initial calculations in section 4.2.4 had confirmed what is named also in the literature as the physical mechanism for CARS emission [88], namely that the two-photon resonance of the pump and Stokes light fields generates vibrational coherence, which radiates CARS emission as a sideband of the probe field. The nature of this process also indicates how to suppress CARS emission: if the system were prepared with equal initial populations in the $|1\rangle$ and $|2\rangle$ states, it is expected that this would suppress the build-up of coherence on that transition, thus preventing CARS emission as well. As a study into this approach, we introduce into the density matrix equations an additional light field, named the control light field, to achieve such an equal population by first populating an additional state, named the control state. The control state, $|4\rangle$, is chosen such that $|2\rangle$ is rapidly populated via non-radiative mechanisms, before the arrival of the pump, Stokes, and probe pulses.

The level scheme of the medium and the driving light fields used in these calculations are shown in fig. 5.6. The four-level system contains a ground state $(|1\rangle)$, a vibrational state $(|2\rangle)$, an excited state $(|3\rangle)$ and the control state $(|4\rangle)$. Transitions between the ground and excited states, the vibrational and excited states as well as the ground and control states are assumed as dipole allowed, while all other transitions are dipole forbidden. We study the following case where the incoherent population exchange rate between levels $|4\rangle$ and $|2\rangle$ is considered to be high $\left(0.1 \cdot 10^{12} \mathrm{rad} / \mathrm{s}\right)$, resulting in an equal distribution of population between those two levels. At a first view this case seems to be special, however, we point out that the vibrational modes of complex organic molecules often cross-relax on a relatively short time scale of nanoseconds and picoseconds [89] and therefore meet the conditions. As a validation of the results obtained with this model, we have included the effect of additional vibrational states (which are typically present in most molecules) and found 
$|3\rangle$

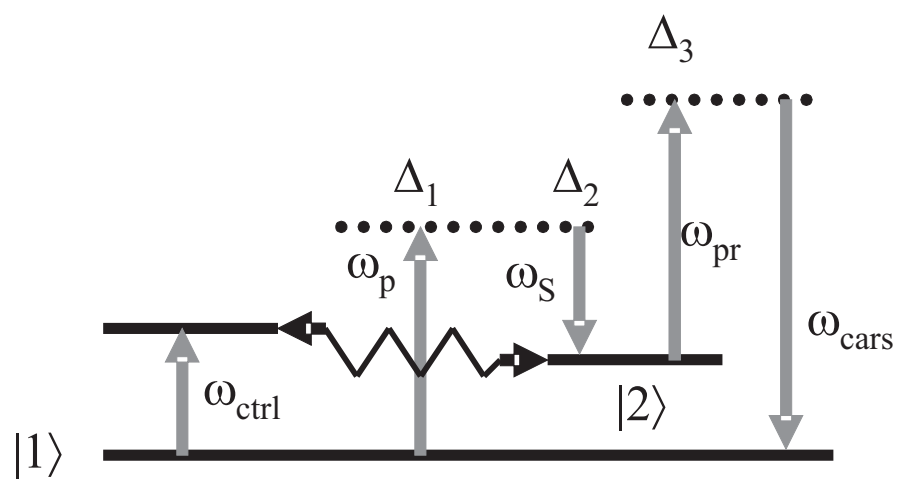

Figure 5.6: Energy level diagram for CARS extended with an additional level $|4\rangle$. Level $|1\rangle$ is the ground level and initially fully occupied, $|2\rangle$ is a vibrational level of the medium, $|3\rangle$ is the excited level and level $|4\rangle$, the control level, which has a fast population exchange with level $|2\rangle$. The vertical arrows between levels indicate possible transitions induced by the corresponding light fields, which are far detuned from the transition $|1\rangle-|3\rangle\left(\omega_{p}, \omega_{S}\right.$ and $\left.\omega_{p r}\right)$, or on resonance with the transition $|1\rangle-|4\rangle\left(\omega_{c}\right)$. Through the nonlinear response of the medium a polarization at the additional $\omega_{\text {cars }}$ frequency is induced, which radiates light (CARS emission) at that frequency. 
that a part of the total population is transferred to these additional states, which reduces the total amount of population involved in the CARS process. However, this only affects the main result insofar that it introduces a secondary suppression of the CARS process.

To understand how the control light field $\left(\omega_{c}\right)$, resonant with the $|1\rangle-|4\rangle$ transition, influences CARS emission, the dynamics of the envelopes of the density matrix elements are calculated with the model analogous to the one described in section 4.2.3. The applied light fields consist of the pump, Stokes and probe fields, and of the control light field, chosen to be resonant with the $|1\rangle-|4\rangle$ transition.

In these calculations it is assumed that there is no temporal delay between the light pulses of the pump, Stokes and probe fields, which all have pulse durations of $2 \mathrm{ps}\left(1 / e^{2}\right)$. In order to prepare the state of the medium, the control pulse chosen to arrive well before the other pulses, in the present example $60 \mathrm{ps}$ earlier, with a duration of 35 ps. The calculation interval extends over 100 ps in steps of 2.5 attoseconds.

\subsubsection{Results}

In this section, we study the relation between the suppression of CARS emission and the intensity of the control pulse, used to populate the vibrational level prior to the arrival of the pump, Stokes and probe beam. The results on the populating effect of the control laser are presented in fig. 5.7 (a), where the population density of the levels $|1\rangle,|4\rangle$ and $|2\rangle$ are plotted versus the pulse area of the control laser pulse. It can be seen that beyond a sufficiently high pulse area the control pulse depopulates the ground state ( $\rho_{11}$ decreasing). At the same time the control pulse populates $|4\rangle$. Importantly it can be seen that, due to the fast redistribution between $|4\rangle$ and $|2\rangle$, also the population density of $|2\rangle$ increases because it closely follows that of $|4\rangle$ with the main result that the populations of $|1\rangle$ and $|2\rangle\left(\rho_{11}\right.$ and $\left.\rho_{22}\right)$ equalize. In the graph it is observed that this process saturates beyond a critical pulse area, which is about 100 radians in the displayed case. As was explained above, such preparation of the medium by the pre-pulse should frustrate the build up of $\rho_{12}$ and should thus suppress CARS emission. Figure 5.7 (b) shows the calculated vibrational coherence $\rho_{12}$ and the corresponding CARS emission intensity when the CARS drive beams are turned on versus the pulse area of the control laser. It can be seen that, indeed, beyond about 100 radians of control pulse area the coherence $\rho_{12}$ is strongly suppressed and, correspondingly, also the CARS emission. The particular example presented in fig. 5.7 shows that CARS emission can be suppressed to a significant extent, here a maximum of $99.8 \%$, compared to the normal intensity of CARS emission.

We found that the saturation intensity, which we define as the intensity of the control beam at which the CARS emission drops to one half of its maximum value [90], corresponds to a $25 \%$ reduction of the ground state population density achieved by the control pulse prior to the CARS process. For comparison 
(a)

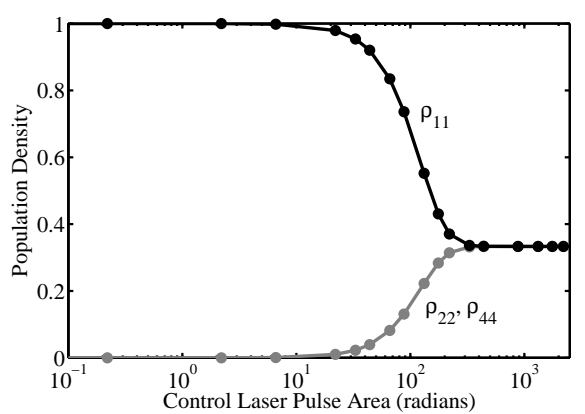

(b)

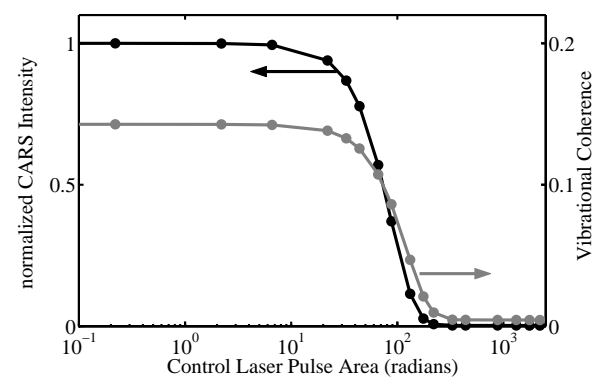

Figure 5.7: (a) Calculated population densities after application of the control pulse. For large pulse areas, a significant fraction of the ground state population is transferred to $|4\rangle$ and, via non-radiative transitions, to $|2\rangle$. (b) Vibrational coherence $\rho_{12}$ (gray) and corresponding intensity of CARS emission (black) after application of the control pulse. The vibrational coherence and CARS emission are suppressed by the control pulse beyond a pulse area of $\approx 100$ radians. 
with required values for the absolute intensities, a ground state depletion of $50 \%$ and population inversion of vibrational states of a liquid biological sample has been demonstrated by Ventalon et al. [91], through direct excitation by a mid-IR laser. Considering the reported pulse duration, pulse energy, and the bandwidths of the vibrational states, we estimate that the populations of their ground state and first vibrational state become equalized at intensities in the order of $70 \mathrm{GW} / \mathrm{cm}^{2}$ during a $100 \mathrm{fs}$ pulse. However, it should be noted that for pre-populating the vibrational state, the pulse area of the control light field is more important than the peak intensity, thus, in our calculations, where a $35 \mathrm{ps}$ pulse duration is considered, the intensity is just $200 \mathrm{MW} / \mathrm{cm}^{2}$. This is well below the threshold for multiphoton ionization and excitation in the mid-infrared. A considerable advantage of this excitation scheme is that the molecule remains in its electronic ground state which decreases the risk of photodamage to occur.

\subsubsection{Improvement of resolution}

The improvement of spatial resolution that would be gained with the described CARS suppression can be estimated by considering an experimental setup wherein the control light beam illuminates the sample with a donut mode, focused to a diffraction-limited spot. The CARS emission from the node with an area defined by where the control light field intensity is lower than the saturation intensity is not suppressed. Emission from outside the node is strongly suppressed for which we assume here a suppression of $99.8 \%$ as in the example in fig. 5.7. The resolution can then be considered to be defined by the radius of the node $\left(r_{i n}\right)$ when the contribution to the measured CARS intensity of the emission from within the node is greater than the contribution of emission from outside $\left(r_{\text {out }}\right)$ the node (for this case $r_{\text {in }}=\sqrt{\frac{100 \%-99.8 \%}{100 \%}} r_{\text {out }}$ ). From our numerical results, we derive an upper limit to the improved resolution of approximately $\frac{\lambda}{(22 \cdot \mathrm{NA})}$ where NA is the numerical aperture of the imaging system and $\lambda$ is the wavelength of the probe beam (since the wavelength of the probe beam is the dominant factor that sets the diffraction limit for CARS imaging). Furthermore, it was observed that the intensity of all other non-linear emission processes (recall fig. 4.2) are similarly suppressed which seems to offer the possibility that spatial resolution of CSRS microscopy may be increased as well.

Regarding CARS microscopy, we conclude from our calculations that subdiffraction-limited resolution images can be obtained with a suitably shaped control light beam with a node at its center (a doughnut shaped beam (see section 5.1.2)), resonant with the $|1\rangle-|4\rangle$ transition and with an intensity above the saturation level of the vibrational transition of interest. Then, the node at the center leaves the CARS process unaffected in a sub-diffractionlimited space around the center of the common focus, whereas CARS emission from that area outside the node is suppressed. The measured CARS emission is then attributed to this sub-diffraction-limited region, where the intensity is 
less than the saturation intensity.

\subsection{CARS emission modulation by Rabi oscil- lations}

As shown in the previous sections, the route to obtain images with a subdiffraction-limited resolution consists of finding a nonlinear relationship between the CARS emission by the medium and the intensity of an applied light field. In this section another optical response (besides saturation), which scales with the intensity of the beam is considered as a route to obtain high resolution images. In the previously described cases, the additional control light field acted upon a transition in the overdamped regime, e.g., the decoherence rate of the transition or decay rates exceeded the stimulated absorption and emission rates of the light field (recall eq. 5.2-5.6). In the following section, we investigate the opposite underdamped case where the damping is small and a Rabi oscillation takes place. The intensity dependence of the Rabi oscillation frequency of the system will be used to spatially label CARS emission. As will be shown through a numerical model, the CARS emission as induced by a pump, Stokes and probe beam obtains sidebands in the frequency regime due to the presence of an additional control beam. The splitting of the sidebands is shown to depend on the location of the emitter with respect to the center of the control beam. Furthermore, the effectiveness of this technique to obtain a sub-diffraction-limited resolution image using this modulation technique is discussed.

\subsubsection{Concept and numerical model}

The dynamical response of the population transfer in the underdamped situation (the resonant Rabi frequency $\chi$ exceeds the transition decoherence rate) show an oscillatory behavior named Rabi oscillations [44]. We propose that this Rabi oscillation can be used to modify a CARS experiment in order to obtain sub-diffraction-limited resolution as follows. A control beam on resonance with a transition origination from the ground state of the molecule induces Rabi oscillations which periodically deplete the ground state population. Simultaneously, a CARS process is induced using a pump, Stokes and probe beam. It is expected that, since the CARS process is based on a vibrational coherence that requires some ground state population amplitude, the periodical depletion of the ground state will periodically alter the CARS emission. As a result, the spectrum of the emitted light from the molecule is expected to show a CARS emission line accompanied by two sidebands with a splitting equal to the frequency of the Rabi oscillation. By using a control beam with a Gaussian shaped spatial intensity profile, the location of the molecule with respect to the center of the control beam can be calculated from the amount of splitting of the sidebands, since the induced Rabi oscillation is mapped spatially by the 
intensity profile of the control light field.

For a theoretical investigation of this dynamics we use a level scheme similar to the one in section 5.3. In summary, the four-level system (see fig. 5.8) contains a ground state $|1\rangle$, a vibrational state $|2\rangle$, an excited state $|3\rangle$ and a control state $|4\rangle$. The applied light fields consist of a pump, Stokes and probe field in combination with an additional control light field that couples the control level, $|4\rangle$, to the ground-state level $|1\rangle$. We use a numerical model similar to the one described in section 4 using the parameters as specified in section 4.2.3. Additionally, for this particular case, an angular frequency $\omega_{14}$ of $565 \cdot 10^{12} \mathrm{rad} / \mathrm{s}$ (approx. $3.33 \mu \mathrm{m}$ or $3,000 \mathrm{~cm}^{-1}$ ) is chosen for the $|1\rangle-|4\rangle$ transition. Although $|4\rangle$ is considered to be a vibrational state in this calculation, such as in [91] or [92], any dipole allowed state can be used. The lifetime of state $|4\rangle$ such as given by the spontaneous emission from state $|4\rangle$ or collisional redistribution to other states is taken to be on the order of nanoseconds [76]. All other parameters are equal to those described in section 4.2.3 except for the cross relaxation rate between states $|4\rangle$ and $|2\rangle$ which is set to ns as well. For these calculations all the light pulse durations $\tau$ are set to $7 \mathrm{ps}\left(1 / e^{2}\right)$ except for the control light field, which we have chosen to be a continuous wave for convenience, although the same results are obtained also for a pulsed control laser when its pulse duration is long compared to that of the other pulses. The simulations extend over $30 \mathrm{ps}$ in steps of $0.1 \mathrm{fs}$.

\subsubsection{Results}

We performed a series of calculations of the emission spectrum around the CARS frequency. In these calculations $\omega_{c}$ was taken to be resonant with the $|1\rangle-|4\rangle$ transition (the detuning $\Delta_{14}=\omega_{14}-\omega_{c}=0$ ), while the decoherence rate of this transition was set to $\Gamma_{14}=0.1 \cdot 10^{12} s^{-1}$ [92]. The amplitude of the control field and, hence, the induced Rabi frequency $\left(\Omega_{R}\right)$ were varied in steps. The results are shown in fig. 5.9 (a) where the spectrum around the CARS frequency, as emitted by the medium, for a number of values of $\Omega_{R}$ is given. A single emission peak is seen at low Rabi frequencies and indicates standard CARS emission, as expected. It can be seen that, for increasing $\Omega_{R}$, the spectrum shows two symmetrically placed sidebands. From our calculations, we find that these sidebands show a spacing from the CARS carrier frequency that coincides with $\Omega_{R}$, as given by eq. 2.25 .

For observing such Rabi splitting of CARS it is critical that the Rabi oscillation frequency exceeds the decoherence rate of the control transition. This can be seen in fig. 5.9 (b), where the spectrum of the CARS emission for increasing values of the decoherence rate is shown. However, it can also be seen that, even in the presence of sub-picosecond decoherence times, the fixed $4 \cdot 10^{12} \mathrm{rad} / \mathrm{s}$ modulation we have assumed is still detectable. This is important, because the decoherence times in a liquid environment such as typically present in biological samples, are very short, with recent observations suggesting that also longer dephasing times can be found, such as on the order of 5 ps [92]. Generally, a 


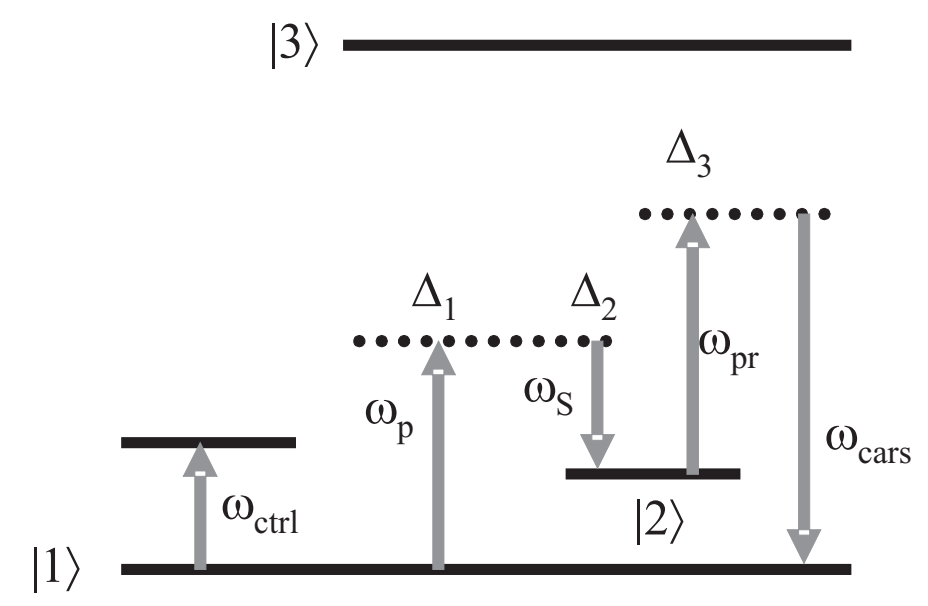

Figure 5.8: Energy level diagram for a CARS process with an additional level $|4\rangle$. Level $|1\rangle$ is the ground level and initially fully occupied. Level $|2\rangle$ is a vibrational level of the medium, $|3\rangle$ is the excited level and level $|4\rangle$ the control state. The vertical arrows between levels indicate possible transitions induced by the appropriate light fields, which are shown far detuned from the transition frequency $\left(\omega_{p}\right.$, $\omega_{S}$ and $\left.\omega_{p r}\right)$, or on resonance with the transition $\omega_{c}$. Through the nonlinear process the medium obtains a polarization at the additional $\omega_{\text {cars }}$ frequency, which is radiated by the medium. The additional control laser at frequency $\omega_{c}$ is used to induce Rabisidebands in the CARS emission spectrum to serve as an optical labeling for an improved spatial resolution. 
large Rabi frequency has the benefit that the frequency splitting between the carrier CARS frequency and its sidebands are easy to distinguish.

\subsubsection{Improvement of resolution}

The induced sidebands can be used to obtain positional information by noting from eq. 2.25 that the Rabi splitting of the sidebands depends on the intensity of the control light field. Furthermore, the geometrical alignment of the molecules under inspection and thus the molecular dipole moment $\mu_{14}$ may be obtained by rotation of the polarization angle of the control light beam, providing additional information on the structure of the sample. Considering a single emitter illuminated by a Gaussian beam, we note that the radial distance of the emitter from the center of the control beam can be obtained by measuring the frequency offset of the sidebands versus the CARS carrier frequency, which can be called a Rabi-labeling of the emitter position. Such a spatially-dependent Rabi frequency is a well-known effect [93], however, it is usually considered to cause undesired broadening and is eliminated, e.g., in pump-probe experiments, by using a much larger pump beam as compared to the probe beam [94].

The absolute position of an emitter location can be calculated by trilateration. We illustrate this with a two-dimensional example, however, a similar approach also applies to three dimensions. Consider an emitter located at the Cartesian coordinates $x_{1}$ and $y_{1}$. The observed CARS emission spectrum, with its sideband frequency depending on the local intensity of the control light beam, will not determine these coordinates but instead provides the distance from the control beam center, $r_{1}=\sqrt{x_{1}^{2}+y_{1}^{2}}$, which is a ring of possible locations centered around the control beam. By scanning the control light beam a known distance $d x$ along the $x$ axis, the radial location of the emitter is changed to $x_{1}-d x, y_{1}$ where it experiences a different control intensity and a different Rabi frequency. As a result, the observed sideband frequency will change, revealing a new distance, $r_{2}=\sqrt{\left(x_{1}-d x\right)^{2}+y_{1}^{2}}$. The expressions for $r_{1}$ and $r_{2}$ can then be solved for $x_{1}$, and a subsequent scan along $y$ by a known $d y$ determines $y_{1}$ as well. Turning our attention to the spatial resolution of such a positioning scheme, we show that the scheme can provide CARS with sub-diffraction-limited resolution.

We note that the control light beam, resonant with the $|1\rangle-|4\rangle$ transition and focused to a diffraction-limited Gaussian-shaped intensity profile, centered on the other light beams, illuminating a distribution of emitters, will generate a distribution of Rabi sidebands on the CARS frequency. If the transverse spatial distribution of the control light field has on its beam axis a peak envelope field strength of $E_{0}$ and a $1 / e$-spot-size of $w_{0}$, then the range of observed Rabi frequencies within the diffraction-limited spot is $\Omega_{\max }=\left|\mu_{14} E_{0} / \hbar\right|$ in the center to $\Omega_{\min }=1 / e\left|\mu_{14} E_{0} / \hbar\right|$ at radial distance $w_{0}$. The radial resolution is then determined by how accurately the Rabi splitting frequency can be measured in the CARS spectra. Given a Rabi frequency, $\Omega$, corresponding to a radial distance from the center of the focus of $r$, and a frequency measurement 


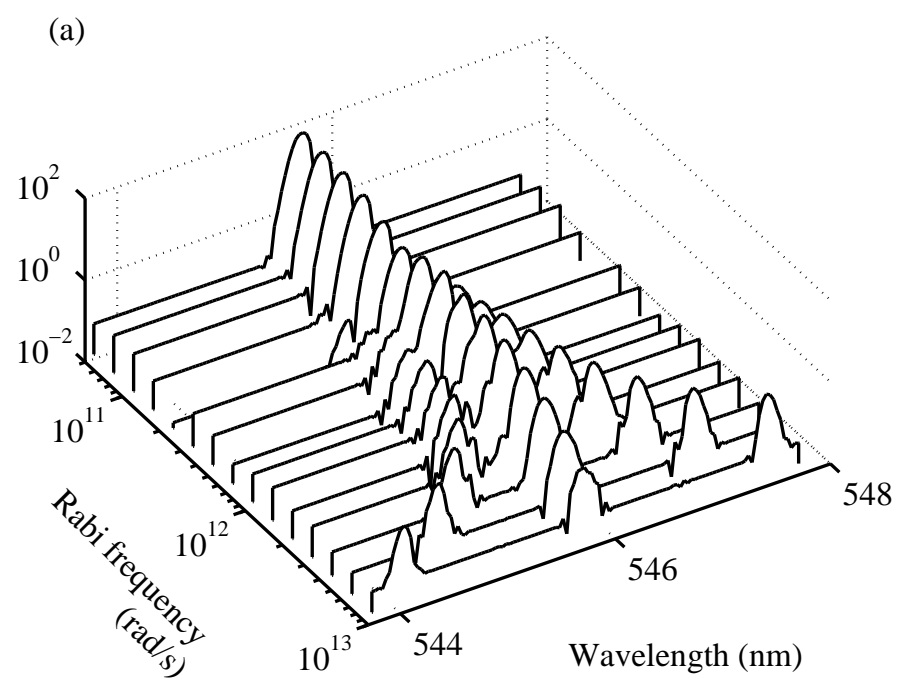

(b)

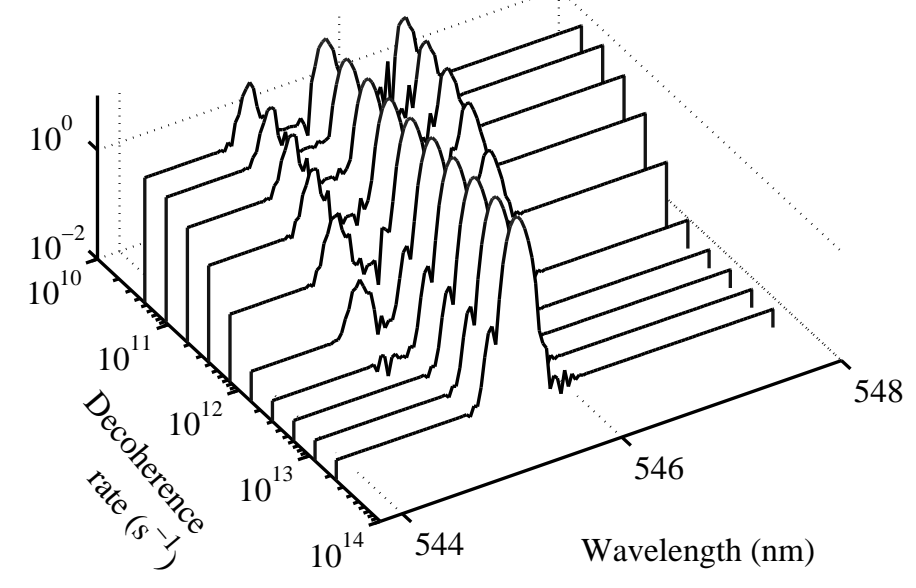

Figure 5.9: (a) Calculated CARS emission spectrum for $\Gamma_{14}=0.1 \cdot 10^{12} s^{-1}$. The splitting of the sidebands is proportional to $\Omega_{R}$. (b) Calculated a CARS spectra with a fixed $\Omega_{R}=4 \cdot 10^{12} \mathrm{rad} / \mathrm{s}$ and various values of the decoherence rate of $|4\rangle$. Note that the amplitude of the sidebands decreases until they entirely disappear around where $\Omega_{R}$ equals the decoherence rate. 
accuracy of $d \Omega$, we derive the corresponding radial resolution of $d r$ as follows. The radial distribution of the electric field of a Gaussian control beam is given by

$$
E(r)=E_{0} e^{-\frac{r^{2}}{w_{0}^{2}}}
$$

At two points within this field located at $r_{1}$ and $r_{2}=r_{1}+d r$, the field induces Rabi oscillations with frequencies as given by

$$
\begin{aligned}
& \Omega_{1}=A E_{0} e^{-\frac{r_{1}^{2}}{w_{0}^{2}}} \\
& \Omega_{2}=A E_{0} e^{-\frac{r_{2}^{2}}{w_{0}^{2}}}
\end{aligned}
$$

where $A=\frac{\mu}{\hbar}$. Solving both of these for the argument of the exponential gives

$$
\begin{aligned}
-\frac{r_{1}^{2}}{w_{0}^{2}} & =\ln \left(\frac{\Omega_{1}}{A E_{0}}\right) \\
-\frac{r_{2}^{2}}{w_{0}^{2}} & =\ln \left(\frac{\Omega_{2}}{A E_{0}}\right)
\end{aligned}
$$

Using the laws for division and multiplication of logarithmic terms gives

$$
\begin{aligned}
-\frac{r_{1}^{2}}{w_{0}^{2}} & =\ln \Omega_{1}-\ln A E_{0} \\
-\frac{r_{2}^{2}}{w_{0}^{2}} & =\ln \Omega_{2}-\ln A E_{0}
\end{aligned}
$$

Adding equations 5.13 and 5.14 gives

$$
\frac{r_{2}^{2}-r_{1}^{2}}{w_{0}^{2}}=\ln \frac{\Omega_{1}}{\Omega_{2}}
$$

Now, we set $\Omega_{2}=\Omega_{1}-d \Omega$, and substitute for $r_{2}$ and $\Omega_{2}$ and expand the polynomial, which then becomes

$$
\frac{r_{1}^{2}+2 r_{1} d r+d r^{2}-r_{1}^{2}}{w_{0}^{2}}=\ln \frac{\Omega_{1}}{\Omega_{1}-d \Omega}
$$

Solving for $d r$ gives

$$
d r=\frac{w_{0}^{2}}{2 r+d r} \ln \left(\frac{\Omega}{\Omega-d \Omega}\right)
$$

The transcendental equation 5.17 shows that $d r$ becomes smaller than the diffraction-limit (smaller than $w_{0}$ ), and that higher Rabi frequencies lead to higher resolution. Furthermore, emitters located away from the center of the control beam are resolved better than emitters close to the center (where $r \rightarrow 0$ 


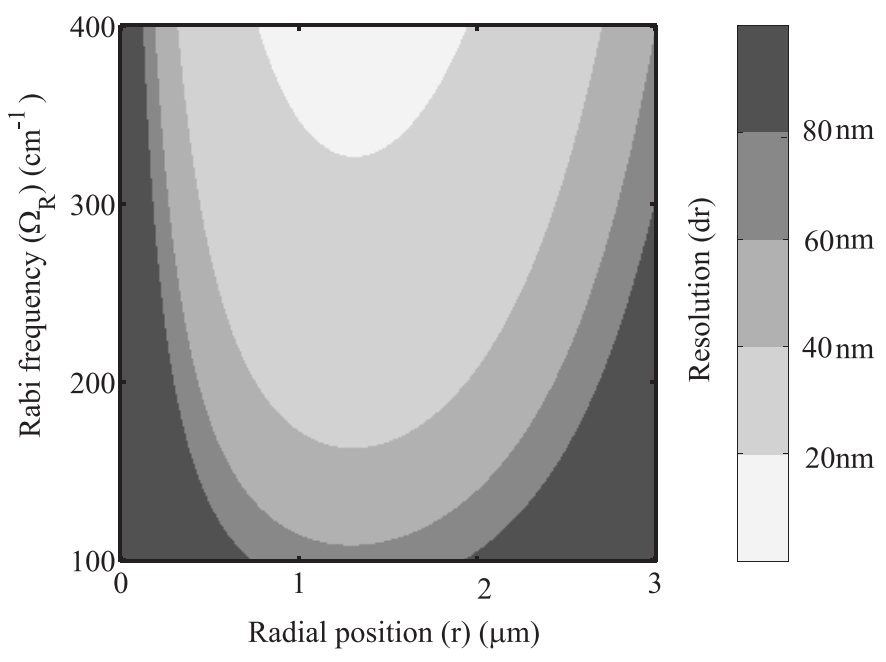

Figure 5.10: Spatial resolution as a function of the Rabi frequency and radial location of the emitter.

and $d r$ becomes large). The resolution is expected to be maximal at the steepest intensity slope of the control beam, therefore, by using a Gaussian shaped control beam, the highest resolution is expected to be found off center. This is illustrated in Fig. 5.10 where we have plotted the Rabi-labeling resolution, $d r$, as a function of both Rabi frequency and radial location, for $d \Omega=3 \mathrm{~cm}^{-1}$ (which is a typical value for the linewidth of the CARS peak in a spectrum). This figure shows that considerable improvement over diffraction-limited CARS microscopy is possible.

To make the improvement explicit, we consider a specific example for a microscope with a numerical aperture of 1.2 and wavelengths that were used in our calculations. In this case, the diffraction-limited resolution is $171 \mathrm{~nm}$. If the control light beam $(\lambda=3.3 \mu \mathrm{m}$, focused to a diffraction-limited spot, induces $100 \mathrm{~cm}^{-1}$ Rabi oscillations, then at a radial distance of $1.2 \mu \mathrm{m}$, the resolution is $65 \mathrm{~nm}$. We estimate from the data presented in [92] that the light field intensity required to generate a Rabi frequency of $100 \mathrm{~cm}^{-1}$ is about $500 \mathrm{MW} / \mathrm{cm}^{2}$, which is comparable to the intensities used in standard CARS experiments, and sufficiently low to avoid multiphoton excitation [91]. However, it should be noted that such a value will depend on the molecular system under study.

\subsection{Conclusion}

In this chapter, several routes to integrate CARS microscopy with sub-diffraction-limited resolution techniques have been evaluated by solving appropriate 
density matrix models.

We have studied the interference process, as described by Nikolaenko et al. [85], and verified their claim that this scheme suppresses CARS emission from a medium. We found, however, that this type of interferometric suppression is not suitable for sub-diffraction-limited resolution imaging, since the relation between the amount of suppression and applied intensity is of linear order. The suppression depends critically on the intensity and phase of the interference beam, making it difficult to determine a practical experimental configuration that could suppress CARS emission in a spatially selective manner.

We have theoretically demonstrated a route to obtain sub-diffraction-limited resolution in CARS microscopy that is analogous to STED. We have shown that an intense control laser beam can be used to suppress CARS emission, when the control laser pre-populates an additional vibrational state that is nonradiatively coupled to the vibrational state and probed by the CARS process. For neighboring vibrational states that are coupled with high transitional probabilities, as can routinely be found for aqueous biological samples, a saturation process analogous to that of STED is observed. Our calculations, based on typical parameters for molecular transitions used in CARS microscopy, showed that high saturation levels of up to $99.8 \%$ might be obtained, which corresponds to an improved resolution of $\frac{\lambda}{22 \cdot \mathrm{NA}}$. The required intensities for the control beam, which depend on the pulse area, span a range from $200 \mathrm{MW} / \mathrm{cm}^{2}$ through to $100 \mathrm{GW} / \mathrm{cm}^{2}$, depending on the pulse duration, which seems tolerable because the molecules remain in their electronic ground state which limits the risk of photodamage to occur.

As an alternative approach, not based on saturable suppression, but based on a spatially dependent amplitude modulation due to induced Rabi oscillations, we have demonstrated a second route to obtaining sub-diffraction-limited CARS images. By resonantly driving the excitation of a control state with relatively long decoherence times, intensity and, thus, spatially dependent sidebands in the CARS emission spectrum can be generated. By accurately measuring this Rabi splitting in the CARS spectrum, objects can be resolved to a resolution below the diffraction-limit. Using typical numbers, we have shown that a resolution in the order of $65 \mathrm{~nm}$ may be achievable. We note that our calculations also show the generation of Rabi sidebands in other molecule-specific emission lines, such as Coherent Stokes Raman Scattering. The approach towards sub-diffraction-limited resolution presented here can thus be used with that microscopy technique as well, making it more broadly applicable. 
$\bigoplus$ 


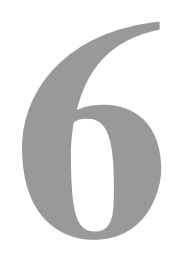

\section{Numerical model of SIT solitons in $\Lambda$-type media}

\subsection{Introduction}

Strong and coherent interactions between $\Lambda$-type three-level atomic media and resonant light pulses have been a topic of much interest recently [26-28]. This interest has been driven by quantum memory systems [29, 30], quantum logic operations [31], and image storage [32]. In the case of quantum information processing, where it is necessary to store and process multiple quantum bits (qubits) in the same medium $[33,34]$, it is of great interest to examine the influence of multiple light pulses on the spatial distribution of the ground-state coherence of the medium.

In view of the latter, the use of solitons based on self induced transparency (SIT solitons) [35] appears to be an interesting candidate for continuous variable quantum computing [36]. Continuous variable optical computing (quantum or classical) requires a strong nonlinear optical interaction [36]. Due to its general applicability the non-resonant Kerr effect with its inherently low interaction strength is chosen as the nonlinear optical interaction [37,38], however this requires that a combination of rather intense optical pulses and long interaction lengths are used [39,40]. SIT is a resonant effect [35], thereby providing a much stronger interaction. This offers the possibility of reducing the interaction lengths and pulse intensities required for continuous variable optical computing.

A further disadvantage of using the non-resonant Kerr effect is that any information is stored in optical pulses that propagate with the speed of light. Operations between different pulses must be carefully timed so that the overlap 
between them in the linear (e.g., beam-splitters) or nonlinear optical elements (the Kerr medium) is maximized [40].

However, as will be shown here, in the case of SIT solitons, the timing problem is much less critical because SIT solitons can be captured, set to a full stop and thereby become stored in the (non-propagating, stationary) ground state coherence of a three-level medium by means of an appropriate applied control light field $[41,42]$. New results, that will be presented in chapter 7, show that sets of such SIT solitons allow for computational operations to be performed on the stationary coherence, rather than on the traveling soliton. In addition, we show that multiple trapped SIT solitons, when released, propagate as a coupled set.

In the present chapter we present the theoretical model on which we base our studies of soliton propagation, soliton capture and storage and the named computational operations. The model uses the one-dimensional density and Maxwell equations to describe the propagation and interaction of pulses in a $\Lambda$-type medium (see fig. 6.1). In the following, the model is described and the motivation for the choices for various parameters and approximations is given. Next, the model is validated by showing that it reproduces well-known features of SIT solitons in effective two-level and three-level systems. After these tests we start to present some basic ingredients for our soliton computing scheme. The first is to show how an SIT soliton can be captured in a three level system. Furthermore, such capture events result in a relationship between the phase of the soliton before its capture and the phase of the ground state coherence which is written into the medium upon such capture events.

In the next chapter (ch. 7), new results on the dynamical behavior of soliton propagation with multiple SIT solitons are presented. It will be shown that the interaction of a set of SIT solitons can be used as a component in all-optical computing.

\subsection{Numerical model}

There are various models to describe the nonresonant propagation of light pulses through a nonlinear optical medium that can be implemented in a numerical model, such as the so-called coupled wave equations [43] where the nonlinear effect of the medium on propagating light fields is approximated as material dependent coefficients. These models do not describe that the involved (incident or generated) light fields may significantly change the state of the medium itself, which is to be expected particularly when considering the strongest interactions possible, achieved by feeding all of the involved light frequencies on resonance with transitions in the medium. An example of this is that the population of the medium's energy level might change, and these population changes become maximum when the involved light fields are intense and resonantly excite optical transitions. Since these changes to the medium may influence the propagation characteristics we choose here a model that includes such changes to the medium. In particular, to include such strong effects 


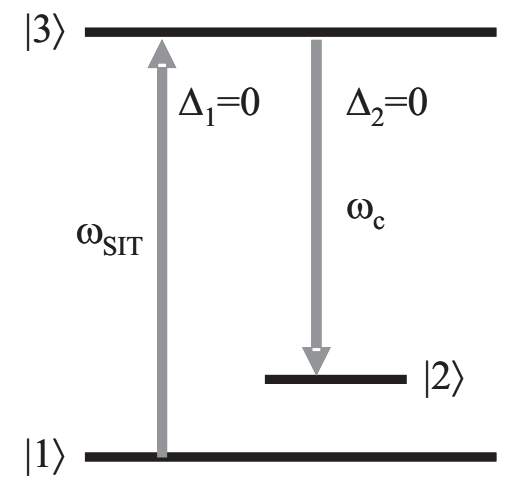

Figure 6.1: The $\Lambda$-type system consists of two ground states $|1\rangle$ and $|2\rangle$ and an excited state $|3\rangle$. Transitions of population density between these levels is stimulated by the application of light fields resonant with the transitions $\left(\omega_{S I T}\right.$ for the initially occupied $|1\rangle-|3\rangle$ transition and $\omega_{c}$ for the initially empty $|2\rangle-|3\rangle$ transition).

in the three-level medium described below, where the light fields are resonant with the transitions of the medium, we describe the medium via its density matrix.

The Maxwell equations (wave equation), which describe the propagation of light, use the polarization of the atoms in the medium as a source term. Changes of this polarization due to a light field are usually described by use of the density matrix equations, which are derived from the Schrödinger equation [44]. Essentially, to describe the pulse propagation dynamics consistent with the polarization and population state of the three-level medium, it is required to simultaneously solve the coupled set of differential equations consisting of the electro-magnetic wave-equation and the density matrix equations.

In these calculations, we consider that the medium's constituent atoms are considered to remain at rest which is a good approximation, e.g., for solidstate media [95] or ultracold gases [26]. The medium is considered to have two ground states $(|1\rangle,|2\rangle)$ and one excited state $(|3\rangle)$. We use the usual description of a $\Lambda$-type medium, where the $|1\rangle-|3\rangle$ and $|2\rangle-|3\rangle$ transitions are dipole allowed and radiative, while the $|1\rangle-|2\rangle$ transition is dipole forbidden. Such a medium is described by the standard set of density matrix equations as described in section 2.2.4. The solution of these equations provides the time dependent population densities and coherences between populations throughout the medium.

The medium is assumed to be driven by two different light fields $E_{S}$ (the SIT field) and $E_{c}$ (the control field) with carrier frequencies $\omega_{S I T}$ and $\omega_{c}$ respectively that travel as plane waves in the $+z$-direction.

The calculations typically use a value of the product of $N \mu=1.7 \cdot 10^{-10} \mathrm{Cm}^{-2}$ 
[96], which would be obtained, for instance, by probing the transition from state $4^{1} \mathrm{P}$ to state $3^{1} \mathrm{D}$ of calcium in a gas-cell with a partial pressure of $30 \mathrm{mBar}$ of calcium. The equations were then integrated over a propagation length of $500 \mu \mathrm{m}$, over which the medium is almost perfectly opaque for weak light on the $|1\rangle-|3\rangle$ transition.

The calculations are performed numerically using a fourth-order RungeKutta algorithm implemented in the programming language of $\mathrm{C}++$ and are performed over a grid of points in space and time. At each grid location along the propagation axis, the medium's state is represented by an independent density matrix. All calculations begin with the ground state $|1\rangle$ fully occupied $\left(\rho_{11}=1\right)$ throughout the entire medium (for all $\left.z\right)$ and no coherence between any of the levels $\left(\rho_{12}=\rho_{13}=\rho_{23}=0\right)$.

\subsection{Test of the numerical model}

In the following, we describe how the model was validated by comparing its results with those reported in the literature, as will be detailed below. For example, it is known since long [35] that when a particular pulse shape is chosen and a particular pulse area, defined as the integral of the electric field over time, this pulse can propagate through a strongly absorbing (opaque) medium and experiences very little loss although the light is resonant with the optical transition. A simplified description of this phenomenon is that the first half of the pulse becomes continuously absorbed during propagation but that the absorbed energy is fully returned to the pulse at its trailing edge, thereby only reducing the group velocity of the pulse. This surprising phenomenon has been termed self-induced transparency, SIT.

It has been shown that the shape of the stable solution of such an SIT soliton is a secant hyperbolic function [35]. The shape invariance and also the invariance of the pulse area when two such pulses have interacted with each other is often termed particle-like properties, thus the name soliton. A closer inspection of SIT solitons has shown that a soliton breaks up into several solitons whenever the pulse area exceeds $3 \pi[35,97]$. Similarly, if the pulse area is between $\pi$ and $3 \pi$, the pulse reshapes to a longer or shorter duration by a corresponding change of the peak electric field (and peak power), and then travels with constant sech-shape and a pulse area of $2 \pi$. Note that SIT solitons with $2 \pi$ pulse area can still occur with different peak powers and pulse durations. It was found that the propagation velocity of a soliton is typically much slower than the velocity in vacuum. Also, the soliton velocity decreases with decreasing peak power (and thus increasing pulse duration). A fast soliton, overtaking another, slower soliton within the medium, does not alter the amplitude and propagation speed of either soliton [97]. In the following, we show that our model replicates each of these characteristic properties of SIT solitons. 


\subsubsection{Soliton propagation}

A temporal optical soliton on resonance with a transition of a two-level medium propagates without loss of energy [35]. This process was first described by McCall and Hahn and can qualitatively be understood as follows [35]. Initially the light of the leading edge of the pulse is absorbed by the medium, by exciting the entire population density from the ground state towards the upper state. The trailing edge of the pulse finds the medium in the excited state (population inversion) and is amplified by the medium through the process of stimulated emission, resulting in a complete de-excitation of the population density back to the ground state, thus a complete Rabi cycle. After completing this cycle the medium has not gained energy since the absorption and re-emission of light is exactly balanced, therefore, the light pulse propagates without energy loss through the medium. However, a certain amount of time is needed to complete one full cycle, which can strongly reduce the pulse propagation velocity of such an optical soliton as compared to the propagation speed of light in a weakly absorbing medium or vacuum [35,97]. The assumption that the soliton propagates without loss is valid for the condition that $\tau_{21} \ln (2) \gg t_{m}$ where $\tau_{21}$ is the lifetime of the excited state and $t_{m}$ is the time the light remains in the medium. As an example of the lifetime of a medium where such solitons can be formed is the $5 \mathrm{P}_{3 / 2}$ state of ${ }^{87} \mathrm{Rb}$ with a lifetime of $27 \mathrm{~ns}$ [98]. The examples of calculations presented in this chapter have been performed assuming such long lifetimes. From these calculations we observe soliton behavior with pulse propagation times of up to about 1 ns through the medium, to be short enough for the approximation of lossless transport of the soliton.

As a test of the numerical model, the propagation of a soliton in a medium was modeled over a wide range of parameters. In fig. 6.2 we depict a typical example. In this case, at $\tau_{0}=0.2 \mathrm{~ns}$ a pulse with a duration of $200 \mathrm{ps}$ and a hyperbolic secant shape is launched into the medium at the front end and the resulting evolution of the electric field distribution in space and time is plotted as gray-scale (white means highest field and black means zero field). This figure shows that the pulse propagates without noticeable loss through the medium until it exits the medium at the rear end. From the relatively steep slope of the white line compared to the dashed line indicating the speed of light in vacuum, it can be seen that the propagation velocity of the soliton is much smaller than $c$, the speed of light in vacuum. In the displayed case the soliton velocity is approximately $c / 330$.

\subsubsection{Secant hyperbolic pulse shape}

By solving the equations of the dynamics of a two-level system analytically and when neglecting residual absorption, McCall and Hahn have proven that the stable solution of the soliton pulse shape is a hyperbolic secant function [35]. This shape is the result of the cyclic motion of the population of the medium from the ground state towards the excited state and back due to the presence of the light field. Mathematically seen, the population difference is driven by 


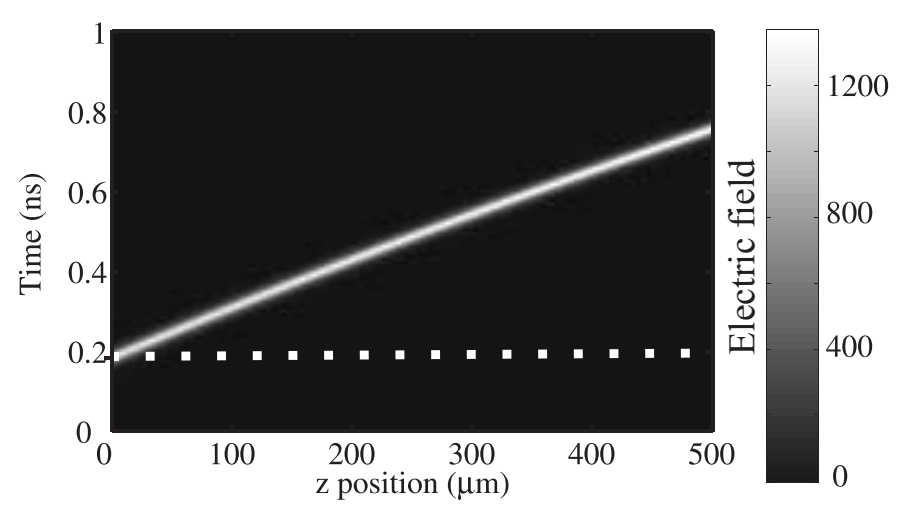

Figure 6.2: Calculated propagation of an SIT soliton through a three-level medium. The sech-shaped pulse, launched into the medium at $\tau_{0}=0.2 \mathrm{~ns}$ on the left side in the figure, propagates undisturbed with an almost constant velocity through the medium and exits it on the right side. The dotted line indicates propagation at the speed of light in vacuum, $c$.

the product of the induced atomic polarization and the light field amplitude. Similarly, the polarization is driven by the product of the light field and the population difference. Due to this mutual influencing, the coupled system of the light field, polarization and population difference has a natural oscillatory behavior which is called Rabi oscillation and was discussed also in chapter 2. Here, however, if the light is pulsed, with a sech-shape and pulse area of $2 \pi$, only one Rabi-cycle is performed at each location in the medium when the soliton passes.

Another important finding of McCall and Hahn is that a pulse that initially does not have a hyperbolic secant shape, still evolves into this shape of an SIT soliton after some propagation distance. This is explained as follows. A pulse that deviates from the hyperbolic secant shape leaves the medium, after the passage of the pulse, partially excited. Through this process, the pulse looses energy to the medium during its propagation until the pulse has reached the stable form of the hyperbolic secant shape.

As a test for our model we calculated the propagation of non-sech-shaped pulses and observed the development of the temporal shape, such as is shown in fig. 6.3. It can be seen that starting the propagation dynamics with a Gaussian shaped pulse reveals a re-shaping into a pulse of almost sech-shape. Similar tests, done with other initial pulse shapes showed that always a reshaping takes place into the hyperbolic secant shape of an SIT soliton. 

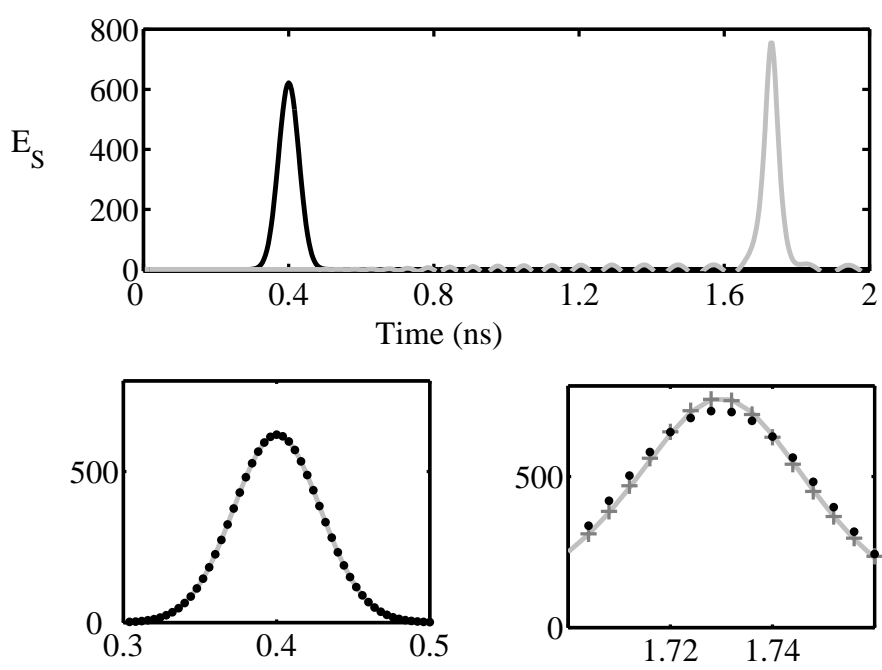

Figure 6.3: (top) Gaussian shaped temporal profile of the electric field as launched into the medium at $\tau_{0}=0.4 \mathrm{~ns}$ (black) and temporal profile after propagation of $400 \mu \mathrm{m}$ (gray) into the medium. Thereafter, the pulse propagates as an SIT soliton with near sechshape through the medium. The temporal shape of the inserted pulse (gray line in lower left panel) matches a Gaussian function (black dots represent best fit). The pulse evolves into a secant hyperbolic shape as can be seen in the lower right panel by the better fit with a sech function (gray symbols) as compared with the fit of a Gaussian function (black dots). 


\subsubsection{Area theorem}

In this section we summarize the tests of our model regarding the dynamics of pulses depending on their pulse area. It had been shown by Gibbs et al. and Bullough et al. that a pulse with an area of $2 N \pi$ eventually breaks up in $N$ solitons each with an area of $2 \pi$ after propagation through the medium $[97,99]$. This can be understood as follows. Suppose that a pulse has an area of $4 \pi$, the medium then performs two Rabi cycles in which the medium, consecutively, absorbs light the first time, emits light the first time, absorbs light a second time and emits light a second time. Both emissive processes are separated in time, hence two pulses are formed. The first pulse is formed during the rising slope of the applied light pulse whereas the second pulse is formed during the falling slope of the applied light pulse. It is therefore expected that a first soliton pulse is formed with a shorter duration and a large peak amplitude (as compared to the second soliton pulse) and that a second soliton is formed with a larger duration and a lower peak amplitude. The propagation speed of the soliton pulses scales inversely with the pulse duration, therefore both pulses travel with different velocities.

We have modeled this effect by launching sech shaped pulses with several multiples of $2 \pi$ pulse area and an example is shown in fig. 6.4 for a $6 \pi$ pulse. Indeed, it can be seen that the pulse breaks-up into 3 separate solitons. Each soliton has a different maximum peak amplitude, and therefore a different propagation speed. These results are in good agreement with those as obtained by the experimental study of Gibbs et al. [99] and the numerical study by Bullough et al. [97].

\subsubsection{Colliding solitons}

An interesting property of solitons is observed upon "collision" of a faster soliton with a slower soliton within the medium as has been investigated first by Bullough et al. [97]. They showed that, in such a case, the solitons appear to be propagating through each other, experiencing a phase shift of their carrier waves, but otherwise, preserve their shape.

We have modeled such soliton collisions by launching various pairs of sech shaped $2 \pi$ pulses with different peak amplitudes into the $\Lambda$-type medium, such as to provide a propagation speed difference between them. A typical example is shown in fig. 6.5, where a slower propagating pulse is launched at $\tau_{0}=0.2 \mathrm{~ns}$ and a faster pulse is launched at $\tau_{0}=0.3 \mathrm{~ns}$. The collision of the solitons within the medium occurs at about $z=90 \mu \mathrm{m}$ and one can notice the carrier phase shifts as one soliton is slightly advanced and the other one is slightly delayed after the collision. The results we obtained with our model agree well with the results as provided by Bullough et al. [97]. 


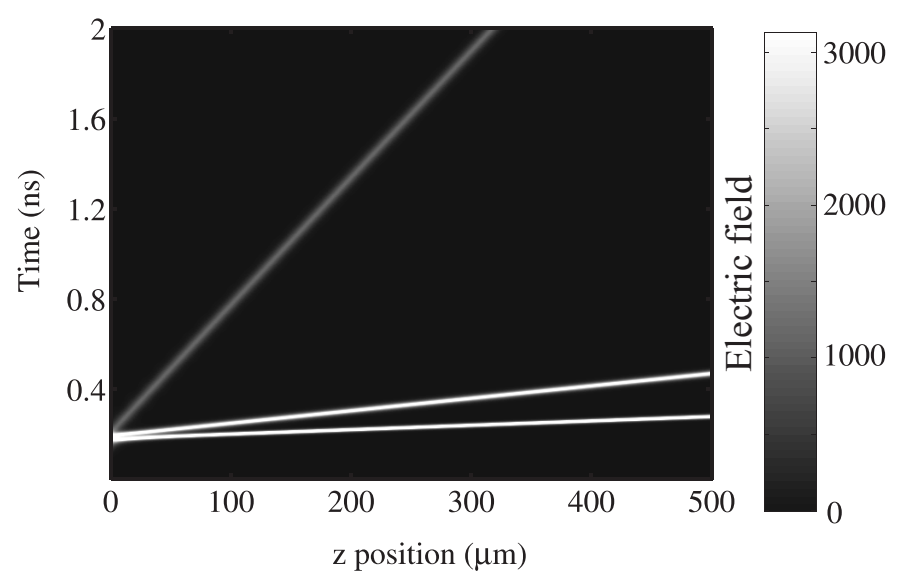

Figure 6.4: The evolution of the electric field $E_{S}$ throughout the medium. A $6 \pi$ pulse is launched into the medium at $\tau_{0}=0.2 \mathrm{~ns}$. The pulse breaks up into 3 solitons each with a different propagation speed.

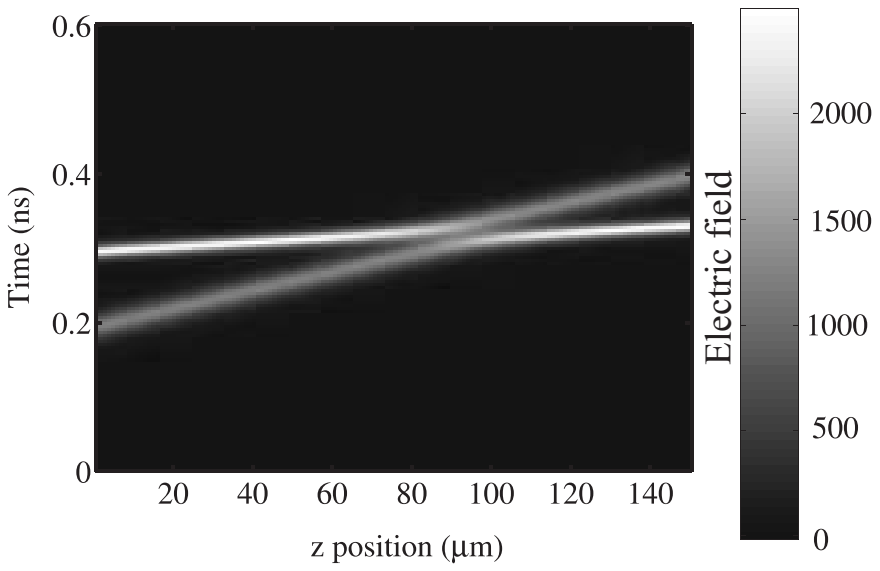

Figure 6.5: The evolution of the electric field $E_{S}$ throughout the medium after launching two solitons with different propagation speeds. The solitons cross within the medium and undergo a phase shift. 


\subsubsection{Validation of the model}

From the observation that the formation and propagation and collisions of SIT solitons, such as the examples we presented above as obtained by use of our numerical model, are in excellent agreement with those found in literature, it can be concluded that our model can be used to explore new propagation phenomena. To enable the investigation of a wide variety of phenomena beyond what is possible with two-level media, we expanded our model to the $\Lambda$-type system as was shown above (fig. 6.1) driven by two separate light fields.

\subsection{Capture and cloning event}

The $\Lambda$-type system shown in fig. 6.1 consists of three levels with two radiative transitions that connect the same excited state each to one of the complementary ground states. Note that such $\Lambda$-systems applies also to frequency degenerate transitions where the individual transitions can still be addressed, such ass via circularly polarized light [70].

In the following we show how such three-level systems allow to capture an SIT soliton by irradiating the medium with an additional light field. It will be shown that this capture removes the SIT soliton, generates a sech-shaped pulse at the complementary transition, which has been termed cloning [100], while leaving a certain spatial part of the medium in a specific ground state superposition. For demonstrating this capture and cloning scenario, we start with all of the medium in the ground state, $\rho_{11}=1$ for all $z$, in this case, when an SIT soliton with a light field $E_{S}$ enters at the $|1\rangle-|3\rangle$ transition, the coherence $\rho_{13}$ can build up proportionally with the product of the light field amplitude and the population difference between state $|1\rangle$ and $|3\rangle$, whereas the coherence $\rho_{23}$ cannot grow, since the population difference between state $|2\rangle$ and $|3\rangle$ is initially zero (both states are initially unpopulated). A pulse is launched into the medium and propagates as an SIT soliton (similar to section 6.3.1). If now a second pulse at the complementary transition with a light field described by $E_{c}$ is injected, resonant with the $|2\rangle-|3\rangle$ transition, the additional pulse does not interact with the medium wherever the $|2\rangle-|3\rangle$ transition is empty of population. However, since both transitions share the excited state, it is to be expected that the propagation of one of the light fields is influenced by the presence of the other light field. This can also be expected from the density matrix equations 2.31-2.36, where the coherence terms $\rho_{13}$ and $\rho_{23}$ both contain $\chi_{13}$ and $\chi_{23}$ terms, indicating that both light fields contribute to the dynamical behavior of these radiating terms.

The first observation of such interaction of a single SIT soliton with a control pulse was reported by Vemuri et al. [100]. They showed that a single SIT soliton on the $|1\rangle-|3\rangle$ transition in a $\Lambda$-type medium can be cloned by applying a weak control light field resonant with the $|2\rangle-|3\rangle$ transition. Further research $[41,42,47]$ showed that the spatial region of the medium, where the SIT soliton and control pulse overlap, is left with a significant population in $|2\rangle$ and a 
ground state coherence extending to either side of the region, referred to as the captured soliton. Loiko et al. and Vemuri et al. have characterized many of the important parameters influencing SIT soliton capturing, however, the phases of the light fields and the ground state coherence $\rho_{12}$, generated in such a capture event, had not been investigated. The detailed behavior of these phases has been investigated by us for the first time and will be described in section 6.5.

In order to uniquely attribute phases to the pulses and ground state coherence, we describe here a capture event, similar to the one as described by Vemuri et al. and Loiko et al. $[42,100]$. The example of the capture of an SIT soliton and the key features of this process obtained from our modeling are shown in fig. 6.6 and 6.7. In the shown example, a single SIT pulse (fig. 6.6(A)) is launched into the medium at $\tau_{0}=0.2 \mathrm{~ns}$, and initially propagates unperturbed through the medium, similar to the propagation as seen in fig. 6.2. Then a weak control pulse $E_{c}$ is launched at $\tau_{0}=0.5 \mathrm{~ns}$ and propagates with the speed of light in vacuum, which is much larger than the propagation speed of the SIT soliton (recall section 6.3.1). Therefore, the weak control pulse (too weak to be seen in fig. 6.6(B)) overtakes the SIT pulse within the medium. Unlike the collision of two solitons on the same transition these two pulses do not collide without changing the properties of the solitons. Here, as a result of the shared excited state, the control pulse is amplified at the expense of the removal (absorption) of the SIT soliton (see fig. 6.6(B)). This can be understood as follows. When the control pulse encounters the SIT soliton the control pulse finds the population in state $|3\rangle$ and zero population in $|2\rangle$, i.e., a population inversion, and the control pulse is amplified through stimulated emission. Due to the presence of both light fields, the ground state coherence $\rho_{12}$ grows and is phased such that the control light field is amplified whereas the SIT soliton light field is absorbed. As a result the energy contained in the SIT soliton light field is converted into the control light field and a part that remains in the ground state coherence ${ }^{1}$. During this conversion process, a coherence $\rho_{12}$ between the two ground states $|1\rangle-|2\rangle$ is formed at the position where the SIT soliton and control pulse overlapped during their collision. In addition, at this position also the populations of the ground states are swapped. To give a better picture of how the medium is left behind after the collision described in fig. 6.6, we have plotted the values of the ground state coherence $\left(\rho_{12}\right)$ and the associated populations of the states $|1\rangle$ and $|2\rangle$ as a function of position in the medium in fig. 6.7. As can be seen, and was confirmed by detailed inspection of many such distributions, the ground state coherence attains the shape of the first spatial derivative of a sech-pulse centered at the position of collision. What can also be seen is that the population throughout the medium remains in the initial state $\left(\rho_{11}=1\right.$ and $\left.\rho_{22}=0\right)$ except at the position of collision, where the population is swapped to exhibit a sech-shaped distribution of $\rho_{22}(z)$ and a (1-sech)-shaped distribution for $\rho_{11}(z)$.

\footnotetext{
${ }^{1}$ If the states $|1\rangle$ and $|2\rangle$ are degenerate, the soliton energy can also be fully converted into the energy of the amplified control pulse
} 
One should note, because the decay rate of the ground state coherence can be extremely low, such as in the order of seconds [95], that the shown scheme can be of potential use to store information of the involved optical pulses in the medium. Of particular interest would be to investigate, e.g., whether any phase information of the involved pulses may be stored, can be retrieved or modified as an approach for optical computing.

\subsection{Phase relations}

To prepare our discussion of optical computing in chapter 7 involving phase information storage, we introduce here a convention for the phase of a soliton pulse and the phase of a spatial distribution of coherence in the medium. We define the phase $\phi$ of the light envelope as the argument of the exponential factor in $E=A e^{i \phi} f(z, t)$ where $A$ is the real positive valued peak amplitude of the light pulse and $f$ is the real valued sech-shaped envelope. Likewise, we define the phase of the ground state coherence $\theta$ as the argument of $\rho_{12}=$ $A_{\rho} e^{i \theta} g(z)$, where $g(z)=\frac{\partial f}{\partial z}$ is the spatial shape of the coherence structure as shown in fig. 6.7.

By careful examination of the phases of the amplified control pulse and stored ground state coherence, through extensive numerical modeling of SIT soliton capture and cloning scenarios over a wide range of parameters where the phases of the SIT soliton and control light fields were varied, we found the following set of relations between the input phases and output phases:

$$
\begin{aligned}
\phi_{c, \text { out }} & =\phi_{c, \text { in }} \\
\theta & =\phi_{c}-\phi_{S}
\end{aligned}
$$

Here, $\phi_{c, \text { out }}$ is the phase of the amplified control pulse exiting the medium, $\phi_{c, i n}=\phi_{c}$ the phase of the weak control pulse which was applied to the medium, and $\phi_{S}$ the phase of the SIT soliton. These relations can be interpreted as follows. As described in the previous section, the weak control pulse is amplified at the position where it overtakes the slower SIT soliton because of the population difference between the excited state $|3\rangle$ and the ground state $|2\rangle$. It is well known from laser theory that in this process of stimulated emission, the phase of the emitted light is equal to the phase of the control light field $\phi_{c}$ [2].

The ground state coherence is left in the medium with a phase $\theta$ that is equal to the difference between the phase of the weak control pulse and the phase of the SIT pulse $\left(\phi_{S}\right)$ that were launched into the medium. Such a relation is expected upon close investigation of the right hand side of the $\dot{\rho}_{12}$ component of the density matrix equation (recall eq. 2.31) after transformation of this equation to represent the arguments (phases). This transformed equation shows the subtraction of the phases of both light fields $E_{c}$ and $E_{S}$ :

$$
\arg \left(\dot{\rho}_{12}\right)=A\left(\arg \left(E_{c}\right)-\arg \left(E_{S}\right)\right)+B\left(\arg \left(E_{c}\right)-\arg \left(E_{S}\right)\right)
$$




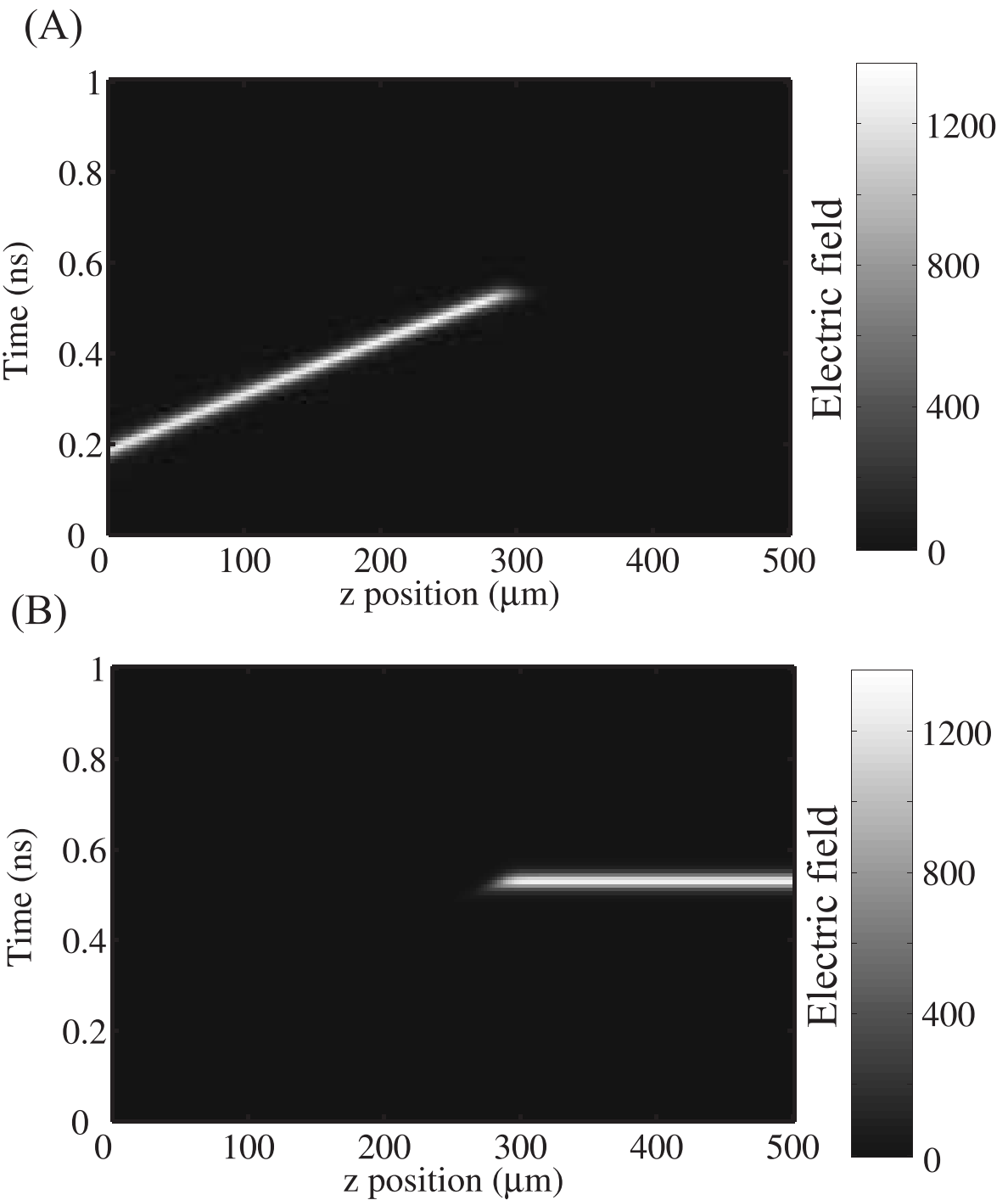

Figure 6.6: (A) Propagation of the electric field $E_{S}$ through the medium. The sech-shaped SIT pulse, launched into the medium at $\tau_{0}=0.2 \mathrm{~ns}$ on the left side in the figure, propagates initially undisturbed with a constant velocity through the medium. At $\tau_{0}=0.5 \mathrm{~ns}$ a weak control pulse is launched into the medium which overtakes the soliton after it has propagated nearly $300 \mu \mathrm{m}$ into the medium. The SIT soliton pulse is completely converted into a pulse of control light. (B) Propagation of the electric field at the control transition, $E_{c}$, through the medium. A small pulse which cannot be observed on this scale, is launched at $\tau_{0}=0.5 \mathrm{~ns}$ into the medium and overtakes an SIT soliton after propagating nearly $300 \mu \mathrm{m}$. At that position the control pulse is amplified and evolves into the shape of the SIT pulse. This pulse propagates unperturbed through the medium and exits it. 


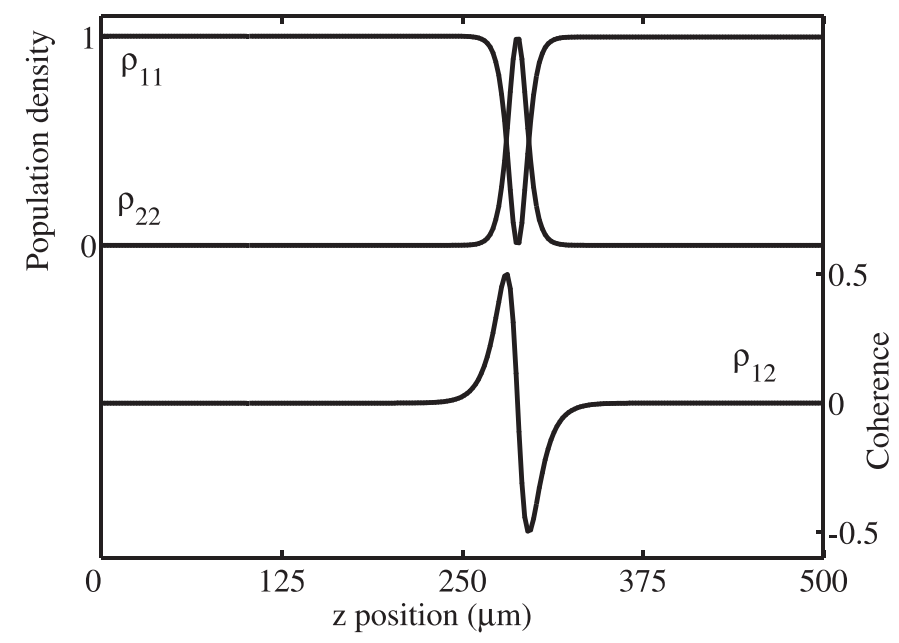

Figure 6.7: State of the nonzero density matrix elements throughout the medium after the capture event of a single SIT soliton by a weak control pulse. The excited region, where $\rho_{22}$ is non-zero, is accompanied by a region with maximal ground state coherence, $\rho_{12}$.

where $A$ and $B$ contain the resulting amplitude factors. It can be seen that, irrespective of the values of $A$ and $B$, the phase of the ground state coherence is equal to $\arg \left(E_{c}\right)-\arg \left(E_{S}\right)$ as observed by our numerical calculations.

\subsection{Conclusion}

In this chapter, a numerical model based on a coupled set of density and Maxwell's wave equations for a $\Lambda$-type medium was presented. The proper working of the model was verified via the correct display of well known features in the propagation properties of SIT solitons and their interaction with the medium under various circumstances. The validation of the model was presented by comparing the behavior of the model with the outcome of a set of theoretical and experimental results as known from literature. The model was found to be in excellent agreement.

The capture of an SIT soliton was shown to occur when a weak control pulse collides with the slower SIT soliton. In this capture event, a sech-shaped pulse at the complementary transition is formed and a spatial structure of ground state coherence is left in the medium. The relationship between the phases of inserted light pulses and the exiting pulse and the ground state coherence which remains after a soliton capture event was presented. 


\section{7 \\ Nonlinear SIT soliton propagation in $\Lambda$-type media}

\subsection{Motivation}

In chapter 6 we had recalled that nonlinear pulse propagation is involving particularly intriguing aspects if the mutual interaction of pulses and the nonlinear medium becomes strong, if the interaction is coherent due to a weak damping of coherences, and if the interaction length is long, providing large opacity. As an important example of such nonlinear pulse propagation we presented in chapter 6 the well known properties of soliton pulse propagation through opaque media, based on Self Induced Transparency (SIT). There we also reviewed the influence of an additional control light field on SIT propagation in a $\Lambda$-type medium. We suggested that such a scheme would open up opportunities to investigate the interaction and propagation of such solitons to beyond what is known so far and that such schemes might offer novel ways for optical computing.

In the following we present corresponding investigations of nonlinear interaction and propagation of SIT solitons by making use of the coupled wave and density matrix equations as described in the previous chapter. In particular, the introduction of an additional SIT soliton to the medium which had been prepared to contain a ground state coherence structure, is carefully studied and will be shown to trap SIT solitons that are injected into the medium later. The preparation of the medium with a ground state coherence, such as formed by trapping a first soliton, can have a relatively long lifetime of up to a millisecond as was shown by Liu et al. [101] (in this case using an electromagnetically induced transparency (EIT) type of process $[102,103])$. Recent work 
in both ultracold atomic gases and solids indicate that even longer lifetimes and dephasing times in the order of a second can be realized, making SIT based computational schemes, implemented in cold gases or solid state systems very interesting, to allow a larger number of computations to be performed on a single SIT soliton.

To develop an SIT-based computation scheme, we have examined the interaction of SIT solitons in a three-level system to determine what optical pulse sequences are necessary to implement logic operations. We show that after a number of solitons and control pulses have interacted with the medium, the medium possesses a ground state coherence $\left(\rho_{12}\right)$ with a spatial structure. In addition, it was found that the phase of the ground state coherence, and the phase of light emitted by the medium can be controllably changed by applying sequences of light pulses. It is observed that multiple SIT solitons can be injected into and trapped by the coherence structure of the medium within a time frame limited by the ground state coherence lifetime. In terms of computing, this implies that an SIT soliton, acting as an information carrier can be trapped in the medium and have multiple logic operations performed on it before the information is lost due to decoherence. Furthermore, we show that each operation results in a complementary pulse of light that might be used to examine the results of an operation. By examining the phase relationship between injected pulses, the coherence of the medium, and emitted pulses, it is shown that the interaction of SIT solitons are suitable as an information carrier for optical computing. To demonstrate this, a series of operations that implement an exclusive-OR (XOR) gate is presented. The XOR gate is a universal building block in quantum computing, however, it is not universal in classical computing (only NAND and NOR gates are functionally universal), therefore, further computational operations would have to be realized in further work or the applicability of the proposed scheme needs to be extended towards the domain of quantum computing operations.

To put these findings in perspective, the maximum number of operations that can be performed on a stored soliton can be estimated by comparing laser pulse repetition frequencies and ground state dephasing times. An ultracold atomic ensemble quantum memory gate with a $1 \mathrm{~ms}$ decoherence time was recently demonstrated [104], while laser repetition rates exceeding $300 \mathrm{GHz}$ have also been realized [105]. This provides an upper bound of 300 million operations on a stored SIT pulse. Even if more commonly available modelocked lasers were used, one might expect this to be on the order of 80 thousand operations.

In the following it is also shown that the ground state coherence structure, formed after a soliton capture event, can be moved through the medium by an additional control light field as was also reported by Loiko et al. [42]. This process can be used to clear stored information from the medium. Additionally, it will be shown that the ground state coherence structure can be inverted by the application of a control pulse which exceeds a threshold value. Furthermore, it will be shown that the coherence structure formed by a multiple trapping 
event can be moved and creates a bi-soliton $[106,107]$, which is a pair of two pulses that appear to be coupled during their propagation through the medium.

\subsection{Logical operations on solitons}

In all-optical computing [108], light pulses are used as carriers of information. Compared with classical computers [109], where the information is usually encoded using a binary system, in all-optical computing the information can be encoded in the presence or absence of pulses (representing bits of value 1 or 0) [110], but a much higher information capacity may be obtained, for instance, by encoding the information in the phase value of a light pulse. This phase is a so-called continuous variable [36] and may obtain any value between 0 and $2 \pi$. The information capacity can be much larger than a classical bit, provided that the resolution with which the phase can be set and measured is sufficient. As an example, the phases of ultra short pulses have been measured [111] where we estimate the phase resolution to be $0.1 \mathrm{rad}$ which corresponds to a 6 -bit capacity of a single optical pulse. And additionally, a very high control in setting the relative phases between pulses of $0.008 \pi \mathrm{rad}$ has been reported $[112,113]$.

Besides encoding information in optical pulses, it is necessary to identify methods to perform operations on the information for all-optical computing [36]. In electronic computing such operations are performed by transistors which implement logic gates to operate on the voltage that carries the information. In this section, a process which performs an XOR operation is demonstrated using optical pulses. The sequence of pulses consists of an SIT soliton pulse, which is captured by a small control pulse (such as was described in section 6.4), and, later, an interaction with a second SIT soliton pulse. The interactions between these pulses and the effect of the interactions on the phases of the stored coherence and emitted pulses are presented.

\subsubsection{Initial SIT capturing event}

In order to study the phase relations between the injected and emitted light pulses and the ground state coherence, the density matrix equations (eq. 2.31) can be written in terms of the argument (or phase) of the complex-valued amplitudes. For the equations of the phase development of the ground state coherence and the light fields $E_{c}$ and $E_{S}$ these are:

$$
\begin{aligned}
\arg \left(\dot{\rho}_{12}\right) & =A\left(\arg \left(E_{c}\right)-\arg \left(E_{S}\right)\right)+B\left(\arg \left(E_{c}\right)-\arg \left(E_{S}\right)\right) \\
\arg \left(\frac{d E_{c}}{d z}\right) & =A\left(\arg \left(E_{c}\right)+(0, \pi)\right)+B\left(\arg \left(E_{S}\right)+\arg \left(\rho_{12}\right)+\pi\right) \\
\arg \left(\frac{d E_{S}}{d z}\right) & =A\left(\arg \left(E_{S}\right)+(0, \pi)\right)+B\left(\arg \left(E_{c}\right)-\arg \left(\rho_{12}\right)+\pi\right)
\end{aligned}
$$

where $A$ and $B$ contain the resulting amplitude factors. The factor $A$ includes the amplitude of the population difference. The sign of the population 
difference sets the selected number $(0, \pi)$, where $\pi$ is chosen for stimulated absorption and 0 is chosen for stimulated emission (population inversion). By solving these differential equations the phases of the emitted light fields and the ground state coherence become equal to the phase of the derivatives $\left(\arg \left(\rho_{12}\right)=\right.$ $\left.\arg \left(\dot{\rho}_{12}\right), \arg \left(E_{c}\right)=\arg \left(\frac{d E_{c}}{d z}\right), \arg \left(E_{S}\right)=\arg \left(\frac{d E_{S}}{d z}\right)\right)$.

The initial SIT soliton capture event was described in section 6.4 , where the relations between the phases of the applied and the emitted pulses were presented. And we had shown that the phase difference between the incident soliton and the applied control field becomes stored in the medium as a ground state coherence with a particular shape located around a particular propagation coordinate. For clarity, we recall in fig. 7.1 the spatial shape of the stored ground state coherence and the associated populations. The phase of the stored ground state coherence which remains after this first capturing event is one of the components of the relationship describing the phase of the pulse induced by the second capturing event.

If the initial soliton had a phase $\phi_{S 1}$ and was captured by a weak control laser pulse with a phase $\phi_{c 1}$, then the ground state coherence $\rho_{12}$ is stored with a phase $\theta=\phi_{c 1}-\phi_{S 1}$. This capturing event can be used as an information storage process by mapping information (for instance a continuous variable phase value) onto the value of the phase difference of the two applied pulses. This phase difference is then mapped directly onto the phase of the ground state coherence.

\subsubsection{Second SIT trapping event}

We turn now to the case where a second SIT soliton is injected into the medium after the capture of a first SIT soliton. In this case, the second SIT soliton encounters an excited medium with populations and coherences shown in fig. 7.1. As will be described in more detail, it is observed that, in general, the second and also all additional SIT solitons are trapped as well upon "collision" with the excited region, and that this extends the size and modifies the spatial shape of the excited region. A distinct feature of this process is that the second and subsequent SIT solitons are trapped without a control pulse being injected. The latter is important because it allows multiple SIT soliton interactions without critically controlling the timing of their arrival in the medium.

To illustrate this, the initial capture of the first SIT soliton $\left(S_{1}\right)$ and the subsequent trapping event is described here. Fig. 7.2(a) shows the amplitude of the SIT solitons $\left(E_{S}\right)$ as a function of time and space, while fig. 7.2(b) shows the amplitude of the control light fields. An initial SIT soliton with a phase 0 (zero) is injected into the medium at time $A$, which travels slowly through the medium, as can be seen by the relatively steep slope. A weak control pulse $\left(C_{1}\right)$ with a phase of $\pi$ is injected after the SIT soliton, it is however, too weak to be visible on the scale of fig. 7.2(b) and thus represented with the dashed line. The control pulse overtakes the SIT soliton at time/position $B$, where it is amplified at the expense of the SIT soliton, producing the excited region 


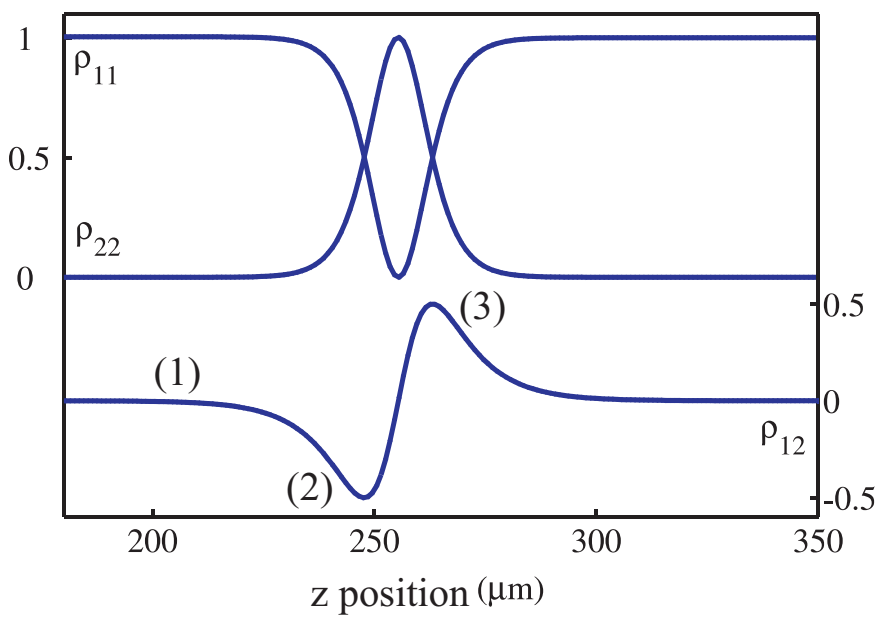

Figure 7.1: State of the nonzero density matrix elements throughout the medium after the capture (storage) of a single SIT soliton by a small control pulse. The excited region, where $\rho_{22}$ is non-zero, is accompanied by a region with maximal ground state coherence, $\rho_{12}$, which we have divided into three sections (labeled (1-3)) as an aid in the explanation of the second capture event.

illustrated in fig. 7.1. The amplified control pulse exits the medium without further interaction.

The second SIT soliton (S2), with a phase of $\pi$, is injected into the medium at time $C$, however no control light fields are injected. Nevertheless, the SIT soliton is trapped in the medium by the collision with the excited region at time/position $D$. When the SIT pulse enters the first half of the excited region at the position labeled (1) in fig. 7.1, the SIT pulse and the small amount of ground state coherence form the source for the coherence $\rho_{23}$ to grow. This coherence creates a transitory control pulse (location $E$ ) which propagates a short distance through the medium. The phase of this transitory control field $\phi_{c_{t}}$ is given by $\phi_{c_{t}}=\phi_{S 2}+\phi_{\rho_{12}}+\pi$ (recall eq. 7.2). The values of the coherence in fig. 7.1 are real valued and either positive $\left(\phi_{\rho_{12}}=0\right)$ or negative $\left(\phi_{\rho_{12}}=\pi\right.$ as is the case on the left side of the graph). Therefore, the phase of this transitory control pulse, for this particular case, is equal to the phase of the second SIT soliton.

This transitory control pulse propagates to the region labeled (2) in fig. 7.1 where, together with the ground state coherence, it forms the source term for the coherence $\rho_{13}$ to grow. This coherence creates a transitory SIT pulse (between $E$ and $F$ ) which also propagates a short distance through the medium. It was found that the phase of this SIT pulse $\phi_{S_{t}}$ is equal to the phase of the transitory control pulse (recall eq. 7.3). 

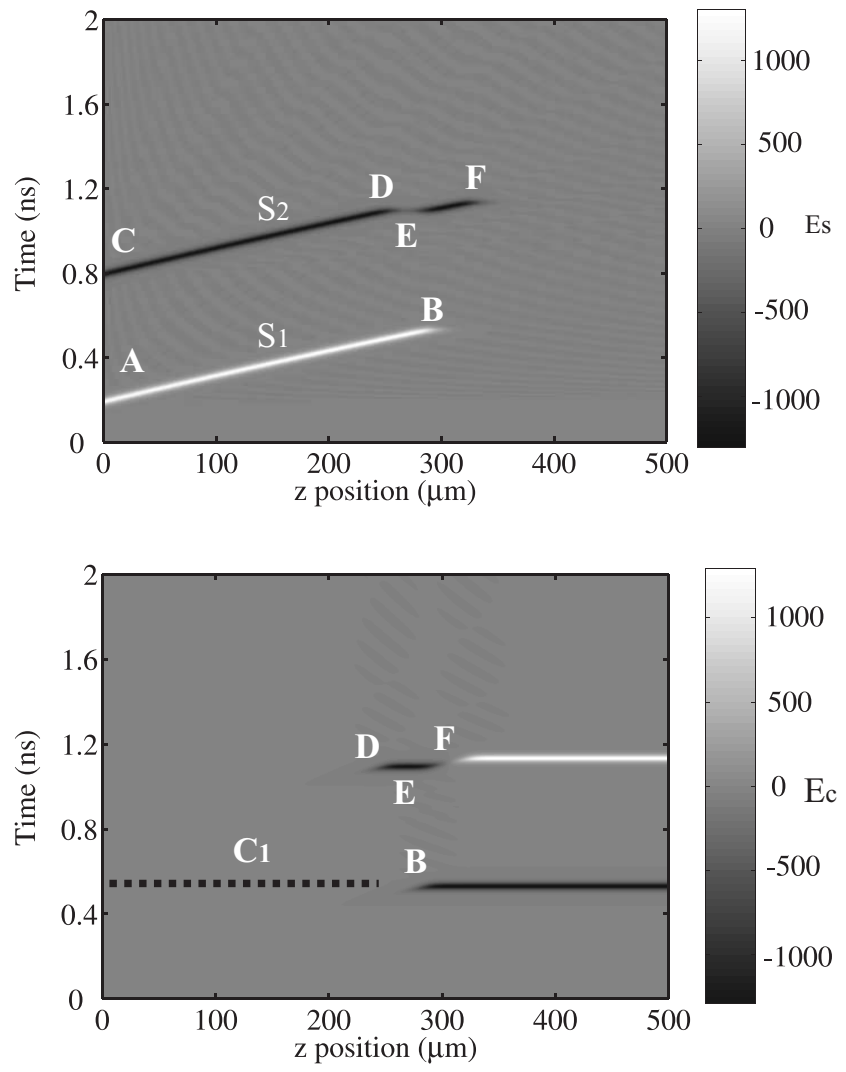

Figure 7.2: (upper) Calculated electric field amplitude of light resonant with the $|1\rangle-|3\rangle$ transition, as either an injected SIT soliton, or emission from the medium. (lower) Electric field amplitude of light resonant with the $|2\rangle-|3\rangle$ transition, as either an injected control pulse, or emission from the medium. Event $A$ : the first SIT soliton $\left(S_{1}\right)$ is introduced to the medium. Event $B$ : the SIT soliton and a weak control pulse $\left(C_{1}\right)$ overlap, resulting in the SIT soliton's capture and the control pulse's amplification. Event $C$ : after some waiting time, the second SIT soliton $\left(S_{2}\right)$ with a phase of $\pi$ is introduced to the medium. Event $D$ : the SIT soliton collides with the first half of the excited region, emitting a negative control pulse. Event $E$ : the negative control pulse captures the SIT soliton and is amplified. It then destructively interferes with the ground state coherence of the second half of the excited region, resulting in the emission of a transitory SIT soliton. Event $F$ : the remaining control light field is amplified by the transitory SIT soliton, capturing it in the process and expanding the original excitation region. 


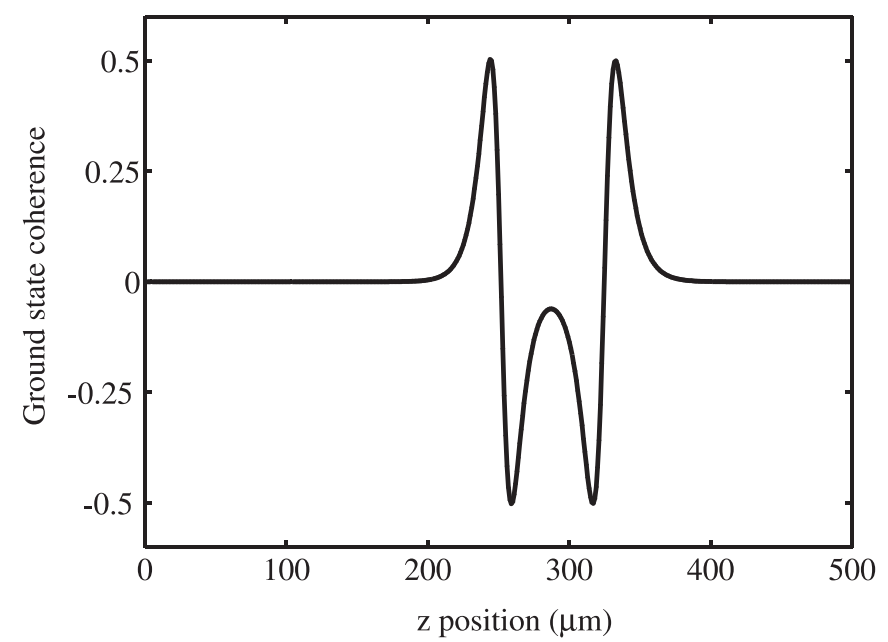

Figure 7.3: State of the ground state coherence density matrix elements throughout the medium after the trapping of a second SIT soliton. The excited region consists of two adjacent structures which were left in the medium by the first captured SIT pulse and the second trapped pulse.

The transitory SIT pulse propagates to the region labeled (3) in fig. 7.1, and creates, together with the ground state coherence, a control pulse which propagates to the end of the medium. The relation describing the phase of this control pulse was found to be $\phi_{c 2}=\phi_{S_{t}}+\pi$. This can be understood as follows: A new phase of zero is set for the ground state coherence given by the transitory SIT pulse and transitory control pulse with the same phase, using eq. 7.1 inserting this phase in eq. 7.2 we find the relation. Therefore, the phase of the control pulse that exits the medium (location $F$ ) has a phase which is rotated by an amount of $\pi$ with respect to the phase of the transitory soliton.

Besides the control pulse that exits the medium, the second SIT pulse is trapped within the medium, and as a result, the medium now has a single extended excitation region with a modified shape as is shown in fig. 7.3.

In summary, during the collision, the medium emits a control pulse, which has a phase equal to the sum of the phase of the second soliton and the phase of the stored ground state coherence as given by eq. 7.4.

$$
\phi_{c 2}=\phi_{S 2}+\phi_{c 1}-\phi_{S 1}
$$

As discussed below in section 7.2.3, it is important to note that the soliton collision results in a control pulse exiting the medium $(F)$. This is a general result, because it also occurs for subsequent collisions, as will be described in section 7.2.4. 


\subsubsection{Optical logic gates}

Here, it is shown that the continuous variable equivalent of the XOR gate can be implemented using combinations of SIT solitons and control pulses. In the continuous variable regime, using the SIT soliton phase as the information carrier, an XOR operation is given by $\left|\phi_{1}, \phi_{2}\right\rangle \rightarrow\left|\phi_{1}, \phi_{1}+\phi_{2}\right\rangle$ [36]. To implement this, the logic operation begins with the capture of the first SIT soliton (chosen with a phase $\phi_{S 1}=0$ as a reference), by the application of a control pulse $\left(\phi_{c 1}\right)$. The phase difference is transferred to the ground state coherence of the medium at the capture location, storing it $\left(\theta=\phi_{c 1}-\phi_{S 1}\right)$. Finally, the SIT soliton with the phase to be operated on $\left(\phi_{S 2}\right)$ is inserted into the medium. This SIT soliton collides with the stored coherence, generating a control pulse possessing the desired phase according to eq. $7.4\left(\phi_{c 2}=\phi_{S 2}+\phi_{c 1}\right)$.

Note that, in addition to encoding the result of the XOR operation in the phase of the emitted control pulse, the ground state coherence of the medium also stores the result of the operation (as we show in the following section). The stationary nature of the ground-state coherence then allows multiple operations to be performed. Furthermore, because each operation results in the medium emitting a pulse of light that encodes the result of that operation, conditional operations become feasible.

\subsubsection{Multiple trapping events}

After the trapping of the second soliton an extended ground state structure remains in the medium (see fig. 7.3) and acts as a trap for any subsequent SIT pulse. This is demonstrated by our calculations in which first a single SIT soliton is captured by a weak control pulse and where, for example, 10 additional SIT solitons are launched into the medium at intervals of $0.1 \mathrm{~ns}$. The results of such a calculation is seen in fig. 7.4 , where it can be seen that each of these pulses is trapped by the ground state coherence structure which expands and shifts upon each trapping event.

Each trapping event creates a control light pulse which exits the medium. The phases of each of these output pulses are given by the equation 7.4. To show that multiple operations can be performed another set of calculations was done in which a series of SIT solitons are trapped but where each of these solitons has a different phase. As an example the first ground state coherence was chosen to be stored with a phase of $0.25 \pi$ created by a suitably phased SIT soliton and weak control pulse. Subsequently, SIT pulses with a phase of $0.15 \pi, 0.35 \pi, 0.55 \pi, 0.75 \pi, 0.95 \pi, 1.15 \pi$ and $1.35 \pi$ are launched into the medium. The control pulses generated by the trapping events that exit the medium are shown in fig. 7.5 where the amplitude of the $E_{C}$-fields and the phase is shown for each pulse. As can be seen in the figure, the phases of the light fields are approximately constant over the duration of each pulse. Therefore, the phase of the output pulse can be uniquely defined as the value of the phase across such a constant region. The phases of the output pulses, indeed, show the expected values of the sum of the input SIT pulses and the 

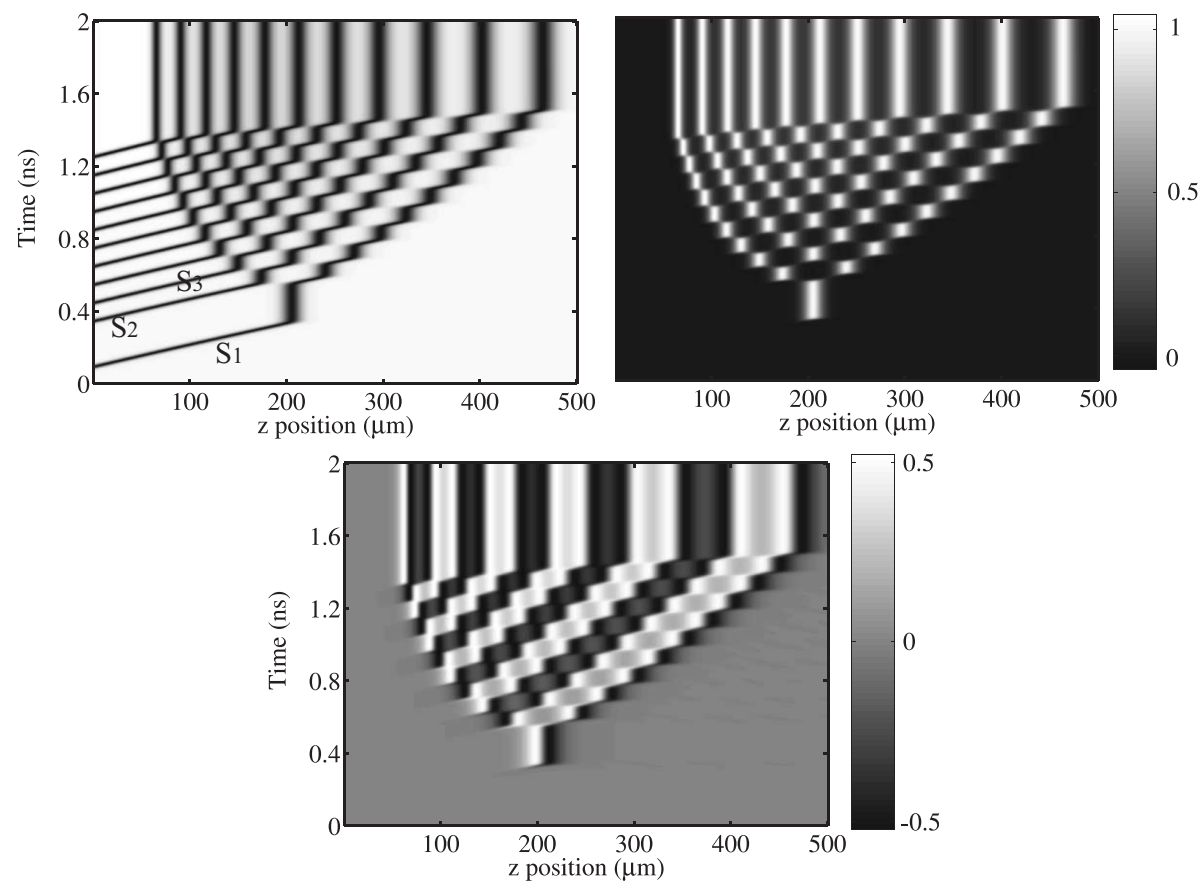

Figure 7.4: Calculated population density of $|1\rangle$ (upper left) and $|2\rangle$ (upper right) in the medium where first a single SIT soliton $\left(S_{1}\right)$ is launched at $t=0.1 \mathrm{~ns}$ and propagates until it is overtaken by a small control pulse, creating a region of stored coherence $\rho_{12}$ (lower) and switched population densities between states $|1\rangle$ and $|2\rangle$. Subsequently, 10 identical solitons $\left(S_{1}, S_{2}\right.$, etc.) are launched at intervals of $0.1 \mathrm{~ns}$, each colliding with the coherence/population structure in the medium. This collision process results in a stable structure of 11 regions of inverted ground state population. 


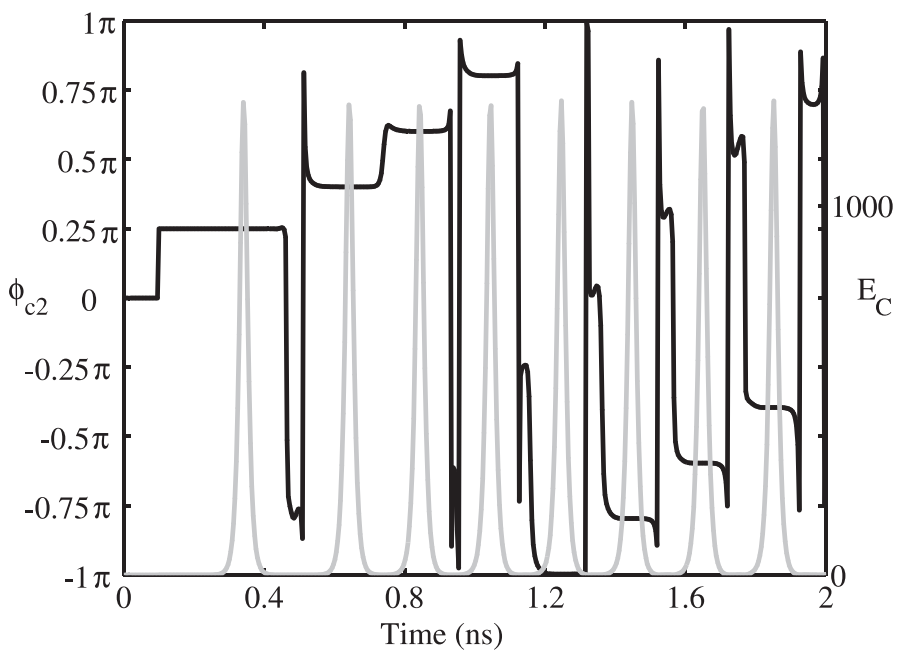

Figure 7.5: Amplitude and phase of the output control pulses as a function of time. The phase $\phi_{c 2}$ (black, on the left) of the output pulses $E_{C}$ (gray, on the right) show that the phase of the additional input SIT pulses $(0.15 \pi, 0.35 \pi, 0.55 \pi, 0.75 \pi, 0.95 \pi, 1.15 \pi, 1.35 \pi)$ is incremented with the phase of the ground state coherence $(0.25 \pi)$ each time and forms output pulses with a phase of $0.4 \pi, 0.6 \pi$, $0.8 \pi,-1 \pi,-0.8 \pi,-0.6 \pi,-0.4 \pi$ respectively. The ground state coherence was formed by a first SIT pulse with a phase 0 and a small control pulse with a phase of $0.25 \pi$ which is amplified by the trapping event and exits the medium as the first pulse with a phase of $0.25 \pi$.

stored ground state coherence phase (which is constant).

\subsection{5 $\pi$-pulse operation}

Besides the XOR gate, a different sequence of pulses acts as an XNOR gate. The output of the XNOR gate is the inversion of the output of the XOR gate, which, in the phase description, is given by $\left|\phi_{1}, \phi_{2}\right\rangle \rightarrow\left|\phi_{1}, \phi_{1}+\phi_{2}+\pi\right\rangle$ can also be seen as a $\pi$-shift with respect to the XOR operation. We discovered that such a $\pi$-shift can be induced in the system by inverting the phase of the stored ground state coherence by a sufficiently intense pulse of control light as shown in fig. 7.6. In this figure the ground state coherence is inverted by a control pulse and shifted in position.

This inversion of the sign of the ground state coherence can be understood as follows. The control pulse, incident on the excited ground state coherence region is absorbed until the population difference between states $|2\rangle$ and $|3\rangle$ 


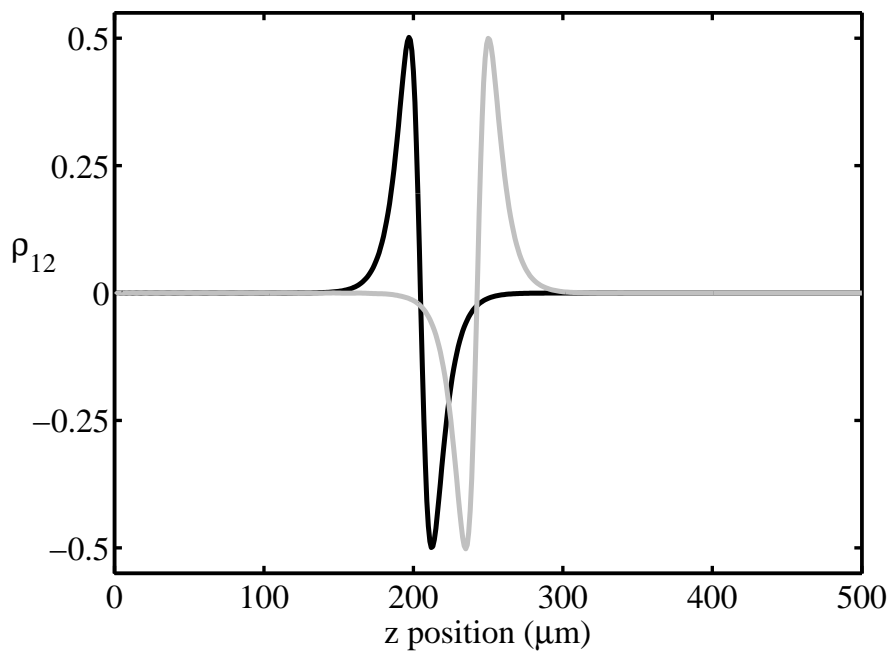

Figure 7.6: Calculated ground state coherence $\rho_{12}$ throughout the medium for the case that first a single SIT soliton is captured by a small control pulse resulting in a region of excited ground state coherence (black). Subsequently, a strong control light field pulse is applied. This pulse moves the ground state coherence structure and inverts the shape (gray), which is effectively the same as a phase addition of $\pi$.

is inverted in sign. From that point in time, the phase of the emitted control pulse, as set by the factor $A$ (recall eq. 7.2), is rotated by an amount of $\pi$. As seen in eq. 7.1, such a rotation causes the ground state coherence to rotate by an amount of $\pi$ as well. The cause of the $\pi$-shift indicates that the control pulse requires an intensity sufficient to induce a population inversion $\left(\rho_{33}>\rho_{22}\right)$, hence the observed threshold. Below this threshold value, the sign of the ground state coherence does not change as will be shown in section 7.3.

\subsection{Reading and clearing of stored ground state coherence}

Once a soliton is captured in the medium by a small control pulse, the region of swapped ground state population and excited ground state coherence remains stationary in the medium and can therefore be used for storage of information, or in computational terms act as a memory device. Now, in order to increase the functionality of such a memory device, processes that read out and clear the information need to be implemented. An indication of such a process was found in section 7.2.5, where it was shown that a control pulse shifts the position of 
the excited region in space. However, in that case, the information contained in the phase of the ground state coherence was changed, since it experienced a shift by an amount of $\pi$, which is an unwanted effect for reading out data. By use of the numerical model, it was found that such a shift does not occur when the applied control pulse does not exceed the threshold value for population inversion between state $|2\rangle$ and $|3\rangle$ to occur.

As was also pointed out by Loiko [42], the ground state coherence structure can be moved in the $+z$-direction by launching a control pulse into the medium after a soliton was captured. In this section such a calculation is repeated, where only one SIT soliton has been captured and the control pulse has an intensity below the threshold value to induce a $\pi$-shift of the ground state coherence, which is similar to the conditions as used by Loiko. These results are presented here to make the comparison to the novel dynamics, where the captured soliton has trapped a number of additional solitons and are moved by the control pulse.

To provide a typical example, the applied control pulse is chosen with a field strength that does not exceed the threshold value that would induce a $\pi$-shift of the phase of the ground state coherence. The resulting dynamical behavior of the ground state coherence $\rho_{12}$ is shown in fig. 7.7. In this figure it can be seen that the ground state coherence structure which is formed at $t=0.4 \mathrm{~ns}$ remains there until at $t=1.2 \mathrm{~ns}$ a continuous wave control field is launched into the medium, which interacts with the ground state coherence, and moves this structure in the $+z$-direction. Besides using a continuous wave control field, a single pulse (or even sets of pulses) can also be used to move the ground state coherence structure. For the case of pulses, the ground state coherence structure only moves during illumination by the control pulse and, therefore, shifts by an amount proportional to the pulse duration only.

The movement of the ground state coherence can be understood as follows. The combination of the control field and ground state coherence creates a field on the $|1\rangle-|3\rangle$ transition. This light field does not propagate as an SIT soliton but what is known as the EIT condition [27]. It designated the condition that the amplitudes of the set of light fields are such that the absorption process of each single light field is exactly counteracted by an emission process as induced by the combination of the ground state coherence and the other light field. Both fields are neither absorbed nor amplified but instead propagate together through the medium. Therefore, the excited region of ground state coherence as induced by these two light fields propagates as well.

\subsubsection{Propagation as bi-solitons}

The process of clearing a single excited region by a control light field as described in the previous section, can be applied for larger excited regions as well, as was confirmed by numerical calculations. A very interesting dynamical behavior of the propagating ground state coherence was found for a set of trapped solitons which are excited by a strong control laser field (with a value below 


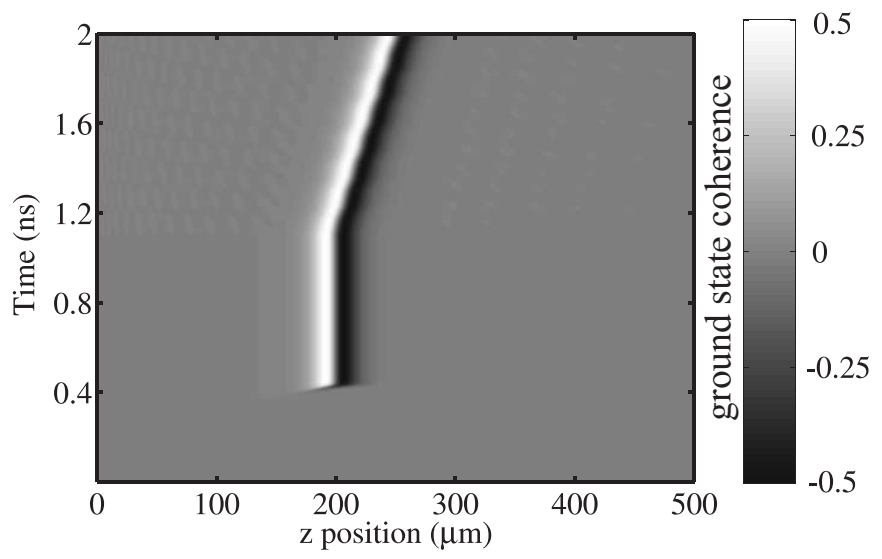

Figure 7.7: Calculated ground state coherence $\rho_{12}$ throughout the medium for a situation where first a single SIT soliton is captured by a small control pulse resulting in a region of inverted ground state population and a stable ground state coherence, here at $t=0.4 \mathrm{~ns}$ at $z \approx 200 \mu \mathrm{m}$. Later, from $t=1.2 \mathrm{~ns}$ on, a continuous wave control light field is launched into the medium. Due to the interaction of this light field with the ground state coherence structure, this structure moves in the $+z$-direction. 
the threshold needed for a $\pi$-shift of the ground state coherence to occur). In this situation, the ground state coherence structure and the additional strong control light field generate a set of SIT solitons which propagate as a larger structure through the medium (see fig. 7.8). Other systems (with different physical processes at work) in which such similar soliton structures propagate have been reported by Maruta and Hause [106, 107] where these soliton structures were named bi-solitons. However, such bi-solitons in a $\Lambda$-type system have, to our knowledge, not been reported.

The process that forms these bi-solitons can be understood as follows. The cw control light field, incident on the ground state coherence structure that was formed by the two SIT soliton pulses (one captured and one trapped), generates a pulse with a field, $E_{S}$ on resonance with the $|1\rangle-|3\rangle$ transition, at the first excited region, which propagates under EIT conditions. The $\mathrm{cw}$ control light field is not fully absorbed by this process and the remainder of the control light field propagates towards the second region of excited ground state coherence and generates a second field, $E_{S}$, which also propagates under EIT conditions. However, since the control light field at the second ground state coherence structure is weaker due to the absorption by the first ground state coherence structure, the second pulse propagates at a slower speed than the first one. Therefore, the first pulse overtakes the second pulse. This process, where one pulse overtakes the other, continues and the set of pulses propagate together during illumination with the $\mathrm{cw}$ control light field. We found that this process can be extended for multiple trapped solitons and results in the formation of combinations of solitons and bi-solitons as shown in fig. 7.9.

As can be seen in the graphs of fig. 7.9, only pairs of solitons are formed and that two of these pairs do not collide or overtake each other to form larger structures. Furthermore, for an odd number of SIT pulses, one of the pulses (the last as seen from the entrance of the medium) does not pair up. This can be understood as follows. The control field, which is partly absorbed by the first region of excited ground state coherence (the last trapped soliton), is amplified by the second region and, as a result, has approximately the same amplitude after passing excited regions (consisting of two trapped pulses) as before entering it. Furthermore, in fig. 7.5, where multiple SIT pulses were trapped in the medium, it can be seen that each new pulse creates an excited region of ground state coherence which is shorter than the previous one. The control pulse interacts with these excited regions and creates a field $E_{S}$, where the power in the pulse is proportional to the total amount of ground state coherence with which the control pulse has interacted. The propagation speed of these created pulses is proportional to the power in the pulse. Therefore, the second bi-soliton (as counted from the entrance of the medium) propagates faster than the first bi-soliton because it has a larger excited region and, as a result, generates more optical power. When an odd number of SIT pulses are inserted, the control field creates a single pulse in the $E_{S}$ field that propagates faster than the closest bi-soliton. 

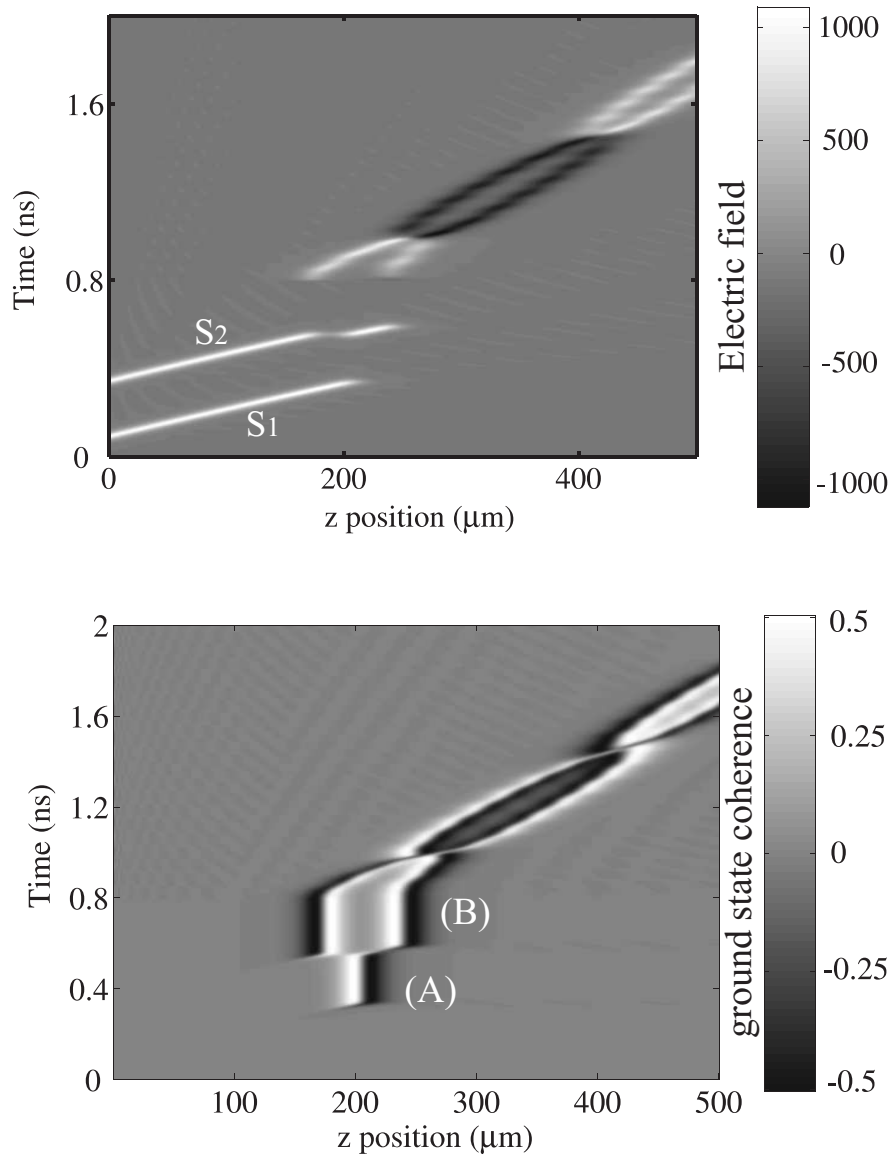

Figure 7.8: (upper) The electric field, $E_{S}$, throughout the medium for the case that first a SIT soliton $\left(S_{1}\right)$ is launched into the medium at $t=0.1 \mathrm{~ns}$ and is captured by a small control pulse. At $t=0.4 \mathrm{~ns}$ a second SIT soliton $\left(S_{2}\right)$ is launched into the medium, colliding with the ground state coherence $\rho_{12}$ structure (lower) as left over from the first SIT soliton (A), thereby creating an extended coherence structure (B). From $t=0.8 \mathrm{~ns}$ on, a continuous wave control light field with an amplitude $E_{c}=0.5 \max \left(E_{S}\right)$ is launched into the medium. This light field interacts with the extended ground state coherence structure and generates a light field $E_{S}$ which propagates as a bi-soliton. The ground state coherence moves in the positive $z$-direction. 

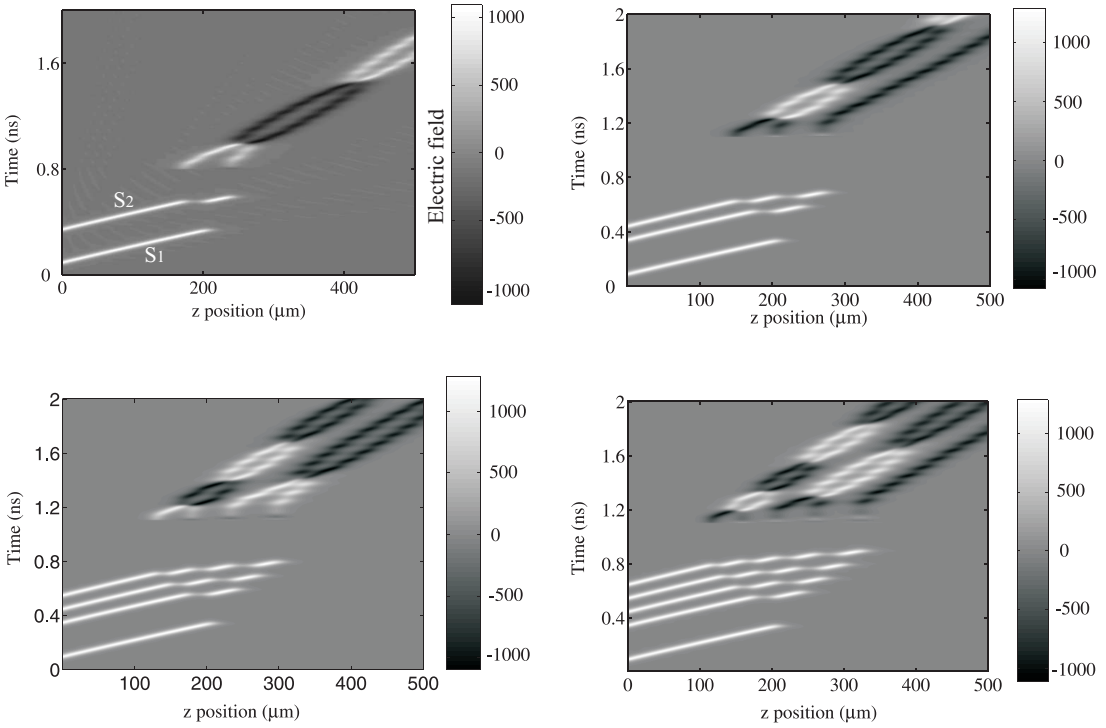

Figure 7.9: The electric field $E_{S}$ throughout the medium in a set of calculations where 1 up to 4 additional SIT solitons collide with a ground state coherence region. Subsequently from $t=1 \mathrm{~ns}$ a continuous control field is launched into the medium and interacts with the ground state coherence structure to form sets of bi-solitons and single solitons which propagate in the $+z$-direction. 


\subsection{Conclusions}

In this chapter, a highly nonlinear type of pulse propagation dynamics was investigated in a normally opaque medium. The behavior of SIT solitons in a three-level medium was analyzed by solving the density matrix and 1D Maxwell wave-equation. It was found that the phase of multiple SIT solitons can be stored as a ground state coherence in the three level medium by either the application of a control light field or by colliding the SIT soliton with a preexisting ground-state coherence. It was found that the phase of the ground state coherence, the SIT soliton and control pulses have fixed relationships, which provide a mechanism for manipulating the phase of the ground state coherence. Finally, the operations required to produce an XOR gate were demonstrated. It was noted that the results from every operation on the ground-state coherence is stored in the ground-state coherence and emitted as a control light field pulse. This provides a convenient mechanism for implementing conditional operations.

The process of clearing a medium from excited ground state regions was demonstrated. By use of an additional control light field, the ground state coherence moves through the medium until it has left it. The propagation of an excited region formed by two solitons was found to propagate as a bi-soliton. 
$\bigoplus$ 


\section{8 \\ Conclusions and discussion}

In this thesis we have presented theoretical and experimental investigations of some fundamental properties and applications of nonlinear interaction between ultrashort optical pulses and matter. A summary of the results and a discussion is presented in this chapter.

\section{SHG-based particle sizing technique of dry powders}

In an industrial process such as, e.g., the production of pharmaceutical products, a careful monitoring of the quality of the product at each different processing step is required to maximize production yield. The semi-finished products in such production processes are often powders from which the average grain size and the grain size distribution can be an important factor that determines the quality of the end-product. However, current methods to characterize powders with respect to their grainsize suffer from a set of disadvantages that makes it difficult or even impossible to use these methods for a fast, in-line characterization.

We have presented a new approach to measure the size of particles in powders via polarization angle sensitive recordings of the second harmonic generated with infrared ultrashort pulses when scanning the focused infrared beam across the powder sample. We have shown that the polarization dependent setup offers the possibility of characterizing the grain size distribution in undiluted powders. We have compared our results with measurements obtained from existing particle sizing techniques which showed a qualitative agreement considering the different measurement approach. The simplicity of our method is interesting for in-line monitoring for the food and pharmaceutical industries.

In addition, it was shown by measurements on powders of $\alpha$-lactose (monoclinic crystal class) and quartz (trigonal crystal class) that this difference in 
crystal class can be identified, by studying the shape of the function describing the SHG intensity as a function of polarization angle. This may offer the unique possibility to obtain the mixture fraction of a sample consisting of a mix of powders from different crystal classes along with the characterization of the size distribution of each of the powders.

Based on these results, it seems possible to extend SHG particle sizing towards a higher data acquisition speed which provides size distributions with higher statistical reliability, as required for applications. Such improvements could be obtained, for instance, by mounting the half-waveplate on a motorized rotation stage and by feeding the temporally changing rotation angle and SHG signal to a lock-in amplifier in order to measure the crystal axis orientation.

\section{CARS microscopy beyond the diffraction limit}

As a detailed model for another type of nonlinear interaction of ultrashort pulses with matter we described the CARS process via density matrix equations and their numerical solutions. An important advantage of this approach is that the model also includes the population distributions as well as the coherences between populations in each energy level involved. By applying this model to molecular systems consisting of a combination of electronic and vibrational states, along with driving light fields, particularly the dynamics of the population on CARS was studied. Thereby the density matrix model allowed us to draw conclusions on how to improve CARS microscopy.

In a first step, we investigated the possibility of increasing the efficiency of CARS emission by optimizing the timing between the pump, Stokes, and probe pulses. By choosing typical parameters for the properties of the molecular system it was found that the CARS signal is increased by $25 \%$ when the Stokes pulse is advanced with respect to the pump pulse by 2 ps. Similarly, delaying the probe pulse with respect to the pump pulse by 2 ps increased the efficiency. Combining these two findings, we found that the CARS efficiency can be increased beyond 30\%. These results agree qualitatively with the experimental results of Sautenkov et al., however, our approach offers additional improvements. In a standard experiment, the probe pulse is usually degenerate with the pump pulse, but we suggest to delay or to advance the probe pulse with respect to the pump pulse to increase the efficiency further. Separate pump and probe pulses with a varying mutual delay can be provided by passing a single pulse through a Michelson interferometer with an adjustable arm length or by pulse shaping techniques [114].

In a second step we have evaluated several routes to provide CARS microscopy with sub-diffraction-limited resolution for which we employed appropriate density matrix models. We have studied the interference method, as was previously described by Nikolaenko et al., and verified their claim that this scheme can suppress CARS emission. We found, however, and explain in detail that this type of interferometric suppression is not suitable for gaining sub-diffraction-limited resolution, since the relation between the amount of 
suppression and applied intensity is of linear order.

Instead, we have theoretically demonstrated novel routes to obtain subdiffraction-limited resolution in CARS microscopy. The first of these can be seen as somewhat to STED microscopy. We have shown that an intense control laser beam can be used to suppress CARS emission, when the control laser pre-populates a suitable, additional vibrational state that is non-radiatively coupled to the vibrational state that is probed by the CARS process. Such situation, in the form of neighboring vibrational states that are coupled with high transitional probabilities, can routinely be found for aqueous biological samples. Our calculations, based on typical parameters for molecular transitions used in CARS microscopy, showed that the control laser beam can yield high levels of CARS suppression, in our example up to $99.8 \%$. When employing for the CARS suppression a doughnut-shaped control laser beam with a node in its center, this would corresponds to an improved resolution of $\frac{\lambda}{22 \cdot \mathrm{NA}}$. The required intensities for the control beam, which depend on the pulse area, span a range from $200 \mathrm{MW} / \mathrm{cm}^{2}$ through to $100 \mathrm{GW} / \mathrm{cm}^{2}$, depending on the pulse duration, which seems tolerable because the molecules remain in their electronic ground state.

The implementation of a microscope based on these findings was beyond the scope of this thesis but experiments are under preparation. Regarding the choice of proper transitions, the control-level must be non-radiatively coupled to the vibrational state being probed by the CARS process, hence it is also a vibrational state. Direct excitation then requires a mid-infrared light source that is capable to deliver high peak intensities, such as optical parametric oscillators of generators followed by an optical parametric amplification.

What also would need to be taken into account is that in an experiment a much larger number of levels would be involved than assumed in our calculations, where we restricted to demonstrate the basic approach with only two vibrational levels that rapidly exchange their populations. In an experiment it might occur that pumping a single vibrational state with a control laser of narrow bandwidth is the most efficient strategy for equalizing the population between the ground state and the probed vibrational state. However, also broadband mid-infrared control lasers should be considered to excite an entire set of vibrational levels, and the best choice would depend on how well the excited levels couple to the vibrational mode that is subject to CARS imaging. If, for instance, the $\mathrm{C}-\mathrm{H}$ stretch mode of organic molecules coupled strongly to all other vibrational modes (apart from the $\mathrm{H}-\mathrm{H}$ stretch mode), it is the highest energy vibrational state, then a single fixed frequency mid-infrared laser can be added to CARS microscopy setups. However, should it be required that a different control level is found for every molecule of interest then a tunable mid-infrared laser must be used. In addition, experiments to discover the best control state would have to be performed before imaging. In place of this, it would be more desirable to use a single broad-band mid-infrared source.

Finally, the requirements for the microscope itself must be considered. First, because the control laser is a mid-infrared laser, the objective and other op- 
tics must either be reflective or use glasses suitable for wavelengths longer than $3 \mu \mathrm{m}$. Reflective objectives have a lower numerical aperture than glass objectives, while objectives made from calcium fluoride, for instance, are not commercially available. The mid-infrared laser beam must also possess a node at its center. Typically, this is achieved with a phase-plate that is optimum for a single incident wavelength. If the control laser must be tuned over a broad wavelength range, then phase-plates that perform acceptably over an equivalent wavelength range must be developed.

Regarding the optical layout of a demonstration experiment there is much similarity to a STED microscope which would be helpful for a straightforward implementation.

As an alternative approach towards sub-diffraction limited resolution in CARS microscopy, we have devised a second, novel scheme which is based on a spatially dependent amplitude modulation due to induced Rabi oscillations. Our calculations show that by resonantly driving the excitation of a control state with relatively long decoherence times, Rabi sidebands are generated in the CARS spectra. Because the sideband frequency depends on the local intensity of the control laser, by accurately measuring this Rabi splitting in the CARS spectrum, objects can be resolved to a resolution below the diffractionlimit. Using typical numbers, we have shown that a resolution in the order of $65 \mathrm{~nm}$ may be achievable. We note that our calculations also show the generation of Rabi sidebands in other molecule-specific emission lines, such as Coherent Stokes Raman Scattering. The approach towards sub-diffraction-limited resolution presented here can thus be used with this microscopy technique as well, making it more broadly applicable.

Many of the physical requirements for an experiment are the same as those discussed in the case of the saturation CARS experiment with a few important differences. The control-level is required to have a relatively long decoherence time and is to be driven with a control beam of sufficiently narrow bandwidth. Thus, a tunable narrow-band mid-infrared laser source will be required to excite a suitable control level. In comparison to the other approach we suggested (spatially selective suppression of CARS) the Rabi-labeling method is more involved regarding data recording and analysis because for each scan location of the control beam or sample an entire spectrum is to be recorded and compared to spectra from surrounding locations. The optical approach is, however, simply based on Gaussian beams and may thus be implemented into a standard microscope with little modification.

\section{SIT soliton interactions for all-optical computing}

In the last part of the thesis we theoretically investigated the propagation of ultrashort pulses in a situation where the nonlinear response of the medium is at its maximum, due to resonant excitation, and where the back-action on the light pulses is maximum as well, due to a high product of optical density and propagation length. In this situation, we predict novel phenomena to occur in 
$\Lambda$-type media, for which we have solved the coupled set of density matrix and Maxwell equations with numerical methods. We observe that soliton pulses based on self-induced transparency can be employed for interaction schemes in the context of all-optical computing. For this purpose the capture of an SIT soliton was shown to occur when a weak control pulse collides with a slower SIT soliton. In this capture event, a pulse at the complementary transition is formed and a spatial structure of ground state coherence is left in the medium. The relationship between the phases of inserted light pulses and the exiting pulse and the ground state coherence which remains after a soliton capture event was presented. This process might be used as an information storage step for all-optical computing.

Furthermore, it was found that the phase of multiple SIT solitons can be stored as a ground state coherence in the three level medium by either the application of a control light field or by colliding the SIT soliton with a preexisting ground-state coherence. It was found that the phase of the ground state coherence, the SIT soliton and control pulses have fixed relationships, which provide a mechanism for manipulating the phase of the ground state coherence. Finally, the operations required to produce an XOR gate were demonstrated. It was noted that the results from every operation on the ground-state coherence is stored in the ground-state coherence and emitted as a control light field pulse. This provides a convenient mechanism for implementing conditional operations.

Finally, the process of clearing a medium from excited ground state regions was demonstrated, which can be seen as a reset of the medium. By use of an additional control light field, the ground state coherence moves through the medium in a controlled manner until it has left it. The propagation of an excited region formed by two solitons was found to propagate as a bi-soliton.

We propose that an experimental demonstration of the nonlinear interaction processes, such as capturing and subsequent trapping of SIT solitons, may be realized using a cooled Pr:YSO crystal, for instance. This crystal possesses a $\Lambda$ type system with the required long lived ground state population lifetime of 100 seconds and an excited state lifetime of $111 \mu \mathrm{s}$ which is long enough to enable SIT interaction using standard laser systems [95]. Furthermore, the detuning of the lasers due to Doppler shifts are minimized because of the absence of atomic motion within the crystal, which is required for the phase relations as described in chapter 7 to hold. The properties of the crystal should therefore suffice to experimentally demonstrate the theoretically predicted SIT soliton interaction leading to the capture of an SIT soliton and the observation of the complementary cloned pulse, and, in addition, the trapping of additional SIT solitons and the XOR gate operation within such a medium as proposed in chapter 7 . 
$\oplus_{\oplus}$ 


\section{Bibliography}

[1] T. H. Maiman, "Optical and Microwave-Optical Experiments in Ruby," Physical Review Letters 4, 564 (1960).

[2] M. Sargent III, M. O. Scully, and W. E. Lamb jr., Laser physics (AddisonWesley Publishing Company, 1974).

[3] C. Tang and L. Cheng, Fundamentals of optical parametric processes and oscillators (Harwood academic publishers, 1995).

[4] O. Svelto, Principles of lasers (Springer, 1998).

[5] N. Laitinen, O. Antikainen, and J. Yliruusi, "Does a powder surface contain all necessary information for particle size distribution analysis?" European Journal of Pharmaceutical Sciences 17, 217-227 (2002).

[6] H. Larhrib, X. M. Zeng, G. P. Martin, C. Marriott, and J. Pritchard, "The use of different grades of lactose as a carrier for aerosolised salbutamol sulphate," International Journal of Pharmaceutics 191, 1-14 (1999).

[7] B. Berne and R. Pecora, "Dynamic Light Scattering," (John Wiley \& Sons, New York, 1976).

[8] J. Cornillault, "Particle Size Analyzer," Applied Optics 11, 265-265 (1972).

[9] N. Laitinen, Opening New Perspectives for Visual Characterisation of Pharmaceutical Solids, Ph.D. thesis, University of Helsinki (2003).

[10] J. Malik, S. Belongie, T. Leung, and J. Shi, "Contour and Texture Analysis for Image Segmentation," International Journal of Computer Vision 43, 7-27 (2001).

[11] E. Abbe, "Beiträge zur Theorie des Mikroskops und der mikroskopischen Wahrnehmung," Archiv für Mikroskopische Anatomie 9, 413-468 (1873).

[12] S. W. Hell and J. Wichmann, "Breaking the diffraction resolution limit by stimulated emission: stimulated-emission-depletion fluorescence microscopy," Optics Letters 19, 780-782 (1994). 
[13] T. A. Klar, S. Jakobs, M. Dyba, A. Egner, and S. W. Hell, "Fluorescence microscopy with diffraction resolution barrier broken by stimulated emission," Proceedings of the National Academy of Sciences of the United States of America 97, 8206-8210 (2000).

[14] E. Betzig, G. H. Patterson, R. Sougrat, O. W. Lindwasser, S. Olenych, J. S. Bonifacino, M. W. Davidson, J. Lippincott-Schwartz, and H. F. Hess, "Imaging Intracellular Fluorescent Proteins at Nanometer Resolution," Science 313, 1642-1645 (2006).

[15] M. Rust, M. Bates, and X. Zhuang, "Sub-diffraction-limit imaging by stochastic optical reconstruction microscopy (STORM)," Nature Methods 3, 793-796 (2006).

[16] B. Huang, S. A. Jones, B. Brandenburg, and X. Zhuang, "Whole-cell 3D STORM reveals interactions between cellular structures with nanometerscale resolution," Nature Methods 5, 1047-1052 (2008).

[17] E. Rittweger, K. Y. Han, S. E. Irvine, C. Eggeling, and S. W. Hell, "STED microscopy reveals crystal colour centres with nanometric resolution," Nature Photonics 3, 144-147 (2009).

[18] M. Dyba and S. W. Hell, "Focal Spots of Size $\lambda / 23$ Open Up Far-Field Florescence Microscopy at $33 \mathrm{~nm}$ Axial Resolution," Physical Review Letters 88, 163901 (2002).

[19] R. Zenobi, "Analytical tools for the nano world," Analytical and Bioanalytical Chemistry 390, 215-221 (2008).

[20] H. Ikagawa, M. Yoneda, M. Iwaki, Z. Isogai, K. Tsujii, R. Yamazaki, T. Kamiya, and M. Zako, "Chemical Toxicity of Indocyanine Green Damages Retinal Pigment Epithelium," Investigative Ophthalmology Visual Science 46, 2531-2539 (2005).

[21] H. H. Szeto, P. W. Schiller, K. Zhao, and G. Luo, "Fluorescent dyes alter intracellular targeting and function of cell-penetrating tetrapeptides," The FASEB Journal pp. 04-1982fje (2004).

[22] J. W. Dobrucki, D. Feret, and A. Noatynska, "Scattering of Exciting Light by Live Cells in Fluorescence Confocal Imaging: Phototoxic Effects and Relevance for FRAP Studies," Biophysical Journal 93, 1778-1786 (2007).

[23] C. J. Daly and J. C. McGrath, "Fluorescent ligands, antibodies, and proteins for the study of receptors," Pharmacology \& Therapeutics $\mathbf{1 0 0}$, 101-118 (2003).

[24] H. Heinzelmann and D. W. Pohl, "Scanning near-field optical microscopy," Applied Physics A: Materials Science \& Processing 59, 89-101 (1994). 
[25] H. G. Frey, S. Witt, K. Felderer, and R. Guckenberger, "High-Resolution Imaging of Single Fluorescent Molecules with the Optical Near-Field of a Metal Tip," Physical Review Letters 93, 200801 (2004).

[26] L. V. Hau, S. E. Harris, Z. Dutton, and C. H. Behroozi, "Light speed reduction to 17 metres per second in an ultracold atomic gas," Nature 397, 594-598 (1999).

[27] S. E. Harris, "Electromagnetically Induced Transparency," Physics Today 50, 36-42 (1997).

[28] G. Huang, K. Jiang, M. G. Payne, and L. Deng, "Formation and propagation of coupled ultraslow optical soliton pairs in a cold three-state double- $\Lambda$ system," Physical Review E 73, 056606 (2006).

[29] A. Kuzmich, W. P. Bowen, A. D. Boozer, A. Boca, C. W. Chou, L. M. Duan, and H. J. Kimble, "Generation of nonclassical photon pairs for scalable quantum communication with atomic ensembles," Nature $\mathbf{4 2 3}$, 731-734 (2003).

[30] N. Gisin and R. Thew, "Quantum communication," Nature Photonics 1, $165-171$ (2007).

[31] J. H. Plantenberg, P. C. de Groot, C. J. P. M. Harmans, and J. E. Mooij, "Demonstration of controlled-NOT quantum gates on a pair of superconducting quantum bits," Nature 447, 836-839 (2007).

[32] M. Shuker, O. Firstenberg, R. Pugatch, A. Ron, and N. Davidson, "Storing Images in Warm Atomic Vapor," Physical Review Letters 100, $223601-4$ (2008).

[33] D. G. Cory, A. F. Fahmy, and T. F. Havel, "Ensemble quantum computing by NMR spectroscopy," Proceedings of the National Academy of Sciences of the United States of America 94, 1634-1639 (1997).

[34] N. A. Gershenfeld and I. L. Chuang, "Bulk Spin-Resonance Quantum Computation," Science 275, 350-356 (1997).

[35] S. L. McCall and E. L. Hahn, "Self-Induced Transparency by Pulsed Coherent Light," Physical Review Letters 18, 908 (1967).

[36] S. Lloyd and S. L. Braunstein, "Quantum Computation over Continuous Variables," Physical Review Letters 82, 1784 (1999).

[37] I. L. Chuang and Y. Yamamoto, "Simple quantum computer," Physical Review A 52, 3489 (1995).

[38] K. Nemoto and W. J. Munro, "Nearly Deterministic Linear Optical Controlled-NOT Gate," Physical Review Letters 93, 250502 (2004). 
[39] M. A. Duguay and J. W. Hansen, "An ultrafast light gate," Applied Physics Letters 15, 192-194 (1969).

[40] N. Matsuda, Y. Mitsumori, H. Kosaka, K. Edamatsu, and R. Shimizu, "Lossless all-optical phase gate using a polarization-division Sagnac interferometer applicable to a waveguide-type Kerr medium," Applied Physics Letters 91, 171 119-3 (2007).

[41] W. P. Beeker, D. N. Deriga, and K. J. Boller, "Capture of solitons in layered phaseonium," in Quantum Electronics Conference, 2005. International 1, 718-719 (2005).

[42] Y. Loiko, C. Serrat, R. Vilaseca, V. Ahufinger, J. Mompart, and R. Corbalan, "Ultrashort pulse control of space-dependent excitations in a three-level system," Physical Review A (Atomic, Molecular, and Optical Physics) 75, 023 801-9 (2007).

[43] R. W. Boyd, Nonlinear optics (Academic press, San Diego, 2003).

[44] P. W. Milonni and J. H. Eberly, Lasers (John Wiley \& Sons, New York, 1988).

[45] V. G. Dmitriev, Handbook of Nonlinear Optical Crystals (Springer, 1999).

[46] R. J. Roberts, R. C. Rowe, and P. York, "The relationship between indentation hardness of organic solids and their molecular structure," Journal of Materials Science 29, 2289-2296 (1994).

[47] Y. Loiko and C. Serrat, "Coherent and phase-sensitive phenomena of ultrashort laser pulses propagating in three-level Lambda-type systems studied with the finite-difference time-domain method," Physical Review A (Atomic, Molecular, and Optical Physics) 73, 063 809-11 (2006).

[48] J. Butcher, Numerical methods for ordinary differential equations (Wiley, 2003).

[49] C. B. Rawle, C. J. Lee, C. J. Strachan, K. Payne, P. J. Manson, and T. Rades, "Towards characterization and identification of solid state pharmaceutical mixtures through second harmonic generation," Journal of Pharmaceutical Sciences 95, 761-768 (2006).

[50] R. Hellwarth and P. Christensen, "Nonlinear optical microscopic examination of structure in polycrystalline ZnSe," Optics Communications 12, 318-322 (1974).

[51] R. Hellwarth and P. Christensen, "Nonlinear Optical Microscope Using Second Harmonic Generation," Applied Optics 14, 247-248 (1975).

[52] N. Bloembergen and P. S. Pershan, "Light Waves at the Boundary of Nonlinear Media," Physical Review 128, 606 (1962). 
[53] Y. R. Shen, "Optical Second Harmonic Generation at Interfaces," Annual Review of Physical Chemistry 40, 327-350 (2003).

[54] J. N. Gannaway and C. J. R. Sheppard, "Second-harmonic imaging in the scanning optical microscope," Optical and Quantum Electronics 10, 435-439 (1978).

[55] Y. Uesu, S. Kurimura, and Y. Yamamoto, "Optical second harmonic images of $90^{\circ}$ domain structure in $\mathrm{BaTiO}_{3}$ and periodically inverted antiparallel domains in $\mathrm{LiTaO}_{3}$, " Applied Physics Letters 66, 2165-2167 (1995).

[56] E. D. Mishina, N. E. Sherstyuk, K. A. Vorotilov, A. S. Sigov, R. Barberi, M. P. Moret, F. Manders, M. P. De Santo, P. K. Larsen, and T. Rasing, "Nonlinear optical and electrostatic force microscopy for ferroelectric polarization imaging," Applied Physics B: Lasers and Optics 74, 783-788 (2002).

[57] Y. Guo, P. P. Ho, H. Savage, D. Harris, P. Sacks, S. Schantz, F. Liu, N. Zhadin, and R. R. Alfano, "Second-harmonic tomography of tissues," Optics Letters 22, 1323-1325 (1997).

[58] W.-L. Chen, T.-H. Li, P.-J. Su, C.-K. Chou, P. T. Fwu, S.-J. Lin, D. Kim, P. T. C. So, and C.-Y. Dong, "Second harmonic generation chi tensor microscopy for tissue imaging," Applied Physics Letters 94, 183902-3 (2009).

[59] R. Gauderon, P. B. Lukins, and C. J. R. Sheppard, "Three-dimensional second-harmonic generation imaging with femtosecond laser pulses," Optics Letters 23, 1209-1211 (1998).

[60] Y. Nakata, G. Soumagne, T. Okada, and M. Maeda, "Pulsed-laser deposition of barium titanate films and plume dynamics," Applied Surface Science 127-129, 650-654 (1998).

[61] A. Savoia, D. Paparo, P. Perna, Z. Ristic, M. Salluzzo, F. M. Granozio, U. S. di Uccio, C. Richter, S. Thiel, J. Mannhart, and L. Marrucci, "Polar catastrophe and electronic reconstructions at the $\mathrm{LaAlO}_{3} / \mathrm{SrTiO}_{3}$ interface: Evidence from optical second harmonic generation," Physical Review B (Condensed Matter and Materials Physics) 80, 075 110-6 (2009).

[62] M. Sliwa, K. Nakatani, T. Asahi, P. G. Lacroix, R. B. Pansu, and H. Masuhara, "Polarization and wavelength dependent nonlinear optical properties of a photo-switchable organic crystal," Chemical Physics Letters 437, 212-217 (2007).

[63] P. Stoller, B.-M. Kim, A. M. Rubenchik, K. M. Reiser, and L. B. Da Silva, "Polarization-dependent optical second-harmonic imaging of a rat-tail tendon," Journal of Biomedical Optics 7, 205-214 (2002). 
[64] G. Binnig, C. F. Quate, and C. Gerber, "Atomic Force Microscope," Physical Review Letters 56, 930 (1986).

[65] D. McMullan, "Scanning electron microscopy 1928-1965," Scanning 17, 175-185 (1995).

[66] M. Minsky, "Microscopy apparatus," (1961).

[67] B. J. Albers, M. Liebmann, T. C. Schwendemann, M. Z. Baykara, M. Heyde, M. Salmeron, E. I. Altman, and U. D. Schwarz, "Combined low-temperature scanning tunneling/atomic force microscope for atomic resolution imaging and site-specific force spectroscopy," Review of Scientific Instruments 79, 033 704-9 (2008).

[68] V. Van Ngo, M. Hernandez, B. Roth, and D. C. Joy, "STEM Imaging of Lattice Fringes and beyond in a UHR In-Lens Field-Emission SEM," Microscopy Today 15, 12-16 (2007).

[69] D. Takamatsu, Y. Yamakoshi, and K.-i. Fukui, "Photoswitching Behavior of a Novel Single Molecular Tip for Noncontact Atomic Force Microscopy Designed for Chemical Identification," The Journal of Physical Chemistry B 110, 1968-1970 (2006).

[70] T. L. Meek, An introduction to spectroscopy, atomic structure and chemical bonding (Canoe Press, 1998).

[71] M. A. Yuratich and D. C. Hanna, "Coherent anti-Stokes Raman spectroscopy (CARS) - Selection rules, depolarization ratios and rotational structure," Molecular Physics: An International Journal at the Interface Between Chemistry and Physics 33, 671 - 682 (1977).

[72] C. Serrat, "Coherent control of ultrafast optical four-wave mixing with two-color $\omega-3 \omega$ laser pulses," Physical Review A 72, 023808 (2005).

[73] J.-X. Cheng, A. Volkmer, and X. S. Xie, "Theoretical and experimental characterization of coherent anti-Stokes Raman scattering microscopy," Journal of the Optical Society of America B 19, 1363-1375 (2002).

[74] K. A. Stankov and V. P. Tzolov, "Nonlinear mirror based on raman interactions," Applied Physics B: Lasers and Optics 52, 96-101 (1991).

[75] H. Wang, Y. Fu, and J.-X. Cheng, "Experimental observation and theoretical analysis of Raman resonance-enhanced photodamage in coherent anti-Stokes Raman scattering microscopy," Journal of the Optical Society of America B 24, 544-552 (2007).

[76] R. de Vivie-Riedle and U. Troppmann, "Femtosecond Lasers for Quantum Information Technology," Chemical Reviews 107, 5082-5100 (2007). 
[77] J. B. Asbury, T. Steinel, C. Stromberg, K. J. Gaffney, I. R. Piletic, A. Goun, and M. D. Fayer, "Hydrogen Bond Dynamics Probed with Ultrafast Infrared Heterodyne-Detected Multidimensional Vibrational Stimulated Echoes," Physical Review Letters 91, 237402 (2003).

[78] A. J. Wurzer, T. Wilhelm, J. Piel, and E. Riedle, "Comprehensive measurement of the S1 azulene relaxation dynamics and observation of vibrational wavepacket motion," Chemical Physics Letters 299, 296-302 (1999).

[79] W. M. Tolles, J. W. Nibler, J. R. McDonald, and A. B. Harvey, "A Review of the Theory and Application of Coherent Anti-Stokes Raman Spectroscopy (CARS)," Applied Spectroscopy 31, 253-271 (1977).

[80] S. Postma, A. C. W. van Rhijn, J. P. Korterik, P. Gross, J. L. Herek, and H. L. Offerhaus, "Application of spectral phase shaping to high resolution CARS spectroscopy," Optics Express 16, 7985-7996 (2008).

[81] M. Jurna, J. P. Korterik, C. Otto, J. L. Herek, and H. L. Offerhaus, "Background free CARS imaging by phase sensitive heterodyne CARS," Optics Express 16, 15 863-15869 (2008).

[82] V. A. Sautenkov, C. Y. Ye, Y. V. Rostovtsev, G. R. Welch, and M. O. Scully, "Enhancement of field generation via maximal atomic coherence prepared by fast adiabatic passage in Rb vapor," Physical Review A 70, 033406 (2004).

[83] J. Oreg, F. T. Hioe, and J. H. Eberly, "Adiabatic following in multilevel systems," Physical Review A 29, 690 (1984).

[84] U. Gaubatz, P. Rudecki, M. Becker, S. Schiemann, M. Külz, and K. Bergmann, "Population switching between vibrational levels in molecular beams," Chemical Physics Letters 149, 463-468 (1988).

[85] A. Nikolaenko, V. V. Krishnamachari, and E. O. Potma, "Interferometric switching of coherent anti-Stokes Raman scattering signals in microscopy," Physical Review A (Atomic, Molecular, and Optical Physics) 79, 013 823-7 (2009).

[86] J. A. Armstrong, N. Bloembergen, J. Ducuing, and P. S. Pershan, "Interactions between Light Waves in a Nonlinear Dielectric," Physical Review 127, 1918 (1962).

[87] V. Westphal and S. W. Hell, "Nanoscale Resolution in the Focal Plane of an Optical Microscope," Physical Review Letters 94, 143903 (2005).

[88] R. F. Begley, A. B. Harvey, and R. L. Byer, "Coherent anti-Stokes Raman spectroscopy," Applied Physics Letters 25, 387-390 (1974). 
[89] H. Okamoto and K. Yoshihara, "Femtosecond time-resolved coherent Raman scattering from $\beta$-carotene in solution. Ultrahigh frequency $(11 \mathrm{THz})$ beating phenomenon and sub-picosecond vibrational relaxation," Chemical Physics Letters 177, 568-572 (1991).

[90] B. Hein, K. I. Willig, and S. W. Hell, "Stimulated emission depletion (STED) nanoscopy of a fluorescent protein-labeled organelle inside a living cell," Proceedings of the National Academy of Sciences 105, 14271 14276 (2008).

[91] C. Ventalon, J. M. Fraser, M. H. Vos, A. Alexandrou, J.-L. Martin, and M. Joffre, "Coherent vibrational climbing in carboxyhemoglobin," Proceedings of the National Academy of Sciences of the United States of America 101, 13216-13220 (2004).

[92] T. Witte, J. S. Yeston, M. Motzkus, E. J. Heilweil, and K. L. Kompa, "Femtosecond infrared coherent excitation of liquid phase vibrational population distributions (v>5)," Chemical Physics Letters 392, 156-161 (2004).

[93] P. Schouwink, H. v. Berlepsch, L. Dähne, and R. F. Mahrt, "Dependence of Rabi-splitting on the spatial position of the optically active layer in organic microcavities in the strong coupling regime," Chemical Physics 285, 113-120 (2002).

[94] V. A. Sautenkov, Y. V. Rostovtsev, and E. R. Eliel, "Observation of narrow Autler-Townes components in the resonant response of a dense atomic gas," Physical Review A (Atomic, Molecular, and Optical Physics) 78, 013 802-5 (2008).

[95] A. V. Turukhin, V. S. Sudarshanam, M. S. Shahriar, J. A. Musser, B. S. Ham, and P. R. Hemmer, "Observation of Ultraslow and Stored Light Pulses in a Solid," Physical Review Letters 88, 023602 (2001).

[96] R. C. Hilborn, "Einstein coefficients, cross sections, f values, dipole moments, and all that," American Journal of Physics 50, 982-986 (1982).

[97] R. K. Bullough, P. J. Caudrey, J. C. Eilbeck, and J. D. Gibbon, "A general theory of self-induced transparency," Optical and Quantum Electronics 6, 121-140 (1974).

[98] R. F. Gutterres, C. Amiot, A. Fioretti, C. Gabbanini, M. Mazzoni, and O. Dulieu, "Determination of the ${ }^{87} \mathrm{Rb} 5 \mathrm{p}$ state dipole matrix element and radiative lifetime from the photoassociation spectroscopy of the $R_{b_{2}}$ $0_{g}{ }^{-}\left(\mathrm{P}_{3 / 2}\right)$ long-range state," Physical Review A 66, 024502 (2002).

[99] H. M. Gibbs and R. E. Slusher, "Sharp-Line Self-Induced Transparency," Physical Review A 6, 2326 (1972). 
[100] G. Vemuri, G. S. Agarwal, and K. V. Vasavada, "Cloning, Dragging, and Parametric Amplification of Solitons in a Coherently Driven, Nonabsorbing System," Physical Review Letters 79, 3889 (1997).

[101] C. Liu, Z. Dutton, C. H. Behroozi, and L. V. Hau, "Observation of coherent optical information storage in an atomic medium using halted light pulses," Nature 409, 490-493 (2001).

[102] K. J. Boller, A. Imamoglu, and S. E. Harris, "Observation of electromagnetically induced transparency," Physical Review Letters 66, 2593 (1991).

[103] D. F. Phillips, A. Fleischhauer, A. Mair, R. L. Walsworth, and M. D. Lukin, "Storage of Light in Atomic Vapor," Physical Review Letters 86, 783 (2001).

[104] B. Zhao, Y.-A. Chen, X.-H. Bao, T. Strassel, C.-S. Chuu, X.-M. Jin, J. Schmiedmayer, Z.-S. Yuan, S. Chen, and J.-W. Pan, "A millisecond quantum memory for scalable quantum networks," Nature Physics 5, 95-99 (2009).

[105] K. Merghem, A. Akrout, A. Martinez, G. Aubin, A. Ramdane, F. Lelarge, and G. H. Duan, "Pulse generation at $346 \mathrm{GHz}$ using a passively mode locked quantum-dash-based laser at $1.55 \mu \mathrm{m}$," Applied Physics Letters 94, 021 107-3 (2009).

[106] A. Maruta, T. Inoue, Y. Nonaka, and Y. Yoshika, "Bisoliton propagating in dispersion-managed system and its application to high-speed and longhaul optical transmission," IEEE Journal of Selected Topics in Quantum Electronics 8, 640-650 (2002).

[107] A. Hause, H. Hartwig, M. Bohm, and F. Mitschke, "Binding mechanism of temporal soliton molecules," Physical Review A (Atomic, Molecular, and Optical Physics) 78, 063 817-9 (2008).

[108] A. McAulay, Optical computer architectures : the application of optical concepts to next generation computers (Wiley, New York, 1991).

[109] A. Turing, "On computable numbers, with an application to the entscheidungsproblem," Proceedings of the London Mathematical Society 42, 230-265 (1936).

[110] Y.-D. Wu, T.-T. Shih, and M.-H. Chen, "New all-optical logic gates based on the local nonlinear Mach-Zehnder interferometer," Optics Express 16, 248-257 (2008).

[111] P. Bowlan, P. Gabolde, A. Shreenath, K. McGresham, R. Trebino, and S. Akturk, "Crossed-beam spectral interferometry: a simple, highspectral-resolution method for completely characterizing complex ultrashort pulses in real time," Optics Express 14, 11892-11900 (2006). 
[112] S.-H. Shim, D. B. Strasfeld, and M. T. Zanni, "Generation and characterization of phase and amplitude shaped femtosecond mid-IR pulses," Optics Express 14, 13120-13130 (2006).

[113] P. Tian, D. Keusters, Y. Suzaki, and W. S. Warren, "Femtosecond Phase-Coherent Two-Dimensional Spectroscopy," Science 300, 15531555 (2003).

[114] A. M. Weiner, "Femtosecond pulse shaping using spatial light modulators," Review of Scientific Instruments 71, 1929-1960 (2000). 


\section{Dankwoord}

Hoewel alleen mijn naam op de voorkant van dit proefschrift prijkt, is de inhoud ervan tot stand gekomen door de bijdrage van een groot aantal mensen, die, soms direkt, soms indirekt, hebben meegeholpen bij het tot stand komen van dit werk. Een zeer belangrijke bijdrage is geleverd door Chris Lee en Klaus Boller. Met hen heb ik in de afgelopen jaren een aantal verschillende onderzoeksthema's op het gebied van niet-lineaire optica bestudeerd, waarvan we de drie meest succesvolle richtingen in dit proefschrift hebben opgetekend. De onderlinge discussies, waarin vele creatieve, nieuwe ideëen werden geopperd, konden weliswaar lang duren, maar hebben er uiteindelijk toe geleid dat er resultaten werden geboekt waaruit een aantal mooie publicaties en conferentiebijdragen zijn voortgekomen.

I would also like to thank the members of my promotion committee for the time and effort they spent in studying my thesis. The results, which were obtained through a cooperation with the groups of Carsten Fallnich and Jennifer Herek, have formed a solid basis for this thesis. Ook wil ik Allard Mosk bedanken voor zijn interesse gedurende de afgelopen jaren in de vorderingen van mijn onderzoek. En een speciaal woord van dank aan Dave Blank bij wie ik een tijdlang in de vakgroep heb gewerkt op zoek naar studieonderwerpen op het raakvlak van niet-lineaire optica en materiaalkunde.

Door het werken binnen twee vakgroepen zijn er natuurlijk ook vele anderen die door discussies of het geven van presentaties zowel inspiratie hebben gegeven voor mijn onderzoek, alsook feed-back over het werk. Met het gevaar, per ongeluk, een naam te vergeten, tracht ik hierbij toch een lijst te geven, omdat ik weet dat jullie je naam hier tussen zullen zoeken. Dank aan: Rolf, Arie, Thijs, Arjen, Arjen, Joska, Josée, Andreas, Ab, Mark, Thomas, Peter, Ruud, Robert, Fred, Piet, Bert, Leon, Simone, Gerard, Jacob, Arsen, Guus, Gertjan, Mark, André, Bernhard, Marion, Henk, Sjoerd, José, Gerrit, Matthijn, Ole, Evert, Hajo, Peter, Ruud, Fran(s)(k), Hans, Jeroen, Antony, Gerwin, Michiel, Mercy, Sajid, Tomek, Oktay, Xin, Wolter, Paul (wie is dat?), Vittorio en iedereen die hier dus per ongeluk niet tussen staat.

Naast werken worden er ook weleens koffiepauzes gehouden, en bij LPNO worden die altijd van discussievoer (dat wil zeggen $80 \%$ over auto's) voorzien door de heren van het NCLR: Arco, Cees, Ramon, Leon, Gerald, Hein en Liviu (ook nog bedankt voor de tijd binnen LPNO). Ook jullie bedankt voor deze afleiding, maar ook voor de zeker zinvolle discussies.

In het vorige studiejaar heb ik een groep eerstejaars studenten begeleid met 
hun opdracht voor het vak OTNW. Met hen, Ale, Bas, Chiel en Thijs, zijn de eerste experimenten verricht die uiteindelijk hebben geleid tot de meetopstelling voor de SHG scan metingen. Hen wil ik graag bedanken voor hun interesse in het vakgebied waardoor deze onderzoeksrichting uiteindelijk tot een paar mooie resultaten heeft geleid.

Mijn studievereniging Arago dient ook bedankt te worden, al was het maar voor de interne streeplijst waarop ik vele genoten candybars achter mijn naam kon optekenen. De organisatie van de activiteiten en borrels van de afgelopen jaren lag in handen van vele besturen en commissies en hen wil ik daarom bedanken voor het werk dat daarmee gedaan werd.

Nog een vereniging die zeker niet vergeten mag worden, is het Musica Silvestra Orkest, waarbij ik, als trotse tweede violist, al sinds het begin van mijn studietijd meespeel. De talrijke reizen, repetitieweekenden en andere activiteiten van het orkest waren stuk voor stuk mooie evenementen, maar ook de wekelijkse repetitie en de bijbehorende derde helft is een welkome afwisseling van de werkweek door de grote diversiteit in persoonlijkheden van mijn mede musici. Aan alle MSO'ers en oud-MSO'ers dus een woord van dank. Een zeer inspannende week heb ik beleefd met Wouter, Anne en Mario door ook nog even mee te doen met de vierdaagse van Nijmegen, het leert je in ieder geval wat doorzettingsvermogen is!

Nog een club, inmiddels zelfs vereniging, waar ik nog regelmatig goede discussies mocht voeren is UReka, dé studenten-URaadspartij van de UT. De meedenkersbijeenkomsten en meedenkers-weekenden, maar natuurlijk ook de borrel-discussie-avonden waren niet alleen zeer gezellig, maar helpen ook in de bekwaming van de kunst van het verdedigen van stellingen. Dus alle URekanen, bedankt voor de mooie discussies!

Komen we aan bij de paragraaf: Bier. Bedankt! Vooral natuurlijk aan mijn mede-bierbrouwers van de Brak, met wie ik talloze prachtige activiteiten heb meegemaakt die qua uiterlijk niet direct aan bier doen denken, zoals de ski-reizen en de zeil-weekenden, maar die daar uiteindelijk wel van doordrenkt waren. Uiteraard draait het natuurlijk echt om de mooie zaken die we met elkaar beleven en kunnen bespreken, dus dank aan alle heeren van de Bracque.

Mijn broertje en zusje wil ik ook graag bedanken, niet alleen omdat ze als paranimf mij bij mijn promotie ondersteunen, maar ook voor hun steun in de afgelopen jaren. Daarvoor wil ik natuurlijk ook mijn ouders en opa en oma bedanken, die, ook al wisten ze dat ze slechts weinig zouden begrijpen van de inhoud van mijn werk, toch steeds geïnteresseerd bleven vragen naar wat de nieuwste ontwikkelingen waren.

De afsluiting van dit proefschrift is, noodzakelijkerwijs, ook het begin van "de tijd na je promotie" en ik kan mij geen betere manier indenken dan die samen met Josée tegemoet te gaan. Dank je voor je continue steun. 